\title{
Giesekus Constitutive Model for Thermoviscoelastic Fluids based on Ordered Rate Constitutive Theories
}

\author{
K. S. Surana ${ }^{1, *}$, D. Nunez ${ }^{1}$ and J. N. Reddy ${ }^{2}$ \\ ${ }^{1}$ Mechanical Engineering, University of Kansas, Lawrence, Kansas, USA \\ ${ }^{2}$ Mechanical Engineering, Texas A\&M University, College Station, Texas, USA
}

\begin{abstract}
This paper presents derivation of Giesekus constitutive model in Eulerian description based on ordered rate constitutive theories for thermoviscoelastic fluids for compressible and incompressible cases in contra-, co-variant and Jaumann bases. The ordered rate constitutive theories for thermoviscoelastic fluids of orders $(m, n)$ consider convected time derivative of order $m$ of the deviatoric Cauchy stress tensor in a chosen basis (i.e. co-, contra-variant or Jaumann) as dependent variable in the development of constitutive theories for the stress tensor. Its argument tensors consist of density, temperature, convected time derivatives of the deviatoric Cauchy stress tensor of up to order $m-1$ and convected time derivative of up to order $n$ of the conjugate strain tensor. In addition, constitutive theory for the heat vector compatible with the constitutive theory for the deviatoric stress tensor is also presented in co-, contra-variant and Jaumann bases. It is shown that the Giesekus constitutive model is a subset of the rate constitutive theory of orders $m=n=1$. It is also shown that the deviatoric Cauchy stress tensor (contra-, co-variant or Jaumann basis) naturally results as dependent variable in the constitutive theory, and that currently used Giesekus constitutive model in deviatoric polymer Cauchy stress tensor is not derivable based on axioms and principles of the constitutive theory in continuum mechanics. Numerical studies are presented for fully developed flow between parallel plates for a dense polymeric liquid using the Giesekus constitutive model derived in this paper as well as currently used model.
\end{abstract}

Keywords: Contravariant, covariant, Jaumann, upper convective, lower convective, least squares

\section{INTRODUCTION}

The polymeric fluids can be classified in two broad categories: dilute polymeric fluids and dense polymeric fluids or polymer melts. Compressibility in polymeric fluids is only important at very high pressures. Generally, polymeric fluids are treated as incompressible, hence it is appropriate to say polymeric liquids. Dilute polymeric fluids are primarily much like Newtonian fluids but with some elastic effects, i.e. behavior is dominated by viscous effects. In such fluids, solvent viscosity is dominant, i.e. much higher than polymer viscosity. Polymer melts are dense polymeric fluids whose behavior is dominated by elastic effects. In such fluids the polymer viscosity is much higher than the solvent viscosity. Polymeric fluids are of significant industrial importance. The first attempt to derive constitutive equations for polymers appears to have been due to Maxwell [1]. Later these were generalized to remove the small displacement assumption [2,3]. Maxwell constitutive model is a linear viscoelastic model. Using Maxwell model as a basis, the Jeffreys model is obtained by adding time derivative of the symmetric part of the velocity gradient tensor [2]. Generalization of the Maxwell model is obtained by superposition of a series of Maxwell models [2,3].

It is commonly accepted [2] that linear viscoelastic models have many limitations: (1) They can not describe shear rate dependent viscosity (2) They can not describe normal stress behavior accurately (3) They

*Address corresponding to this author at the Department of Mechanical Engineering, University of Kansas, Lawrence, KS, USA; Tel: 785864-2988; E-mail: kssurana@ku.edu fail to describe small-strain phenomena if it is accompanied by large displacements due to rigid rotations. These lead to the development of 'Quasi-linear differential models'. The Oldroyd-B [4] model falls into this category. Deficiencies of these models in describing realistic physical flow phenomena in polymer melts lead to the development of non-linear differential constitutive models for polymeric fluids. Giesekus model [5] and PTT model [6] fall into this category. Many other constitutive models have been proposed for polymeric fluids (see reference [2]). The fundamental driving principles in the development of these models have been anisotropic drag due to Brownian motion of polymer molecules and their networks and the kinetic theory $[2,3]$.

First, we remark that polymeric fluids at a macro scale are viewed as isotropic homogeneous continuous media. Thus, in our view, the constitutive theory for such fluids must be derivable using principles and axioms of continuum mechanics. In fact, Maxwell constitutive model has been derived in reference [7] using the theory of generators and invariants. It is instructive to examine the derivations of Giesekus constitutive model based on continuum mechanics axioms and principles and as a subset of ordered polymeric fluids as it may suggest new possibilities for improvement in the existing models. The thermoviscoelastic fluids have memory and hence exhibit relaxation phenomenon. It can be shown [2] that for fluids with relaxation phenomenon, we must at the very least consider the first convected time derivative of the deviatoric Cauchy stress tensor as a dependent variable in the development of the constitutive theory. Additionally, the deviatoric Cauchy stress tensor and the 
first convected time derivative of the conjugate strain tensor must be considered as its argument tensors (in addition to others). The ordered rate constitutive theories presented in references $[8,9]$ are generalizations of this concept. When a polymer is subjected to disturbance, the motion of the polymer molecules is complex (Brownian motion [10,11]). The origin of the development of the currently used Giesekus constitutive model seems to be based on Brownian motion and kinetic theory $[2,3]$ in which the polymer deviatoric Cauchy stress is used as a dependent variable in the constitutive theory. In the work presented in this paper, we view dense polymers (described by Giesekus model) as homogeneous, isotropic continuous media within the continuum mechanics framework. The derivation of the constitutive theories is initiated using the second law of thermodynamics (entropy inequality). The conditions resulting from the entropy inequality are further utilized in conjunction with the theory of generators and invariants (see section 5) in the development of general ordered rate constitutive theories. It is shown that Giesekus constitutive model is derivable from the ordered rate constitutive theory for thermoviscoelastic fluids in which $m=1$ and $n=1$, i.e. the rate constitutive theory of order 1 in deviatoric stress and strain measures. This approach based on entropy inequality ensures that when this constitutive model is used in conjunction with other conservation laws, thermodynamic equilibrium of the deforming polymeric fluid is ensured. It is shown that this approach necessitates that we consider deviatoric Cauchy stress tensor (in contra- or co-variant basis or Jaumann) as dependent variable in the development of the constitutive theory as opposed to deviatoric Cauchy stress based on polymer stress as used currently.

The paper presents derivations of the constitutive theory for the stress tensor for the Giesekus constitutive model as well as the compatible constitutive theory for the heat vector. The derivations are presented in contravariant as well as covariant bases resulting in upper convected and lower convected rate constitutive theories. Additionally, the constitutive theories are also presented using Jaumann rates. Numerical studies are presented for fully developed flow between parallel plates for a dense polymeric liquid using the rate constitutive model presented in this paper as well as the currently used Giesekus constitutive model.

\section{CONSIDERATIONS IN THE DEVELOPMENT OF THE GIESEKUS CONSTITUTIVE MODEL}

For any deforming matter to be in thermodynamic equilibrium, the conservation laws (conservation of mass, balance of momenta, conservation of energy and the second law of thermodynamics) must be satisfied. Since, balance of momenta and conservation of en- ergy assume existence of a stress field and heat vector without specific regard to the constitution of the matter, conservation of mass, balance of momenta and conservation of energy are applicable to all deforming matter. Thus, these three conservation laws do not provide a basis for the development of the constitutive theory for any deforming matter. Hence, the second law of thermodynamics (or entropy inequality) must be considered in the development of the constitutive theory. When the mathematical model of deforming matter is derived using conservation of mass, balance of momenta and conservation of energy, and the constitutive theory is derived using the second law of thermodynamics, the thermodynamic equilibrium of the deforming matter is ensured.

For thermoviscoelastic fluids, Eulerian description is necessary for the development of the mathematical models due to the fact that complex motion material points in such fluids may be difficult to describe using Lagrangian description, thus the constitutive theories for such fluids must also be developed in Eulerian description. In Eulerian description we monitor the state of the deforming matter at a fixed location in the current configuration, hence the material point displacements are not known and therefore the use of strain measures in the development of the constitutive theories yields constitutive equations in which strain measures are not defined. Thus, in Eulerian description of motion, we must consider strain rates in the development of the constitutive theories, hence the name 'rate constitutive theories'. In the following we present notations, definitions and various measures of the stresses, finite strains and their convected time derivatives that are essential in the development of rate constitutive theories for ordered thermoviscoelastic fluids. The rate constitutive theory of order 1 in both stress and strain measures forms the basis for deriving the Giesekus constitutive model for the stress tensor and the compatible constitutive theory for the heat vector.

\subsection{Notation}

In this section we present a brief account of coordinate systems, measures of stresses, strain and their convected time derivatives of various orders, and helpful definitions introduced in [9] but repeated here for convenience. Let $x_{i}$ and $\bar{x}_{i}$ denote position coordinates of a material point in the reference and current configurations, respectively, in a fixed frame ( $x$-frame)

$$
\begin{aligned}
& \bar{x}_{i}=\bar{x}_{i}\left(x_{1}, x_{2}, x_{3}, t\right) \\
& x_{i}=x_{i}\left(\bar{x}_{1}, \bar{x}_{2}, \bar{x}_{3}, t\right)
\end{aligned}
$$

If $\{d x\}=\left[d x_{1}, d x_{2}, d x_{3}\right]^{T}$ and $\{d \bar{x}\}=\left[d \bar{x}_{1}, d \bar{x}_{2}, d \bar{x}_{3}\right]^{T}$ are the components of length $d s$ and $d \bar{s}$ in the reference and current configurations, and if we neglect the 
infinitesimals of orders two and higher in both configurations, then we obtain

$$
\begin{aligned}
& \{d \bar{x}\}=[J]\{d x\} \\
& \{d x\}=[\bar{J}]\{d \bar{x}\}
\end{aligned}
$$

with

$$
[J]=[\bar{J}]^{-1} ;[\bar{J}]=[J]^{-1} ;[J][\bar{J}]=[\bar{J}][J]=[I]
$$

and using Murnaghan's notation

$$
[J]=\left[\frac{\bar{x}_{1}, \bar{x}_{2}, \bar{x}_{3}}{x_{1}, x_{2}, x_{3}}\right] ;[\bar{J}]=\left[\frac{x_{1}, x_{2}, x_{3}}{\bar{x}_{1}, \bar{x}_{2}, \bar{x}_{3}}\right]
$$

in which the columns of $[J]$ are covariant base vectors $\tilde{\boldsymbol{g}}_{i}$ whereas the rows of $[\bar{J}]$ are contravariant base vectors $\tilde{\boldsymbol{g}}^{i}$. $[J]$ and $[\bar{J}]$ are Jacobians of deformation. The basis of $[\bar{J}]$ is reciprocal of the basis of $[J]$. The following relations are useful in the sequel:

$$
\begin{aligned}
& \frac{D}{D t}[J]=[\bar{L}][J] \\
& \frac{D}{D t}[\bar{J}]=-[\bar{J}][\bar{L}] \\
& \bar{L}_{i j}=\frac{\partial \bar{v}_{i}}{\partial \bar{x}_{j}}
\end{aligned}
$$

in which $\frac{D}{D t}$ stands for the material time derivative, $[\bar{L}]$ is the spatial velocity gradient tensor and $\bar{v}_{i}$ are velocity components of a material point $\bar{x}_{i}$ in the current configuration in the $x$-frame.

\subsection{Strain Measures}

In the present work we consider Green's strain tensor $[\varepsilon]$, in covariant basis, and Almansi strain tensor $[\bar{\varepsilon}]$, in contravariant basis, both are measures of finite strain and have Cartesian dyads in the $x$-frame

$$
\begin{array}{ll}
{[\varepsilon]=\frac{1}{2}\left([J]^{T}[J]-[I]\right)} & \text { (Def.) } \\
{[\bar{\varepsilon}]=\frac{1}{2}\left([I]-[\bar{J}][\bar{J}]^{T}\right)} & \text { (Def.) }
\end{array}
$$

The term $[\bar{J}][\bar{J}]^{T}$ in (2.11) is the replacement for the usual terms $[\bar{J}]^{T}[\bar{J}]$ that occurs in the derivation of $[\bar{\varepsilon}]$. This is necessary to ensure that $[\varepsilon]$ and $[\bar{\varepsilon}]$ have the same dyads in the $x$-frame. This difference arises due to the fact that columns of $[J]$ are covariant base vectors whereas contravariant base vectors are rows of $[\bar{J}]$. Thus in the expression for $[\bar{\varepsilon}]=\frac{1}{2}\left([I]-[\bar{J}]^{T}[\bar{J}]\right),[\bar{J}]^{T}$ must be replaced with $[\bar{J}]$ and likewise, $[\bar{J}]$ must take the place of $[\bar{J}]^{T}$ so that $[\varepsilon]$ and $[\bar{\varepsilon}]$ have the same dyads [9].

\subsection{Stress Measures}

Let $\left[\bar{T}^{(0)}\right]$ be the contravariant Cauchy stress tensor (derived using contravariant basis) that corresponds to the directions normal to the faces of the deformed tetrahedron in the current configuration. Let $\left[\bar{T}_{(0)}\right]$ be the covariant Cauchy stress tensor (derived using covariant basis) that corresponds to a new tetrahedron such that covariant base vectors are normal to its faces.

The stress tensors $\left[\bar{T}^{(0)}\right]$ and $\left[\bar{T}_{(0)}\right]$ are the Eulerian measures as these correspond to the deformed tetrahedron in the current configuration. When considering the development of mathematical models or constitutive theory in the Lagrangian description, it is essential to obtain the Lagrangian stress measures that correspond to $\left[\bar{T}^{(0)}\right]$ and $\left[\bar{T}_{(0)}\right]$ stress tensors. Using $\left[\bar{T}^{(0)}\right]$, areas of the faces of the deformed tetrahedron and a correspondence rule, we can derive the second Piola-Kirchhoff stress tensor $\left[T^{[0]}\right]$ corresponding to the contravariant Cauchy stress tensor $\left[\bar{T}^{(0)}\right]$ whose components act on the faces of the undeformed tetrahedron in the reference configuration. We refer to $\left[T^{[0]}\right]$ as the second PiolaKirchhoff stress tensor based on contravariant Cauchy stress tensor $\left[\bar{T}^{(0)}\right]$. Similarly, if we use $\left[\bar{T}_{(0)}\right]$, areas of the faces of a new tetrahedron (obtained from the true deformed tetrahedron such that the covariant base vectors are normal to its faces) and a new correspondence rule, we can also derive another second PiolaKirchhoff stress tensor $\left[T_{[0]}\right]$ whose components also act on the faces of the undeformed tetrahedron in the reference configuration. We refer to $\left[T_{[0]}\right]$ as the second Piola-Kirchhoff stress tensor based on covariant Cauchy stress tensor $\left[\bar{T}_{(0)}\right]$. Following reference [9] we can write (for a compressible matter)

$$
\begin{aligned}
& {\left[T^{[0]}\right]=\left[T^{[0]}\right]^{T}=|J|[\bar{J}]\left[\bar{T}^{(0)}\right][\bar{J}]^{T} \quad \text { (Def.) }} \\
& {\left[T_{[0]}\right]=\left[T_{[0]}\right]^{T}=|J|[J]^{T}\left[\bar{T}_{(0)}\right][J] \quad \text { (Def.) }}
\end{aligned}
$$

The Jaumann stress tensor $\left[{ }^{(0)} \bar{T}^{J}\right]$ is defined as average of the contravariant and covariant Cauchy stress tensors with the further restriction of being equal to the contravariant and covariant Cauchy stress tensors. Thus

$$
\left[{ }^{(0)} \bar{T}^{J}\right]=\left[\bar{T}^{(0)}\right]=\left[\bar{T}_{(0)}\right] \quad \text { (Def.) }
$$

and hence

$$
\left[{ }^{(0)} \bar{T}^{J}\right]=\frac{1}{2}\left(\left[\bar{T}^{(0)}\right]+\left[\bar{T}_{(0)}\right]\right)
$$

We note that $\left[\bar{T}^{(0)}\right]=\left[\bar{T}_{(0)}\right]$ only holds for infinitesimal deformation in which contra- and co-variant Cauchy stress tensors are identical. If the deformation deviates from infinitesimal assumption, then $\left[\bar{T}^{(0)}\right] \neq\left[\bar{T}_{(0)}\right]$ and hence the Jaumann stress measure becomes spurious. $\left.{ }^{[0)} \bar{T}^{J}\right]$ stress measures has been used often in the published work specially for solid matter and in many instances for finite deformation studies [12,13], hence the reason for including this stress measures in the present work and subsequently the rate constitutive theory developments based on this measure of stress. 


\subsection{Convected Time Derivatives of Green's and Al- mansi Strain Tensors and Jaumann Strain Rates}

Following references $[7,9]$ and noting that Green's strain tensor is a covariant measure whereas the Almansi strain tensor is a contravariant measure, both for finite strain, we can summarize their convected time derivatives in co- and contra-variant bases. These hold for both compressible as well as incompressible matter.

\subsubsection{Covariant Basis: Convected Time Derivatives of Green's Strain Tensor}

If $\left[\gamma_{(j)}\right] ; j=1,2, \ldots, n$ are the convected time derivatives of $[\varepsilon]$ of orders $j=1,2, \ldots, n$ in covariant basis and if $\left[\gamma_{[j]}\right] ; j=1,2, \ldots, n$ are the material derivatives of orders $j=1,2, \ldots, n$ of $[\varepsilon]$, then we can write

$$
\begin{aligned}
& {\left[\gamma_{[k]}\right]=\frac{D}{D t}\left[\gamma_{[k-1]}\right]=[J]^{T}\left[\gamma_{(k)}\right][J]} \\
& {\left[\gamma_{(k)}\right]=\frac{D}{D t}\left[\gamma_{(k-1)}\right]+[\bar{L}]^{T}\left[\gamma_{(k-1)}\right]+\left[\gamma_{(k-1)}\right][\bar{L}]}
\end{aligned}
$$

with $k=2,3, \ldots$ and

$$
\begin{aligned}
& {\left[\gamma_{[1]}\right]=\frac{D}{D t}[\varepsilon]=[J]^{T}\left[\gamma_{(1)}\right][J]} \\
& {\left[\gamma_{(1)}\right]=\frac{1}{2}\left([\bar{L}]+[\bar{L}]^{T}\right)=[\bar{D}]}
\end{aligned}
$$

where $\left[\gamma_{(j)}\right] ; j=1,2, \ldots, n$ are fundamental kinematic tensors in the covariant basis. It is straight forward to show that these are objective.

\subsubsection{Contravariant Basis: Convected Time Deriva- tives of Almansi Strain Tensor}

If $\left[\gamma^{(j)}\right] ; j=1,2, \ldots, n$ are the convected time derivatives of $[\bar{\varepsilon}]$ of orders $j=1,2, \ldots, n$ in contravariant basis and if $\left[\gamma^{[j]}\right] ; j=1,2, \ldots, n$ are the material derivatives of orders $j=1,2, \ldots, n$ of $[\bar{\varepsilon}]$, then we can write

$$
\begin{aligned}
& {\left[\gamma^{[k]}\right]=\frac{D}{D t}\left[\gamma^{[k-1]}\right]=[\bar{J}]\left[\gamma^{(k)}\right][\bar{J}]^{T}} \\
& {\left[\gamma^{(k)}\right]=\frac{D}{D t}\left[\gamma^{(k-1)}\right]-[\bar{L}]\left[\gamma^{(k-1)}\right]-\left[\gamma^{(k-1)}\right][\bar{L}]^{T}}
\end{aligned}
$$

with $k=2,3, \ldots$ and

$$
\begin{aligned}
& {\left[\gamma^{[1]}\right]=\frac{D}{D t}[\bar{\varepsilon}]=[\bar{J}]\left[\gamma^{(1)}\right][\bar{J}]^{T}} \\
& {\left[\gamma^{(1)}\right]=\frac{1}{2}\left([\bar{L}]+[\bar{L}]^{T}\right)=[\bar{D}]}
\end{aligned}
$$

where $\left[\gamma^{(j)}\right] ; j=1,2, \ldots, n$ are fundamental kinematic tensors in the contravariant basis. These are also objective.

\subsubsection{Jaumann Strain Rates}

The strain rates conjugate to the Jaumann stress rates of various orders can be derived using the following:

$$
\left[{ }^{(k)} \gamma^{J}\right]=\left[\gamma^{(k)}\right]=\left[\gamma_{(k)}\right] ; k=1,2, \ldots
$$

and hence

$$
\left[{ }^{(k)} \gamma^{J}\right]=\frac{1}{2}\left(\left[\gamma^{(k)}\right]+\left[\gamma_{(k)}\right]\right) \quad ; \quad k=1,2, \ldots
$$

Substituting for $\left[\gamma^{(k)}\right]$ and $\left[\gamma_{(k)}\right]$ from (2.16) and (2.19) in (2.23) and using (2.22) and (2.23) we obtain the following:

$\left[{ }^{(k)} \gamma^{J}\right]=\frac{D}{D t}\left[{ }^{(k-1)} \gamma^{J}\right]-[\bar{W}]\left[{ }^{(k-1)} \gamma^{J}\right]+\left[{ }^{(k-1)} \gamma^{J}\right][\bar{W}]$

with $k=2,3 \ldots$ and

$$
\left[{ }^{(1)} \gamma^{J}\right]=\left[\gamma^{(1)}\right]=\left[\gamma_{(1)}\right]=\frac{1}{2}\left([\bar{L}]+[\bar{L}]^{T}\right)=[\bar{D}]
$$

$\left[{ }^{(j)} \gamma^{J}\right] ; j=1,2, \ldots, n$ are the Jaumann strain rates compatible with the Jaumann stress rates. The Jaumann strain rates are objective. $[\bar{W}]$ is the skew symmetric part of $[\bar{L}]$, i.e. $[\bar{W}]=\frac{1}{2}\left([\bar{L}]-[\bar{L}]^{T}\right)$.

2.5 Convected Time Derivatives of Co-, Contravariant and Jaumann Stress Tensors: Incompressible Matter

In this section we summarize the convected time derivatives of co- and contra-variant Cauchy stress tensors and Jaumann stress tensor for incompressible matter [9] in which $|J|=1$ simplifies the convected time derivative expressions.

\subsubsection{Covariant Basis: Convected Time Derivatives of Covariant Cauchy Stress Tensor}

If $\left[\bar{T}_{(j)}\right] ; j=1,2, \ldots, n$ are the convected time derivatives of orders $j=1,2, \ldots, n$ of the covariant Cauchy stress tensor $\left[\bar{T}_{(0)}\right]$ in the covariant basis and if $\left[T_{[j]}\right]$ $; j=1,2, \ldots, n$ are the material derivatives of orders $j=1,2, \ldots, n$ of the second Piola-Kirchhoff stress tensor $\left[T^{[0]}\right]$, then following reference [9] we can write

$$
\begin{aligned}
& {\left[T_{[k]}\right]=\frac{D}{D t}\left[T_{[k-1]}\right]=[J]^{T}\left[\bar{T}_{(k)}\right][J]} \\
& {\left[\bar{T}_{(k)}\right]=\frac{D}{D t}\left[\bar{T}_{(k-1)}\right]+[\bar{L}]^{T}\left[\bar{T}_{(k-1)}\right]+\left[\bar{T}_{(k-1)}\right][\bar{L}]}
\end{aligned}
$$

in which $k=1,2, \ldots$. It can be easily shown that $\left[\bar{T}_{(j)}\right]$; $j=1,2, \ldots, n$ are objective. 


\subsubsection{Contravariant Basis: Convected Time Deriva- tives of Contravariant Cauchy Stress Tensor}

If $\left[\bar{T}^{(j)}\right] ; j=1,2, \ldots, n$ are the convected time derivatives of orders $j=1,2, \ldots, n$ of the contravariant Cauchy stress tensor $\left[\bar{T}^{(0)}\right]$ in the contravariant basis and if $\left[T^{[j]}\right] ; j=1,2, \ldots, n$ are the material derivatives of orders $j=1,2, \ldots, n$ of the second Piola-Kirchhoff stress tensor $\left[T_{[0]}\right]$, then following reference [9] we can write

$$
\begin{aligned}
& {\left[T^{[k]}\right]=\frac{D}{D t}\left[T^{[k-1]}\right]=[\bar{J}]\left[\bar{T}^{(k)}\right][\bar{J}]^{T}} \\
& {\left[\bar{T}^{(k)}\right]=\frac{D}{D t}\left[\bar{T}^{(k-1)}\right]-[\bar{L}]\left[\bar{T}^{(k-1)}\right]-\left[\bar{T}^{(k-1)}\right][\bar{L}]^{T}}
\end{aligned}
$$

in which $k=1,2, \ldots$. Tensors $\left[\bar{T}^{(j)}\right] ; j=1,2, \ldots, n$ are also objective.

\subsubsection{Jaumann Stress Rates}

In order to define the Jaumann stress rates, i.e. the convected time derivatives of the Jaumann stress tensor, we use the same definitions as used for defining the Jaumann stress tensor, i.e. we assume

$$
\left[{ }^{(k)} \bar{T}^{J}\right]=\left[\bar{T}^{(k)}\right]=\left[\bar{T}_{(k)}\right] ; k=1,2, \ldots \quad \text { (Def.) }
$$

and hence

$$
\left[{ }^{(k)} \bar{T}^{J}\right]=\frac{1}{2}\left(\left[\bar{T}^{(k)}\right]+\left[\bar{T}_{(k)}\right]\right) \quad ; \quad k=1,2, \ldots
$$

Substituting for $\left[\bar{T}^{(k)}\right]$ and $\left[\bar{T}_{(k)}\right]$ from (2.26) and (2.27) in (2.29), using (2.28) and assuming the velocity field to be the same in $\left[\bar{T}^{(k)}\right]$ and $\left[\bar{T}_{(k)}\right]$ definitions (i.e. same $[\bar{L}])$, we obtain

$$
\begin{aligned}
{\left[{ }^{(k)} \bar{T}^{J}\right]=} & \frac{D}{D t}\left(\frac{1}{2}\left(\left[\bar{T}^{(k-1)}\right]+\left[\bar{T}_{(k-1)}\right]\right)\right) \\
& -\frac{1}{2}\left([\bar{L}]-[\bar{L}]^{T}\right)\left[{ }^{(k-1)} \bar{T}^{J}\right] \\
& -\left[{ }^{(k-1)} \bar{T}^{J}\right]\left(\frac{1}{2}\left([\bar{L}]-[\bar{L}]^{T}\right)\right)
\end{aligned}
$$

with $k=1,2, \ldots$. Using (2.29) in (2.30) we can obtain the following recursive relations

$\left[{ }^{(k)} \bar{T}^{J}\right]=\frac{D}{D t}\left[{ }^{(k-1)} \bar{T}^{J}\right]-[\bar{W}]\left[{ }^{(k-1)} \bar{T}^{J}\right]+\left[{ }^{(k-1)} \bar{T}^{J}\right][\bar{W}]$

in which $k=1,2, \ldots$. Tensors $\left[{ }^{(j)} \bar{T}^{J}\right] ; j=1,2, \ldots, n$ are the convected time derivatives of orders $1,2, \ldots$ of the Jaumann stress tensor $\left[{ }^{(0)} \bar{T}^{J}\right]$. The definition (2.31) also requires the deformation to be infinitesimal (not finite) for which case the assumption of the same velocity field, i.e. same $[\bar{L}]$ also holds in the definitions of $\left[\bar{T}^{(k)}\right]$ and $\left[\bar{T}_{(k)}\right]$, essential to derive (2.31). We can show that $\left.{ }^{[(j)} \bar{T}^{J}\right] ; j=1,2, \ldots, n$ are objective.
The first convected time derivative of the Jaumann stress has been used extensively [2], hence we give it a notation similar to upper convected and lower convected rates in the following. If we denote the Jaumann stress tensor by $\left[T^{J}\right]$, i.e.

$$
\begin{aligned}
& {\left[{ }^{(0)} \bar{T}^{J}\right]=\left[T^{J}\right]} \\
& {\left[{ }^{(1)} \bar{T}^{J}\right]=\frac{D}{D t}\left[T^{J}\right]=\frac{D}{D t}\left[T^{J}\right]-[\bar{W}]\left[T^{J}\right]+\left[T^{J}\right][\bar{W}]}
\end{aligned}
$$

then we may drop the superscript ${ }^{J}$ for $\left[T^{J}\right]$ as $\frac{{ }^{J} D}{D t}[T]$ would indeed imply the Jaumann rate of the Jaumann stress tensor. Thus

$$
\frac{{ }^{J} D}{D t}[T]=\frac{D}{D t}[T]-[\bar{W}][T]+[T][\bar{W}]
$$

$\frac{{ }^{J} D}{D t}[T]$ defines the Jaumann stress rate, i.e. first convected time derivative of the Jaumann stress tensor $[T]$.

\subsection{Convected Time Derivatives of Co-, Contra- variant and Jaumann Stress Tensors: Com- pressible Matter}

In this section we summarize the convected time derivatives of co- and contra-variant Cauchy stress tensors and Jaumann stress tensor for compressible matter [9]. In case of compressible matter $|J| \neq 1$, hence the convected time derivative expression contains additional terms compared to the incompressible case.

\subsubsection{Covariant Basis: Convected Time Derivatives of Covariant Cauchy Stress Tensor}

If $\left[\bar{T}_{(j)}\right] ; j=1,2, \ldots, n$ are the convected time derivatives of orders $j=1,2, \ldots, n$ of the covariant Cauchy stress tensor $\left[\bar{T}_{(0)}\right]$ in the covariant basis and if $\left[T_{[j]}\right]$ $; j=1,2, \ldots, n$ are the material derivatives of orders $j=1,2, \ldots, n$ of the second Piola-Kirchhoff stress tensor $\left[T_{[0]}\right]$, then we can write

$$
\begin{aligned}
{\left[T_{[k]}\right]=} & \frac{D}{D t}\left[T_{[k-1]}\right]=|J|[J]^{T}\left[\bar{T}_{(k)}\right][J] \\
{\left[\bar{T}_{(k)}\right]=} & \frac{D}{D t}\left[\bar{T}_{(k-1)}\right]+[\bar{L}]^{T}\left[\bar{T}_{(k-1)}\right]+\left[\bar{T}_{(k-1)}\right][\bar{L}] \\
& +\left[\bar{T}_{(k-1)}\right] \operatorname{tr}([\bar{L}])
\end{aligned}
$$

in which $k=1,2, \ldots$. It can be easily shown that tensors $\left[\bar{T}_{(j)}\right] ; j=1,2, \ldots, n$ are objective.

\subsubsection{Contravariant Basis: Convected Time Deriva- tives of Contravariant Cauchy Stress Tensor}

If $\left[\bar{T}^{(j)}\right] ; j=1,2, \ldots, n$ are the convected time derivatives of orders $j=1,2, \ldots, n$ of the contravariant Cauchy stress tensor $\left[\bar{T}^{(0)}\right]$ in the contravariant basis and if $\left[T^{[j]}\right] ; j=1,2, \ldots, n$ are are the material 
derivatives of orders $j=1,2, \ldots, n$ of the second PiolaKirchhoff stress tensor $\left[T^{[0]}\right]$, then we can write

$$
\begin{aligned}
{\left[T^{[k]}\right]=} & \frac{D}{D t}\left[T^{[k-1]}\right]=|J|[\bar{J}]\left[\bar{T}^{(k)}\right][\bar{J}]^{T} \\
{\left[\bar{T}^{(k)}\right]=} & \frac{D}{D t}\left[\bar{T}^{(k-1)}\right]-[\bar{L}]\left[\bar{T}^{(k-1)}\right]-\left[\bar{T}^{(k-1)}\right][\bar{L}]^{T} \\
& +\left[\bar{T}^{(k-1)}\right] \operatorname{tr}([\bar{L}])
\end{aligned}
$$

in which $k=1,2, \ldots$. Tensors $\left[\bar{T}^{(j)}\right] ; j=1,2, \ldots, n$ are also objective.

\subsubsection{Jaumann Stress Rates}

As in the case of incompressible matter, here also we assume the following

$$
\left[{ }^{(k)} \bar{T}^{J}\right]=\left[\bar{T}^{(k)}\right]=\left[\bar{T}_{(k)}\right] ; k=1,2, \ldots \quad \text { (Def.) }
$$

and hence

$$
\left[{ }^{(k)} \bar{T}^{J}\right]=\frac{1}{2}\left(\left[\bar{T}^{(k)}\right]+\left[\bar{T}_{(k)}\right]\right) \quad ; \quad k=1,2, \ldots
$$

Substituting for $\left[\bar{T}^{(k)}\right]$ and $\left[\bar{T}_{(k)}\right]$ from (2.35) and (2.36) in (2.38), using (2.37) and assuming the velocity field to be the same in $\left[\bar{T}^{(k)}\right]$ and $\left[\bar{T}_{(k)}\right]$ definitions (i.e. same $[\bar{L}]$ ), (following the same procedure as used for incompressible case) we can derive the following:

$$
\begin{aligned}
{\left[{ }^{(k)} \bar{T}^{J}\right]=} & \frac{D}{D t}\left[{ }^{(k-1)} \bar{T}^{J}\right]-[\bar{W}]\left[{ }^{(k-1)} \bar{T}^{J}\right] \\
& +\left[{ }^{(k-1)} \bar{T}^{J}\right][\bar{W}]+\left[{ }^{(k-1)} \bar{T}^{J}\right] \operatorname{tr}([\bar{L}])
\end{aligned}
$$

in which $k=1,2, \ldots$. Using the notations used for incompressible case, the first convected time derivative of the Jaumann stress tensor, i.e. the Jaumann stress rate, for compressible matter can be written as

$$
\frac{{ }^{J} D}{D t}[T]=\frac{D}{D t}[T]-[\bar{W}][T]+[T][\bar{W}]+[T] \operatorname{tr}([\bar{L}])
$$

in which $\frac{{ }^{J} D}{D t}[T]$ is the Jaumann stress rate of the Jaumann stress tensor $[T]$ for compressible matter. $\left[{ }^{(j)} \bar{T}^{J}\right]$ $; j=1,2, \ldots, n$ are the convected time derivatives of orders $1,2, \ldots, n$ of the Jaumann stress tensor $\left[{ }^{(0)} \bar{T}^{J}\right]$ and are objective.

\section{ENTROPY INEQUALITY: DEPENDENT VARIABLES IN THE CONSTITUTIVE THEORY AND THEIR AR- GUMENT TENSORS}

\subsection{Entropy Inequality}

As mentioned earlier, the entropy inequality must provide the basis for deriving the constitutive theories for any deforming matter that is in thermodynamic equilibrium. We could consider entropy inequality in either
Lagrangian or Eulerian description, the conclusions remain unaffected due to the fact that all measures appearing in the entropy inequality are transformable from one description to another through $[J]$ or $[\bar{J}]$. We consider entropy inequality in Lagrangian description $[7,9]$.

$$
\rho_{0}\left(\frac{\partial \Phi}{\partial t}+\eta \frac{\partial \theta}{\partial t}\right)+\frac{q_{i} g_{i}}{\theta}-\sigma_{k i}^{*} \dot{J}_{i k} \leq 0
$$

in which $\rho_{0}$ is material density in the reference configuration, $\Phi$ is Helmholtz free energy density, $\eta$ is entropy density, $\theta$ is temperature, $\boldsymbol{\sigma}^{*}$ is first Piola-Kirchhoff stress tensor, $\boldsymbol{q}$ is the heat vector and $\boldsymbol{g}$ is temperature gradient (all in Lagrangian description, i.e. functions of $x_{i}$ and $t$ ). Since $\boldsymbol{\sigma}^{*}$ can be transformed to any other desired measure, in the following discussion we simply use $\boldsymbol{\sigma}$ for stress.

From the balance of momenta and the first law of thermodynamics we note that the stress tensor and the heat vector are obviously related to the constitution of the matter and hence must be considered as dependent variables in the constitutive theories. In (3.1) we note the appearance of $\Phi$ and $\eta$ as additional dependent variables. Thus, based on conservation laws, the stress tensor, heat vector, Helmholtz free energy density and the entropy density must be considered as dependent variables in the development of the constitutive theory. We note that (3.1) contains material derivative of the Helmholtz free energy density. Thus it is necessary to determine the arguments of $\Phi$ and likewise the arguments of the entropy density $\eta$, stress tensor and heat vector in the entropy inequality. Let $\boldsymbol{\sigma}, \boldsymbol{q}, \Phi$ and $\eta$ and ${ }^{(0)} \overline{\boldsymbol{\sigma}},{ }^{(0)} \overline{\boldsymbol{q}}, \bar{\Phi}$ and $\bar{\eta}$ be the dependent variables in the constitutive theory in Lagrangian and Eulerian descriptions (more specific definitions and choices will be considered in the later sections).

Based on the principles of equipresence [7] we consider all possible measures of deformation as arguments of these dependent variables. The Jacobian of deformation $[J]$ is fundamental in the kinematics of deformation for the matter and hence must be an argument in each of the four dependent variables. Since we are considering fluids and rate theories, $[\dot{J}]$ (time or material derivative of $[J])$ must be an argument as well [9]. Temperature is obviously an argument. In addition to these three, we also consider $\boldsymbol{g}$, the temperature gradient, as an argument. Thus we have

$$
\begin{aligned}
& \boldsymbol{\sigma}=\boldsymbol{\sigma}([J],[\dot{J}], \theta, \boldsymbol{g}) \\
& \boldsymbol{q}=\boldsymbol{q}([J],[\dot{J}], \theta, \boldsymbol{g}) \\
& \Phi=\Phi([J],[\dot{J}], \theta, \boldsymbol{g}) \\
& \eta=\eta([J],[\dot{J}], \theta, \boldsymbol{g})
\end{aligned}
$$

If in (3.2) the independent variables are $\left(x_{i}, t\right)$, then these are in Lagrangian or material description in which 
case $\boldsymbol{\sigma}$ may represent first Piola-Kirchhoff stress, or second Piola-Kirchhoff stress. On the other hand, if the independent variables are $\left(\bar{x}_{i}, t\right)$, then these are in Eulerian or spatial description in which case $\boldsymbol{\sigma}$ may represent contra- or co-variant Cauchy stress tensor or Jaumann stress tensor. Since we have arguments of $\Phi$, we can consider a more detailed form of entropy inequality (3.1) by using

$$
\frac{\partial \Phi}{\partial t}=\frac{\partial \Phi}{\partial J_{i k}} \dot{J}_{i k}+\frac{\partial \Phi}{\partial \dot{J}_{i k}} \ddot{J}_{i k}+\frac{\partial \Phi}{\partial \theta} \dot{\theta}+\frac{\partial \Phi}{\partial g_{i}} \dot{g}_{i}
$$

Substituting (3.3) in (3.1)

$$
\begin{gathered}
\rho_{0}\left(\frac{\partial \Phi}{\partial J_{i k}} \dot{J}_{i k}+\frac{\partial \Phi}{\partial \dot{J}_{i k}} \ddot{J}_{i k}+\frac{\partial \Phi}{\partial \theta} \dot{\theta}+\frac{\partial \Phi}{\partial g_{i}} \dot{g}_{i}+\eta \frac{\partial \theta}{\partial t}\right) \\
+\frac{q_{i} g_{i}}{\theta}-\sigma_{k i}^{*} \dot{J}_{i k} \leq 0
\end{gathered}
$$

or

$$
\begin{aligned}
& \rho_{0} \frac{\partial \Phi}{\partial \dot{J}_{i k}} \ddot{J}_{i k}+\rho_{0}\left(\frac{\partial \Phi}{\partial \theta}+\eta\right) \dot{\theta}+\rho_{0} \frac{\partial \Phi}{\partial g_{i}} \dot{g}_{i} \\
& +\left(\rho_{0} \frac{\partial \Phi}{\partial J_{i k}}-\sigma_{k i}^{*}\right) \dot{J}_{i k}+\frac{q_{i} g_{i}}{\theta} \leq 0
\end{aligned}
$$

In order for (3.5) to hold for arbitrary (but admissible) $[\ddot{J}]$, $\dot{\theta}$ and $\dot{\boldsymbol{g}}$, the following must hold:

$$
\begin{aligned}
& \rho_{0} \frac{\partial \Phi}{\partial \dot{J}_{i k}}=0 \quad \Rightarrow \quad \frac{\partial \Phi}{\partial \dot{J}_{i k}}=0 \\
& \rho_{0} \frac{\partial \Phi}{\partial g_{i}}=0 \quad \Rightarrow \quad \frac{\partial \Phi}{\partial g_{i}}=0 \\
& \rho_{0}\left(\frac{\partial \Phi}{\partial \theta}+\eta\right)=0 \quad \Rightarrow \quad \frac{\partial \Phi}{\partial \theta}+\eta=0 \\
& \left(\rho_{0} \frac{\partial \Phi}{\partial J_{i k}}-\sigma_{k i}^{*}\right) \dot{J}_{i k}+\frac{q_{i} g_{i}}{\theta} \leq 0
\end{aligned}
$$

Equations (3.6) - (3.9) are fundamental relations from the second law of thermodynamics.

\section{Remarks:}

(1) Equations (3.6) imply that $\Phi$ is not a function of $[\dot{J}]$.

(2) Equations (3.7) imply that $\Phi$ is not a function of $\boldsymbol{g}$ either.

(3) Based on (3.8), $\eta$ is not a dependent variable in the constitutive theory as $\eta=-\frac{\partial \Phi}{\partial \theta}$, hence $\eta$ is deterministic from $\Phi$.

(4) The inequality in equation (3.9) is essential in the form it is stated. For example

$$
\rho_{0} \frac{\partial \Phi}{\partial J_{i k}}-\sigma_{k i}^{*}=0 \quad \text { and } \quad \frac{q_{i} g_{i}}{\theta} \leq 0
$$

are inappropriate due to the fact that these imply that $\left[\sigma^{*}\right]$ is not a function of $[\dot{J}]$ (since $\Phi$ is not a function of $[\dot{J}])$ which is contrary to (3.2). We note that (3.9) in its stated form is unable to provide us further details regarding the derivation of the constitutive theory for $\left[\sigma^{*}\right]$ and $\boldsymbol{q}$.

In order to alleviate the situation discussed in remark (4), we consider decomposition of $\left[\sigma^{*}\right]$ into equilibrium stress $\left[{ }_{e} \sigma^{*}\right]$ and deviatoric stress $\left[{ }_{d} \sigma^{*}\right]$

$$
\left[\sigma^{*}\right]=\left[{ }_{e} \sigma^{*}\right]+\left[{ }_{d} \sigma^{*}\right]
$$

in which we have the following:

$$
\begin{aligned}
& {\left[{ }_{e} \sigma^{*}\right]=\left[{ }_{e} \sigma^{*}([J],[0], \theta, 0)\right]} \\
& {\left[{ }_{d} \sigma^{*}\right]=\left[{ }_{d} \sigma^{*}([J],[\dot{J}], \theta, \boldsymbol{g})\right]} \\
& {\left[{ }_{d} \sigma^{*}\right]=\left[{ }_{d} \sigma^{*}([J],[0], \theta, 0)\right]=0}
\end{aligned}
$$

that is, $\left[{ }_{e} \sigma^{*}\right]$ is not a function of $[\dot{J}]$ and $\boldsymbol{g}$, and $\left[{ }_{d} \sigma^{*}\right]$ vanishes when $[\dot{J}]$ and $\boldsymbol{g}$ are zero. Substituting from (3.10) into (3.9) gives

$$
\left(\rho_{0} \frac{\partial \Phi}{\partial J_{i k}}-{ }_{e} \sigma_{k i}^{*}-{ }_{d} \sigma_{k i}^{*}\right) \dot{J}_{i k}+\frac{q_{i} g_{i}}{\theta} \leq 0
$$

or

$$
\left(\rho_{0} \frac{\partial \Phi}{\partial J_{i k}}-{ }_{e} \sigma_{k i}^{*}\right) \dot{J}_{i k}-{ }_{d} \sigma_{k i}^{*} \dot{J}_{i k}+\frac{q_{i} g_{i}}{\theta} \leq 0
$$

Since $\Phi$ is not a function of $[\dot{J}]$ and neither is ${ }_{e} \sigma_{i k}^{*}$ ((3.11)), hence ${ }_{e} \sigma_{i k}^{*}$ must be derivable from

$$
{ }_{e} \sigma_{k i}^{*}=\rho_{0} \frac{\partial \Phi}{\partial J_{i k}} \quad \text { or } \quad\left[{ }_{e} \sigma^{*}\right]^{T}=\rho_{0} \frac{\partial \Phi}{\partial[J]}
$$

Using (3.16), the inequality (3.15) reduces to

$$
{ }_{{ }_{d}} \sigma_{k i}^{*} \dot{J}_{i k}+\frac{q_{i} g_{i}}{\theta} \leq 0
$$

If we assume (as done routinely to derive Fourier heat conduction law [7])

$$
\frac{q_{i} g_{i}}{\theta} \leq 0
$$

then (3.17) is satisfied if the following holds

$$
{ }_{d} \sigma_{k i}^{*} \dot{J}_{i k}>0
$$

Equation (3.19) requires that work expanded due to ${ }_{d} \boldsymbol{\sigma}^{*}$ must be positive. Thus (3.10) can be written as

$$
\begin{aligned}
& \sigma_{i j}^{*}=\rho_{0} \frac{\partial \Phi}{\partial J_{j i}}+{ }_{d} \sigma_{i j}^{*}([J],[\dot{J}], \theta, \boldsymbol{g}) \\
& \Phi=\Phi([J], \theta) \\
& \boldsymbol{q}=\boldsymbol{q}([J],[\dot{J}], \theta, \boldsymbol{g})
\end{aligned}
$$

We note that when $[\dot{J}]$ is an argument of the dependent variables in the constitutive theory: (i) the entropy inequality requires stress decomposition into equilibrium 
stress and deviatoric stress (ii) based on the conditions resulting from the entropy inequality, i.e. (3.20), the equilibrium stress is deterministic from the Helmholtz free energy density, but the constitutive theory for the deviatoric stress is not. Thus, in case of the rate constitutive theory, the entropy inequality does not provide any further mechanism for the development of the constitutive theory beyond (3.20) - (3.22).

Derivation of Fourier heat conduction law for $\boldsymbol{q}$ is straight forward based on (3.18) [7]. A more general derivation based on the theory of generators and invariants is presented in a subsequent section.

\subsection{Further Considerations on Argument Tensors}

We note that in the Eulerian description, transformation of its reference frame by a unimodular (orthogonal) matrix cannot be detected by its subsequent thermomechanical deformation. Thus if $x$-frame changes to $x^{\prime}$ frame via

$$
\begin{aligned}
& \left\{x^{\prime}\right\}=[R]\{x\} \\
\therefore \quad & {\left[J^{\prime}\right]=[J][R]^{T} }
\end{aligned}
$$

Then, based on the principle of frame invariance

$$
\Phi([J], \theta)=\Phi\left(\left[J^{\prime}\right], \theta\right)=\Phi\left([J][R]^{T}, \theta\right)
$$

must hold and likewise, the principle of frame invariance must also hold for the stress tensor and heat vector. But this is only possible if Helmholtz free energy density $\Phi$, the stress tensor and heat vector depend upon the invariants $I_{J}, I_{J}, I I I_{J}$ of $[J]$. Thus, dependence of the variables in the constitutive theory on $[J]$ must be replaced with their dependence on $I_{J}, I_{J}$ and $I I I_{J}$. Furthermore, we note that $[\dot{J}]=[\bar{L}][J],[\bar{D}]=\frac{1}{2}\left([\bar{L}]+[\bar{L}]^{T}\right)$ and $[\bar{W}]=\frac{1}{2}\left([\bar{L}]-[\bar{L}]^{T}\right)$, therefore

$$
[\bar{L}]=[\bar{D}]+[\bar{W}] \quad \text { and } \quad[\dot{J}]=([\bar{D}]+[\bar{W}])[J]
$$

Thus, dependence on $[\dot{J}]$ can be replaced by the dependence on $I_{J}, I_{J}, I I I_{J},[\bar{D}]$ and $[\bar{W}]$. But $[\bar{W}]$ is pure rotation and hence dependence on $[\bar{W}]$ can be eliminated. Thus, we can conclude that Helmholtz free energy density must have dependence on $I_{J}, I_{J}, I I I_{J}$ and $\theta$, and the deviatoric Cauchy stress tensor and heat vector must have dependence on $I_{J}, I_{J}, I I I I_{J},[\bar{D}], \theta$ and $\boldsymbol{g}$. The dependence of Helmholtz free energy density, deviatoric Cauchy stress tensor and heat vector on $I_{J}, I_{J}$, $I_{J}$, though it satisfies the axiom of frame invariance, it is still not so useful due to the fact that $[J]$ and hence $I_{J}$, $I_{J}, I I I_{J}$ are not deterministic in the Eulerian description.

(1) Thus dependence on $I_{J}, I_{J}, I I I_{J}$ must be replaced by some other related measures that are obtainable or defined in the Eulerian description. We note the following from conservation of mass

$$
\rho_{0}=|J| \bar{\rho}=\text { III }_{J} \bar{\rho} \quad \text { or } \quad \text { III }_{J}=\frac{\rho_{0}}{\bar{\rho}}
$$

in which $\rho_{0}$ is density in the reference configuration (hence, constant). Thus dependence on III $_{J}$ can be replaced with dependence on $1 / \bar{\rho}$ or simply $\bar{\rho}$ in the arguments of the Helmholtz free energy density, deviatoric Cauchy stress tensor and heat vector. $I_{J}$ and $I_{J}$ still remain arguments of the Helmholtz free energy density, deviatoric Cauchy stress tensor and heat vector but can not be considered in the development of the constitutive theory for the deviatoric Cauchy stress tensor and heat vector due to the fact that $I_{J}$ and $I_{J}$ are dependent on the components of $[J]$ which are not deterministic (or obtainable) in the Eulerian description as the material particle displacements are not known in the Eulerian description. Thus, we conclude that the deviatoric Cauchy stress tensor and heat vector have density $\bar{\rho}$, symmetric part of the velocity gradient tensor $[\bar{D}]$, temperature $\bar{\theta}$ and temperature gradient $\overline{\boldsymbol{g}}$ as their argument tensors in the Eulerian description.

(2) In the Eulerian description we have three choices: contravariant basis, covariant basis and the Jaumann rates, and hence contravariant Cauchy stress tensor $\left[\bar{\sigma}^{(0)}\right]$, covariant Cauchy stress tensor $\left[\bar{\sigma}_{(0)}\right]$ and the Jaumann stress tensor $\left[{ }^{(0)} \bar{\sigma}^{J}\right]$ (see references $[8,9]$ ) are obvious choices for measures of stress in the constitutive theory.

(3) Recalling the derivations of the convected time derivatives of the Green's strain tensor in the covariant basis, we note that $[\bar{D}]$ is the convected time derivative of order one of the Green's strain in the covariant basis, i.e.

$$
[\bar{D}]=\left[\gamma_{(1)}\right]
$$

$\left[\gamma_{(1)}\right]$ is a fundamental kinematic tensor in covariant basis based on Green's strain tensor, a covariant measure of finite strain.

(4) Likewise if we consider the convected time derivatives of the Almansi strain tensor in contravariant basis, we note that $[\bar{D}]$ is also the convected time derivative of order one of the Almansi strain in contravariant basis, i.e.

$$
[\bar{D}]=\left[\gamma^{(1)}\right]
$$

$\left[\gamma^{(1)}\right]$ is a fundamental kinematic tensor in contravariant basis derived using the Almansi strain tensor, a contravariant measure of finite strain.

(5) We have also shown that

$$
\left[{ }^{(1)} \gamma^{J}\right]=\left[\gamma^{(1)}\right]=\left[\gamma_{(1)}\right]=[\bar{D}]
$$


That is, the first convected time derivative of the Jaumann strain is also a fundamental kinematic tensor. $\left[{ }^{(1)} \gamma^{J}\right]$ is obviously objective.

(6) We have seen that the convected time derivatives of order higher than one of the Green's strain tensor, the Almansi strain tensor as well as higher order Jaumann strain rates can be derived in covariant, contravariant and the Jaumann bases which are fundamental kinematic tensors of various orders in the respective bases. Thus we have

$$
\begin{array}{lll}
{\left[\gamma^{(j)}\right]} & ; & j=1,2, \ldots, n \\
{\left[\gamma_{(j)}\right]} & ; & j=1,2, \ldots, n \\
{\left[{ }^{(j)} \gamma^{J}\right]} & ; & j=1,2, \ldots, n
\end{array}
$$

Hence, instead of considering $[\bar{D}]$ or $\left[\gamma^{(1)}\right]$ or $\left[\gamma_{(1)}\right]$ as argument tensor in the constitutive theory, we can generalize the choice by replacing $[\bar{D}]$ with $\left[\gamma^{(j)}\right] ; j=1,2, \ldots, n$ or $\left[\gamma_{(j)}\right] ; j=1,2, \ldots, n$ or $\left.{ }^{[(j)} \gamma^{J}\right] ; j=1,2, \ldots, n$ depending upon whether the basis is contra- or co-variant or we are considering the Jaumann rates.

(7) From the Maxwell model, Giesekus model, Oldroyd-B model etc. we note that these models contain convected time derivatives of orders one and zero of the stress tensor. Thus these must be derivable by considering the first convected time derivative of the deviatoric Cauchy stress tensor as a dependent variable in the constitutive theory in which the convected time derivative of order zero of the deviatoric Cauchy stress tensor is an argument tensor (see later sections). In the work presented here we generalize this concept and consider the convected time derivative of order ' $m$ ' of the chosen deviatoric Cauchy stress tensor (co- or contra-variant basis or Jaumann) as a dependent variable in the constitutive theory with convected time derivatives of up to order ' $m-1$ ' of the same deviatoric stress tensor as its arguments in addition to the other argument tensors.

(8) Thus, we need to consider constitutive theories for $\left(\left[{ }_{d} \bar{\sigma}^{(m)}\right], \overline{\boldsymbol{q}}^{(0)}, \bar{\Phi}\right),\left(\left[{ }_{d} \bar{\sigma}_{(m)}\right], \overline{\boldsymbol{q}}_{(0)}, \bar{\Phi}\right)$ and $\left(\left[{ }^{(m)_{d}} \bar{\sigma}^{J}\right]\right.$, $\left.{ }^{(0)} \overline{\boldsymbol{q}}^{J}, \bar{\Phi}\right)$ in contravariant, covariant and Jaumann bases.

(9) Remarks (7) and (8) help us in finalizing the dependent variables and their argument tensors in the three bases. In order to make the following presentation and subsequent derivations of rate constitutive theories for the deviatoric stress and heat vector compact, we introduce the following notations:

Let $\left[{ }^{(k)} \bar{\sigma}\right] ; k=0,1, \ldots, m,{ }^{(0)} \overline{\boldsymbol{q}}$ and $\left[{ }^{(j)} \gamma\right] ; j=$ $1,2, \ldots, n$ be the Cauchy stress tensor rates, heat vector and the corresponding convected time derivatives of the strain tensor in the chosen basis, and let $\left[{ }^{(0)} \bar{\sigma}\right]=\left[{ }^{(0)} \bar{\sigma}\right]+\left[{ }^{(0)} \bar{d}\right]$ be the decomposition of the Cauchy stress tensor into equilibrium stress and the deviatoric stress.

By choosing $\left[{ }^{(k)} \bar{\sigma}\right] ; k=0,1, \ldots, m,{ }^{(0)} \overline{\boldsymbol{q}}$ and $\left[{ }^{(j)} \gamma\right]$; $j=1,2, \ldots, n$ as $\left(\left[\bar{\sigma}^{(k)}\right] ; k=0,1, \ldots, m, \overline{\boldsymbol{q}}^{(0)}\right.$ and $\left[\gamma^{(j)}\right]$ $; j=1,2, \ldots, n)$ or $\left(\left[\bar{\sigma}_{(k)}\right] ; k=0,1, \ldots, m, \overline{\boldsymbol{q}}_{(0)}\right.$ and $\left.\left[\gamma_{(j)}\right] ; j=1,2, \ldots, n\right)$ or $\left(\left[{ }^{(k)} \bar{\sigma}^{J}\right] ; k=0,1, \ldots, m,{ }^{(0)} \overline{\boldsymbol{q}}^{J}\right.$ and $\left.\left[{ }^{(j)} \gamma^{J}\right] ; j=1,2, \ldots, n\right)$ we can easily obtain various details of rate theories in contravariant basis, covariant basis and using Jaumann rates.

\section{CONSTITUTIVE THEORY FOR THE EQUILIBRIUM STRESS TENSOR}

In order to present the derivation of the constitutive theory for the equilibrium stress tensor $\left[{ }_{e}^{(0)} \bar{\sigma}\right]$, we must consider a specific choice of basis (due to the fact that $\left[{ }_{e} \sigma^{*}\right]$ in (3.16) must be transfered to the basis of choice).

\subsection{Compressible Matter}

Consider contravariant basis, i.e. we want to derive a constitutive theory for $\left[e_{e} \bar{\sigma}^{(0)}\right]$. We begin with (3.16) resulting from the entropy inequality

$$
\left[{ }_{e} \sigma^{*}\right]^{T}=\rho_{0} \frac{\partial \Phi(\rho, \theta)}{\partial[J]}
$$

and use

$$
\left[e^{(0)}\right]=|J|^{-1}\left[{ }_{e} \sigma^{*}\right]^{T}[J]^{T}
$$

Following the derivations presented in reference [7] we obtain

$$
\left[e^{\bar{\sigma}^{(0)}}\right]=\bar{p}(\bar{\rho}, \bar{\theta})[I]
$$

From (4.3) we note that $\left[e^{(0)}\right]$ is independent of the basis, i.e.

$$
\left[{ }_{e} \bar{\sigma}^{(0)}\right]=\left[e_{e} \bar{\sigma}_{(0)}\right]=\left[{ }^{(0)} \bar{\sigma}^{J}\right]=\bar{p}(\bar{\rho}, \bar{\theta})[I]
$$

in which $\bar{p}(\bar{\rho}, \bar{\theta})$ is thermodynamic pressure defined by the equation of state and is deterministic from the deformation field. If we assume compressive pressure to be positive, then $\bar{p}(\bar{\rho}, \bar{\theta})$ in (4.4) can be replaced by $-\bar{p}(\bar{\rho}, \bar{\theta})$.

\subsection{Incompressible Matter}

For incompressible matter $\bar{\rho}=\rho_{0}=$ constant which implies that $|J|=1$ and hence $\Phi=\Phi(\theta)$ and therefore $\frac{\partial \Phi}{\partial[J]}=0$ in (4.1). Thus, for incompressible matter, $\left[{ }_{e} \sigma^{*}\right]$ can not be determined using the derivation used for compressible case. The incompressibility condition

$$
\operatorname{tr}([\bar{D}])=\operatorname{tr}([\bar{L}])=\operatorname{tr}\left([\dot{J}][J]^{-1}\right)=\dot{J}_{i k}\left(J^{-1}\right)_{k i}=0
$$

must be enforced. Based on (4.5) we can add

$$
p \dot{J}_{i k}\left(J^{-1}\right)_{k i}=p(\theta) \dot{J}_{i k}\left(J^{-1}\right)_{k i}=0
$$


to the entropy inequality to derive equilibrium stress $\left[e \bar{\sigma}^{(0)}\right]$ for incompressible matter. In (4.6), $p(\theta)$ is an arbitrary Lagrange multiplier. Following [7] we can obtain (in contravariant basis)

$$
\left[e^{(0)}\right]=\bar{p}(\bar{\theta})[I]
$$

$\bar{p}(\bar{\theta})$ is mechanical pressure. $\bar{p}(\bar{\theta})$ is not deterministic from the deformation field. If we define the compressive pressure to be positive, then $\bar{p}(\bar{\theta})$ in (4.7) can be replaced by $-\bar{p}(\bar{\theta})$. In this case also, we note that the equilibrium stress is independent of the basis, i.e.

$$
\left[e^{\bar{\sigma}^{(0)}}\right]=\left[e^{\bar{\sigma}_{(0)}}\right]=\left[{ }^{(0)} \bar{\sigma}^{J}\right]=\bar{p}(\bar{\theta})[I]
$$

\section{CONSIDERATIONS IN THE CONSTITUTIVE THE- ORIES FOR DEVIATORIC CAUCHY STRESS TEN- SOR AND HEAT VECTOR: COMPRESSIBLE MAT- TER}

We make some remarks in the following that are helpful in understanding the approach used in the present work. This is followed by definitions of dependent variables and their argument tensors.

\subsection{Remarks}

(1) $\left[{ }^{(j)} \gamma\right] ; j=1,2, \ldots, n$ are fundamental symmetric kinematic tensors of rank two and $\overline{\boldsymbol{g}}$ is a tensor of rank one. $\left[{ }^{(k)}{ }_{d} \bar{\sigma}\right] ; k=0,1, \ldots, m$ are symmetric tensors of rank two and $\bar{\rho}, \bar{\theta}$ are tensors of rank zero.

(2) $\left[{ }^{(j)} \gamma\right] ; j=1,2, \ldots, n,\left[{ }^{(k)}{ }_{d} \bar{\sigma}\right] ; k=0,1, \ldots, m$ and $\overline{\boldsymbol{g}}$ have their own generators and invariants but also there exist combined generators and invariants between them.

(3) In the case of homogeneous isotropic compressible matter, the equilibrium stress is completely deterministic from the entropy inequality once we define Helmholtz free energy density in terms of the invariants of the chosen strain measure. This yields thermodynamic pressure $\bar{p}(\bar{\rho}, \bar{\theta})$. In the case of incompressible matter, the equilibrium stress is also derived from the entropy inequality in conjunction with incompressibility constraint, however, the equilibrium stress is not a function of the Helmholtz free energy density and thus it is not deterministic from the deformation field [7]. Furthermore, the second law of thermodynamics only restricts the work expanded due to the deviatoric stress to be positive but provides no mechanism for determining the constitutive theory for the deviatoric stress.

(4) The theory of generators and invariants $[14,15]$ provides a continuum mechanics foundation to derive constitutive equations for the deviatoric
Cauchy stress tensor and heat vector. In this approach we determine the combined generators of the argument tensors of the dependent variable in the constitutive theory that form integrity or minimal basis. The dependent variables in the constitutive theory are expressed as a linear combination of the combined generators of the argument tensors. In the constitutive theory presented here we consider $\left[{ }^{(m)}{ }_{d} \bar{\sigma}\right],{ }^{(0)} \overline{\boldsymbol{q}}$ and $\bar{\Phi}$ as dependent variables in the constitutive theory.

(a) $\bar{\rho}(\overline{\boldsymbol{x}}, t), \bar{\theta}(\overline{\boldsymbol{x}}, t),\left[{ }^{(k)}{ }_{d} \bar{\sigma}(\overline{\boldsymbol{x}}, t)\right] ; k=0,1, \ldots, m-1$, $\left.{ }^{[(j)} \gamma(\overline{\boldsymbol{x}}, t)\right] ; j=1,2, \ldots, n$ and $\overline{\boldsymbol{g}}(\overline{\boldsymbol{x}}, t)$ are considered as argument tensors of $\left[{ }^{(m)} \bar{d}\right]$.

(b) We also consider $\bar{\rho}(\overline{\boldsymbol{x}}, t), \bar{\theta}(\overline{\boldsymbol{x}}, t),\left[{ }^{(k)} \bar{d} \bar{\sigma}(\overline{\boldsymbol{x}}, t)\right]$; $k=0,1, \ldots, m-1,\left[{ }^{(j)} \gamma(\overline{\boldsymbol{x}}, t)\right] ; j=1,2, \ldots, n$ and $\overline{\boldsymbol{g}}(\overline{\boldsymbol{x}}, t)$ as argument tensors of the heat vector $^{(0)} \overline{\boldsymbol{q}}$.

Thus, in case of $\left[{ }^{(m)}{ }_{d} \bar{\sigma}\right]$, a symmetric tensor of rank two, we need combined generators of the tensors $\left[{ }^{(k)} \bar{d} \bar{\sigma}\right] ; k=0,1, \ldots, m-1,\left[{ }^{(j)} \gamma\right] ; j=1,2, \ldots, n$ and $\overline{\boldsymbol{g}}$ that are symmetric tensors of rank two in addition to the combined invariants of the same argument tensors. Whereas ${ }^{(0)} \overline{\boldsymbol{q}}$ is a tensor of rank one, hence in this case we need combined generators of the tensors $\left[{ }^{(k)}{ }_{d} \bar{\sigma}\right] ; k=0,1, \ldots, m-1,\left[{ }^{(j)} \gamma\right]$ $; j=1,2, \ldots, n$ and $\overline{\boldsymbol{g}}$ that are tensors of rank one. We express $\left[{ }^{(m)} \bar{\sigma}\right]$ and ${ }^{(0)} \overline{\boldsymbol{q}}$ as a linear combination of the combined generators of their argument tensors. The coefficients used in the linear combination are functions of $\bar{\rho}, \bar{\theta}$ and the combined invariants of the argument tensors and are determined using Taylor series expansion of each coefficient about a known configuration.

(5) Based on (4), the key element in the theory of generators and invariants is the determination of the minimal basis, i.e. integrity, using the combined generators of the argument tensors and of course, determination of the combined invariants. For example, if we consider $[T([S])]$, where $[T]$ and $[S]$ are symmetric tensors of rank two, which obey the invariance

$$
\left[T\left([R][S][R]^{T}\right)\right]=[R][T([S])][R]^{T}
$$

then the tensor $[T]$ has the following form:

$$
[T]=\alpha_{0}[I]+\alpha_{1}[S]+\alpha_{2}[S]^{2}
$$

where $\alpha_{0}, \alpha_{1}$ and $\alpha_{2}$ are functions of the invariants of $[S]$, i.e.

$$
\begin{aligned}
& i_{S}=\operatorname{tr}([S]) \\
& i i_{S}=\operatorname{tr}\left([S]^{2}\right) \\
& i i i_{S}=\operatorname{tr}\left([S]^{3}\right)
\end{aligned}
$$


called principal invariants, or the invariants $I_{s}, I_{s}$ and $\mathrm{III}_{s}$ from the characteristic equation of $[S]$. The tensors $[I],[S],[S]^{2}$ are generators of the tensor $[T]$ and form the minimal basis.

(6) If the arguments of $[T]$ consist of more than one tensor (could be of different rank), then a linear combination like (5.2) would contain all combined generators (of the same rank as $[T]$ ) of the argument tensors of $[T]$ and likewise the coefficients in the linear combination would be functions of the argument tensors of rank zero and the combined invariants of the argument tensors of rank one and two. For details on the combined generators and invariants for various combinations of the argument tensors of various ranks see references $[14,15]$.

(7) Based on the remarks presented above, we now have a basis for deriving constitutive theory for the deviatoric stress tensor as well as the heat vector. Details of the constitutive theory are presented using $\left[{ }^{(k)} \bar{\sigma} \bar{\sigma}\right] ; k=0,1, \ldots, m,{ }^{(0)} \overline{\boldsymbol{q}}$ and $\left[{ }^{(j)} \gamma\right] ;$ $j=1,2, \ldots, n$ and $\bar{\rho}, \bar{\theta}$ as argument tensors of the dependent variables. These can be made basis specific by appropriate choices of the stress rates and strain rates.

\subsection{Dependent Variables and their Argument Ten- sors}

Let $\left[{ }^{(k)}{ }_{d} \bar{\sigma}\right] ; k=0,1, \ldots, m,{ }^{(0)} \overline{\boldsymbol{q}}$ and $\left[{ }^{(j)} \gamma\right] ;$ $j=1,2, \ldots, n$ be the convected time derivatives of the deviatoric Cauchy stress tensor, heat vector and the corresponding convected time derivatives of the strain tensor in the chosen basis. Let $\bar{\rho}, \bar{\theta}$ and $\overline{\boldsymbol{g}}$ be the density, temperature and temperature gradient, all in the current configuration.

We consider $\left[{ }^{(m)} \bar{d} \bar{\sigma}\right],{ }^{(0)} \overline{\boldsymbol{q}}$ and $\bar{\Phi}$ as dependent variables in the constitutive theory. $\left[{ }^{(k)} \bar{\sigma} \bar{\sigma}\right] ; k=0,1, \ldots, m-$ $1,\left[{ }^{(j)} \gamma\right] ; j=1,2, \ldots, n, \bar{\rho}, \bar{\theta}$ and $\overline{\boldsymbol{g}}$ are argument tensors of $\left[{ }^{(m)} \bar{\sigma} \bar{\sigma}\right]$ and ${ }^{(0)} \overline{\boldsymbol{q}}$. The argument tensors of $\bar{\Phi}$ for compressible case are $\bar{\rho}$ and $\bar{\theta}$ and for incompressible case, only $\bar{\theta}$ is the argument tensor of $\bar{\Phi}$. Thus, we have the following for the compressible and incompressible thermoviscoelastic fluids considered here.

\section{Compressible Thermoviscoelastic Fluids:}

$$
\begin{aligned}
& \bar{\Phi}=\bar{\Phi}(\bar{\rho}(\overline{\boldsymbol{x}}, t), \bar{\theta}(\overline{\boldsymbol{x}}, t)) \\
& {\left[{ }^{(0)} \bar{\sigma}\right]=\left[{ }_{e}^{(0)} \bar{\sigma}(\bar{\rho}(\overline{\boldsymbol{x}}, t), \bar{\theta}(\overline{\boldsymbol{x}}, t))\right]+\left[{ }^{\left({ }^{0}\right)}{ }_{d} \bar{\sigma}\right]} \\
& {\left[{ }^{(m)} \bar{\sigma}\right]=\left[{ } _ { d } ^ { ( m ) } \overline { \sigma } \left(\bar{\rho}(\overline{\boldsymbol{x}}, t),\left[{ }^{(k)} \bar{\sigma}(\overline{\boldsymbol{x}}, t)\right] ; k=0,1, \ldots, m-1,\right.\right.} \\
& \\
& \left.\left.\quad\left[{ }^{(j)} \gamma(\overline{\boldsymbol{x}}, t)\right] ; j=1,2, \ldots, n, \bar{\theta}(\overline{\boldsymbol{x}}, t), \overline{\boldsymbol{g}}(\overline{\boldsymbol{x}}, t)\right)\right]
\end{aligned}
$$

$$
\begin{array}{r}
{ }^{(0)} \overline{\boldsymbol{q}}={ }^{(0)} \overline{\boldsymbol{q}}\left(\bar{\rho}(\overline{\boldsymbol{x}}, t),\left[{ }^{(k)}{ }_{d} \bar{\sigma}(\overline{\boldsymbol{x}}, t)\right] ; k=0,1 \ldots, m-1,\right. \\
\left.\quad\left[{ }^{(j)} \gamma(\overline{\boldsymbol{x}}, t)\right] ; j=1,2 \ldots, n, \bar{\theta}(\overline{\boldsymbol{x}}, t), \overline{\boldsymbol{g}}(\overline{\boldsymbol{x}}, t)\right)
\end{array}
$$

$\left[e^{*} \sigma^{T}=\rho(\boldsymbol{x}) \frac{\partial \Phi(\rho(\boldsymbol{x}, t), \theta(\boldsymbol{x}, t))}{\partial[J(\boldsymbol{x}, t)]}\right.$

where $\rho(\boldsymbol{x})=\rho$. Using (5.4) - (5.8), we present developments of the rate constitutive theories of various orders. By choosing $\left[{ }^{(k)} \bar{\sigma}\right] ; k=0,1, \ldots, m,{ }^{(0)} \overline{\boldsymbol{q}}$ and $\left[{ }^{(j)} \gamma\right] ; j=1,2, \ldots, n$ as $\left(\left[\bar{\sigma}^{(k)}\right] ; k=0,1, \ldots, m, \overline{\boldsymbol{q}}^{(0)}\right.$ and $\left.\left[\gamma^{(j)}\right] ; j=1,2, \ldots, n\right)$ or $\left(\left[\bar{\sigma}_{(k)}\right] ; k=0,1, \ldots, m, \overline{\boldsymbol{q}}_{(0)}\right.$ and $\left.\left[\gamma_{(j)}\right] ; j=1,2, \ldots, n\right)$ or $\left(\left[{ }^{(k)} \bar{\sigma}^{J}\right] ; k=0,1, \ldots, m\right.$, ${ }^{(0)} \overline{\boldsymbol{q}}^{J}$ and $\left.\left[{ }^{(j)} \gamma^{J}\right] ; j=1,2, \ldots, n\right)$ in (5.4) - (5.8) and following the derivations presented in the subsequent sections, we can obtain rate theories in contravariant basis, covariant basis and using Jaumann rates.

\section{Incompressible Thermoviscoelastic Fluids:}

If the matter is incompressible, then $\bar{\rho}=\rho_{0}=$ constant, hence $\bar{\rho}$ drops out of all quantities in (5.4) (5.8). However $\bar{\rho}=\rho_{0}=$ constant implies that $|J|=1$, hence for this case we have $\bar{\Phi}=\bar{\Phi}(\bar{\theta}(\overline{\boldsymbol{x}}, t))$ and therefore $\frac{\partial \Phi}{\partial[J]}=0$. Thus $\left[{ }_{e} \sigma^{*}\right]$ can not be derived using (5.8) if the matter is incompressible. For incompressible thermoviscoelastic fluids (5.4) - (5.8) reduces to

$$
\begin{aligned}
& \bar{\Phi}=\bar{\Phi}(\bar{\theta}(\overline{\boldsymbol{x}}, t)) \\
& {\left[{ }^{(0)} \bar{\sigma}\right]=\left[{ }_{e}^{(0)} \bar{\sigma}(\bar{\theta}(\overline{\boldsymbol{x}}, t))\right]+\left[{ }_{d}^{(0)} \bar{\sigma}\right]} \\
& {\left[{ }^{(m)} \bar{d}\right]=\left[{ } ^ { ( m ) } { } _ { d } \overline { \sigma } \left(\left[{ }^{(k)}{ }_{d} \bar{\sigma}(\overline{\boldsymbol{x}}, t)\right] ; k=0,1, \ldots, m-1,\right.\right.} \\
& \left.\left.\left.{ }^{(j)} \gamma(\overline{\boldsymbol{x}}, t)\right] ; j=1,2, \ldots, n, \bar{\theta}(\overline{\boldsymbol{x}}, t), \overline{\boldsymbol{g}}(\overline{\boldsymbol{x}}, t)\right)\right] \\
& { }^{(0)} \overline{\boldsymbol{q}}={ }^{(0)} \overline{\boldsymbol{q}}\left(\left[{ }^{(k)}{ }_{d} \bar{\sigma}(\overline{\boldsymbol{x}}, t)\right] ; k=0,1, \ldots, m-1,\right. \\
& \left.\left[{ }^{(j)} \gamma(\overline{\boldsymbol{x}}, t)\right] ; j=1,2 \ldots, n, \bar{\theta}(\overline{\boldsymbol{x}}, t), \overline{\boldsymbol{g}}(\overline{\boldsymbol{x}}, t)\right)
\end{aligned}
$$

$$
\frac{\partial \Phi(\theta(\boldsymbol{x}, t))}{\partial[J(\boldsymbol{x}, t)]}=0
$$

The constitutive theories for $\left[{ }^{(m)} \bar{\sigma}\right]$ and ${ }^{(0)} \overline{\boldsymbol{q}}$ are derived using (5.6) and (5.7) or (5.11) and (5.12), and can be converted to contravariant basis, covariant basis or Jaumann rate by replacing $\left[{ }^{(m)}{ }_{d} \bar{\sigma}\right],\left[{ }^{(0)} \bar{\sigma}\right],{ }^{(0)} \overline{\boldsymbol{q}}$ and $\left[{ }^{(j)} \gamma\right]$ $; j=1,2, \ldots, n$ with the appropriate measures in the chosen basis. In the following sections we consider details of the development of rate constitutive theories for 
deviatoric Cauchy stress and heat vector for both compressible as well as incompressible thermoviscoelastic fluids.

\section{RATE CONSTITUTIVE THEORY OF ORDERS ' $M$ ' AND ' $N$ ' FOR THE DEVIATORIC CAUCHY STRESS TENSOR AND THE HEAT VECTOR: COMPRESS- IBLE THERMOVISCOELASTIC FLUIDS}

Consider a deforming volume of compressible thermoviscoelastic fluid in the current configuration. We derive the rate constitutive theory of orders ' $m$ ' and ' $n$ ' for the deviatoric Cauchy stress tensor $\left[{ }^{(0)} \bar{d}\right]$ and heat vector ${ }^{(0)} \overline{\boldsymbol{q}}$ using ((5.6) and (5.7))

$$
\begin{array}{r}
{\left[{ }_{d}^{(m)} \bar{\sigma}\right]={ }^{(m)} \bar{d} \bar{\sigma}\left(\bar{\rho},\left[{ }^{(k)} \bar{\sigma}\right] ; k=0,1, \ldots, m-1,\right.} \\
\left.\left.\left[{ }^{(j)} \gamma\right] ; j=1,2, \ldots, n, \bar{\theta}, \overline{\boldsymbol{g}}\right)\right] \\
{ }^{(0)} \overline{\boldsymbol{q}}={ }^{(0)} \overline{\boldsymbol{q}}\left(\bar{\rho},\left[{ }^{(k)}{ }_{d} \bar{\sigma}\right] ; k=0,1, \ldots, m-1,\right. \\
\left.\left[{ }^{(j)} \gamma\right] ; j=1,2, \ldots, n, \bar{\theta}, \overline{\boldsymbol{g}}\right)
\end{array}
$$

\subsection{Constitutive Theory of Orders $(\boldsymbol{m}, \boldsymbol{n})$ for $\left[{ }^{(m)} \bar{d} \bar{\sigma}\right]$}

Let $\left[{ }^{\sigma} G^{i}\right] ; i=1,2, \ldots, N$ be the combined generators (of $\left[{ }^{(m)} \bar{\sigma}\right]$ ) of the argument tensors $\left[{ }^{(k)} \bar{\sigma}\right] ; k=$ $0,1, \ldots, m-1,\left[{ }^{(j)} \gamma\right] ; j=1,2, \ldots, n$ and $\bar{g}$ that are symmetric tensors of rank two, and let ${ }^{q \sigma}{ }_{\sim}{ }^{j} ; j=1,2, \ldots, M$ be the combined invariants of the same argument tensors. Then, we can express $\left[{ }^{(m)} \bar{\sigma}\right]$ as a linear combination of the generators $\left[{ }^{\sigma} G^{i}\right] ; i=1,2, \ldots, N$ and the identity tensor $[I]$ in the current configuration.

$$
\left[{ }_{d}^{(m)} \bar{\sigma}\right]={ }^{\sigma} \alpha^{0}[I]+\sum_{i=1}^{N}{ }^{\sigma} \alpha^{i}\left[{ }^{\sigma} G^{i}\right]
$$

The coefficients ${ }^{\sigma} \alpha^{i} ; i=0,1, \ldots, N$ in (6.3) are functions of density $\bar{\rho}$, temperature $\bar{\theta}$ and the combined invariants ${ }^{q \sigma}{ }_{\sim}^{I^{j}} ; j=1,2, \ldots, M$ in the current configuration. To determine the material coefficients from ${ }^{\sigma} \alpha^{i}$; $i=0,1, \ldots, N$ in (6.3) which are defined in the current configuration, we consider the Taylor series expansion of each ${ }^{\sigma} \alpha^{i} ; i=0,1, \ldots, N$ about a known configuration $\underline{\Omega}$ in $\bar{\theta}$ and ${ }^{q \sigma} \underline{\sim}^{j} ; j=1,2, \ldots, M$ and retain only up to linear terms in $\bar{\theta}$ and the invariants.

$$
\begin{aligned}
\sigma^{\sigma} \alpha^{i}= & \left.{ }^{\sigma} \alpha^{i}\right|_{\underline{\Omega}}+\left.\sum_{j=1}^{M} \frac{\partial\left({ }^{\sigma} \alpha^{i}\right)}{\partial\left({ }^{q \sigma} \underline{\sim}^{j}\right)}\right|_{\underline{\Omega}}\left({ }^{q \sigma} \stackrel{\sim}{I}^{j}-\left({ }^{q \sigma}{\underset{\sim}{I}}^{j}\right)_{\underline{\Omega}}\right) \\
& +\left.\frac{\partial\left({ }^{\sigma} \alpha^{i}\right)}{\partial \bar{\theta}}\right|_{\underline{\Omega}}\left(\bar{\theta}-\bar{\theta}_{\underline{\Omega}}\right) ; i=0,1, \ldots, N
\end{aligned}
$$

$$
\left.{ }^{\sigma} \alpha^{i}\right|_{\underline{\Omega}},\left.\quad \frac{\partial\left(\sigma^{\sigma} \alpha^{i}\right)}{\partial\left(q \sigma I^{j}\right)}\right|_{\underline{\Omega}} ; j=1,2, \ldots, M \text { and }\left.\frac{\partial\left({ }^{\sigma} \alpha^{i}\right)}{\partial \bar{\theta}}\right|_{\underline{\Omega}}
$$
$; i=0,1, \ldots, N$ are functions of $\bar{\rho}_{\Omega}, \bar{\theta}_{\Omega}$ and $\left({ }^{q \sigma}{ }^{I^{j}}\right)_{\underline{\Omega}} ; j=1,2, \ldots, M$ whereas in (6.4), ${ }^{\sigma} \alpha^{i}=$ ${ }^{\sigma} \alpha^{i}\left(\bar{\rho}_{\underline{\Omega}}, \bar{\theta}_{\underline{\Omega}},\left({ }^{q \sigma} I_{\sim}{ }^{j}\right)_{\underline{\underline{\Omega}}} ; j=1,2, \ldots, M, \bar{\theta},{ }^{q \sigma} \underline{\sim}^{j} ; j=\right.$ $1,2, \ldots, M) ; i=0,1, \ldots, N$. When (6.4) is substituted in (6.3), we obtain the final form of the most general rate constitutive theory of orders $(m, n)$ for $\left[{ }^{(m)} \bar{d} \bar{\sigma}\right]$ for compressible thermoviscoelastic fluids. Details are given the following. Substituting from (6.4) into (6.3)

$$
\begin{aligned}
{\left[{ }^{\left.(m)_{d} \bar{\sigma}\right]}\right.} & =\left(\left.{ }^{\sigma} \alpha^{0}\right|_{\underline{\Omega}}+\left.\sum_{j=1}^{M} \frac{\partial\left({ }^{\sigma} \alpha^{0}\right)}{\partial\left({ }^{q \sigma} \underline{\sim}^{j}\right)}\right|_{\underline{\Omega}}\left({ }^{q \sigma} \underline{\sim}^{j}-\left({ }^{q \sigma} \underline{\sim}^{j}\right)_{\underline{\Omega}}\right)\right. \\
& \left.+\left.\frac{\partial\left({ }^{\sigma} \alpha^{0}\right)}{\partial \bar{\theta}}\right|_{\underline{\Omega}}\left(\bar{\theta}-\bar{\theta}_{\underline{\Omega}}\right)\right)[I]+ \\
& \sum_{i=1}^{N}\left(\left.{ }^{\sigma} \alpha^{i}\right|_{\underline{\Omega}}+\left.\sum_{j=1}^{M} \frac{\partial\left({ }^{\sigma} \alpha^{i}\right)}{\partial\left({ }^{q \sigma} \underline{I}^{j}\right)}\right|_{\underline{\Omega}}\left({ }^{q \sigma} \underline{\sim}^{j}-\left({ }^{q \sigma} \underline{\sim}^{j}\right)_{\underline{\Omega}}\right)\right. \\
& \left.+\left.\frac{\partial\left({ }^{\sigma} \alpha^{i}\right)}{\partial \bar{\theta}}\right|_{\underline{\Omega}}\left(\bar{\theta}-\bar{\theta}_{\underline{\Omega}}\right)\right)\left[{ }^{\sigma} G^{i}\right]
\end{aligned}
$$

Collecting coefficients (only those defined in the known configuration $\underline{\Omega}$ ) of $[I],{ }^{q \sigma}{\underset{\sim}{I}}^{j}[I],\left[{ }^{\sigma} G^{i}\right],{ }^{q \sigma}{\underset{\sim}{I}}^{j}\left[{ }^{\sigma} G^{i}\right]$, $\left(\bar{\theta}-\bar{\theta}_{\underline{\Omega}}\right)\left[{ }^{\sigma} G^{i}\right]$ and $\left(\bar{\theta}-\bar{\theta}_{\underline{\Omega}}\right)[I]$ in (6.5) and defining

$$
\left.\bar{\sigma}^{0}\right|_{\underline{\Omega}}=\left.{ }^{\sigma} \alpha^{0}\right|_{\underline{\Omega}}-\left.\sum_{j=1}^{M} \frac{\partial\left({ }^{\sigma} \alpha^{0}\right)}{\partial\left({ }^{q \sigma} \underline{I}^{j}\right)}\right|_{\underline{\Omega}}\left({ }^{q \sigma} \underline{I}^{j}\right)_{\underline{\Omega}}
$$

and the material coefficients

$$
\begin{aligned}
& \underline{a}_{j}=\left.\frac{\partial\left({ }^{\sigma} \alpha^{0}\right)}{\partial\left({ }^{\sigma \sigma} \underline{\sim}^{j}\right)}\right|_{\underline{\Omega}} ; j=1,2, \ldots, M \\
& { }^{\sigma} \underline{b}_{i}=\left.{ }^{\sigma} \alpha^{i}\right|_{\underline{\Omega}}-\left.\sum_{j=1}^{M} \frac{\partial\left({ }^{\sigma} \alpha^{i}\right)}{\partial\left({ }^{\sigma \sigma} \underline{\sim}^{j}\right)}\right|_{\underline{\Omega}} ; i=1,2, \ldots, N \\
& { }^{\sigma} \mathcal{C}_{i j}=\left.\frac{\partial\left({ }^{\sigma} \alpha^{i}\right)}{\partial\left({ }^{q \sigma} \underline{\sim}^{j}\right)}\right|_{\underline{\Omega}} ; i=1,2, \ldots, N ; j=1,2, \ldots, M \\
& { }_{\underline{d_{i}}}=\left.\frac{\partial\left({ }^{\sigma} \alpha^{i}\right)}{\partial \bar{\theta}}\right|_{\underline{\Omega}} ; i=1,2, \ldots, N \\
& \left(\alpha_{t m}\right)_{\underline{\Omega}}=-\left.\frac{\partial\left({ }^{\sigma} \alpha^{0}\right)}{\partial \bar{\theta}}\right|_{\underline{\Omega}}
\end{aligned}
$$

Equation (6.5) can be written as

$$
\begin{aligned}
& {\left[{ }_{d}^{(m)} \bar{\sigma}\right]=\left.\bar{\sigma}^{0}\right|_{\underline{\Omega}}[I]+\sum_{j=1}^{M}{ }^{\sigma} \underline{a}_{j}{ }^{q \sigma} \underline{\sim}^{j}[I]} \\
& +\sum_{i=1}^{N}{ }^{\sigma} b_{i}\left[{ }^{\sigma} G^{i}\right]+\sum_{i=1}^{N} \sum_{j=1}^{M}{ }^{\sigma} \mathcal{c}_{i j}{ }^{q \sigma}{ }_{\sim}{ }^{j}\left[{ }^{\sigma} \underline{\sim}^{i}\right] \\
& +\sum_{i=1}^{N}{ }^{\sigma} d_{i}\left(\bar{\theta}-\bar{\theta}_{\underline{\Omega}}\right)\left[{ }^{\sigma} G^{i}\right]-\left(\alpha_{t m}\right)_{\underline{\Omega}}\left(\bar{\theta}-\bar{\theta}_{\underline{\Omega}}\right)[I]
\end{aligned}
$$

$\left.\bar{\sigma}^{0}\right|_{\Omega}$ is the initial stress in the known configuration $\underline{\Omega}$. Thus, this constitutive theory for $\left[{ }^{(m)}{ }_{d} \bar{\sigma}\right]$ requires determination of $(M+N+(M)(N)+N+1)$ material coefficients. The material coefficients defined in (6.7) are functions of $\bar{\rho}_{\Omega}, \bar{\theta}_{\Omega}$ and $\left({ }^{q \sigma}{ }^{I^{j}}\right)_{\Omega} ; j=1,2, \ldots, M$ in the known configuration $\underline{\Omega}$. This constitutive theory uses integrity, i.e. complete basis. 


\subsection{Constitutive Theory of Orders $(m, n)$ for ${ }^{(0)} \overline{\boldsymbol{q}}$}

Let $\left\{{ }^{q} G^{i}\right\} ; i=1,2, \ldots, \widetilde{N}$ be the combined generators $\left(\right.$ of $\left.{ }^{(0)} \overline{\boldsymbol{q}}\right)$ of the argument tensors $\left[{ }^{(k)} \bar{\sigma}\right] ; k=$ $0,1, \ldots, m-1,\left[{ }^{(j)} \gamma\right] ; j=1,2, \ldots, n$ and $\overline{\boldsymbol{g}}$ that are tensors of rank one. The combined invariants of these argument tensors obviously remain the same as for $\left[{ }^{(m)}{ }_{d} \bar{\sigma}\right]$, i.e. ${ }^{q \sigma} I^{j} ; j=1,2, \ldots, M$. Then we can express ${ }^{(0)} \overline{\boldsymbol{q}}$ as a linear combination of $\left\{{ }^{q} G^{i}\right\} ; i=1,2, \ldots, \widetilde{N}$ in the current configuration.

$$
\left\{{ }^{(0)} \bar{q}\right\}=-\sum_{i=1}^{\tilde{N}}{ }^{q} \alpha^{i}\left\{{ }^{q} G^{i}\right\}
$$

The absence of unit vector in (6.9) as a generator is due to the fact that uniform temperature field does not contribute to ${ }^{(0)} \overline{\boldsymbol{q}}$. The negative sign in (6.9) is because a positive ${ }^{(0)} \overline{\boldsymbol{q}}$ in the direction of the exterior unit normal to the surface of the volume of matter results in heat removal from the volume of matter. The coefficients ${ }^{q} \alpha^{i} ; i=1,2, \ldots, \widetilde{N}$ are functions of $\bar{\rho}, \bar{\theta}$ and ${ }^{q \sigma}{ }_{\sim}^{I^{j}} ; j=1,2, \ldots, M$ in the current configuration. To determine the material coefficients from ${ }^{q} \alpha^{i} ; i=1,2, \ldots, \tilde{N}$ (in the current configuration) in (6.9), we consider Taylor series expansion of each ${ }^{q} \alpha^{i} ; i=1,2, \ldots, \widetilde{N}$ about a known configuration $\underline{\Omega}$ in $\bar{\theta}$ and ${ }^{q \sigma}{ }_{\sim}^{I^{j}} ; j=1,2, \ldots, M$ and retain only up to linear terms in $\theta$ and the invariants.

$$
\begin{aligned}
{ }^{q} \alpha^{i}= & \left.{ }^{q} \alpha^{i}\right|_{\underline{\Omega}}+\left.\sum_{j=1}^{M} \frac{\partial\left({ }^{q} \alpha^{i}\right)}{\partial\left({ }^{q \sigma} \underline{\sim}^{j}\right)}\right|_{\underline{\Omega}}\left({ }^{q \sigma} \stackrel{\sim}{I}^{j}-\left({ }^{q \sigma}{ }_{\sim}^{I^{j}}\right) \underline{\Omega}\right) \\
& +\left.\frac{\partial\left({ }^{q} \alpha^{i}\right)}{\partial \bar{\theta}}\right|_{\underline{\Omega}}\left(\bar{\theta}-\bar{\theta}_{\underline{\Omega}}\right) ; i=1,2, \ldots, \widetilde{N}
\end{aligned}
$$

$$
\left.{ }^{q} \alpha^{i}\right|_{\underline{\Omega}},\left.\frac{\partial\left({ }^{q} \alpha^{i}\right)}{\partial\left(q^{q \sigma} \widetilde{I}^{j}\right)}\right|_{\underline{\Omega}} ; j=1,2, \ldots, M \text { and }\left.\frac{\partial\left({ }^{q} \alpha^{i}\right)}{\partial \bar{\theta}}\right|_{\underline{\Omega}} ; i=
$$
$1,2, \ldots, \widetilde{N}$ are functions of $\bar{\rho}_{\Omega}, \bar{\theta}_{\Omega}$ and $\left({ }^{q \sigma} I_{\Omega}^{j}\right)_{\Omega} ; j=$ $1,2, \ldots, M$ whereas ${ }^{q} \alpha^{i}={ }^{q} \alpha^{i}\left(\bar{\rho}_{\underline{\underline{\Omega}}}, \bar{\theta}_{\underline{\underline{\Omega}}},\left({ }^{q \sigma}{ }_{\sim}^{I^{j}}\right)_{\underline{\underline{\Omega}}} ; j=\right.$ $\left.1,2, \ldots, M, \bar{\theta},{ }^{q \sigma}{ }_{\sim}^{I^{j}} ; j=1,2, \ldots, M\right) ; i=1,2, \ldots, \tilde{N}$ in (6.10). When (6.10) is substituted in (6.9), we obtain the final expression for the most general rate constitutive theory of orders $(m, n)$ for ${ }^{(0)} \overline{\boldsymbol{q}}$ for compressible thermoviscoelastic fluids. Details are presented in the following. Substituting from (6.10) into (6.9)

$$
\begin{aligned}
\left\{{ }^{(0)} \bar{q}\right\}= & -\sum_{i=1}^{\tilde{N}}\left(\left.{ }^{q} \alpha^{i}\right|_{\underline{\Omega}}+\left.\sum_{j=1}^{M} \frac{\partial\left({ }^{q} \alpha^{i}\right)}{\partial\left({ }^{q \sigma} I^{j}\right)}\right|_{\underline{\Omega}}\left({ }^{q \sigma}{ }_{\sim}^{j}-\left({ }^{q \sigma} \underline{I}^{j}\right)_{\underline{\Omega}}\right)\right. \\
& \left.+\left.\frac{\partial\left({ }^{q} \alpha^{i}\right)}{\partial \bar{\theta}}\right|_{\underline{\Omega}}\left(\bar{\theta}-\bar{\theta}_{\underline{\Omega}}\right)\right)\left\{{ }^{q} G^{i}\right\}
\end{aligned}
$$

Collecting coefficients (only those defined in the known configuration $\underline{\Omega}$ ) of $\left\{{ }^{q} G^{i}\right\},{ }^{q \sigma} I^{j}\left\{{ }^{q} G^{i}\right\}$ and $(\bar{\theta}-$ $\left.\bar{\theta}_{\underline{\Omega}}\right)\left\{{ }^{q} G^{i}\right\}$ in (6.11) and defining the following material co- efficients.

$$
\begin{aligned}
& { }_{\underline{q}_{i}}=\left.{ }^{q} \alpha^{i}\right|_{\underline{\Omega}}-\left.\sum_{j=1}^{M} \frac{\partial\left({ }^{q} \alpha^{i}\right)}{\partial\left({ }^{q \sigma} \underline{\sim}^{j}\right)}\right|_{\underline{\Omega}} ; i=1,2, \ldots, \widetilde{N} \\
& { }^{q} \mathcal{C}_{i j}=\left.\frac{\partial\left({ }^{q} \alpha^{i}\right)}{\partial\left({ }^{q \sigma} I^{j}\right)}\right|_{\underline{\Omega}} ; i=1,2, \ldots, \widetilde{N} ; j=1,2, \ldots, M \\
& { }^{q} \mathbb{d}_{i}=\left.\frac{\partial\left({ }^{q} \alpha^{i}\right)}{\partial \bar{\theta}}\right|_{\underline{\Omega}} ; i=1,2, \ldots, \widetilde{N}
\end{aligned}
$$

Equation (6.11) can be written as

$$
\begin{aligned}
\left\{{ }^{(0)} \bar{q}\right\}= & -\sum_{i=1}^{\tilde{N}} b_{i}\left\{{ }^{q} G^{i}\right\}-\sum_{i=1}^{\tilde{N}} \sum_{j=1}^{M}{ }^{q} \mathcal{C}_{i j}{ }^{q \sigma}{ }_{\sim}{ }^{j}\left\{{ }^{q} G^{i}\right\} \\
& -\sum_{i=1}^{\tilde{N}} d_{i}\left(\bar{\theta}-\bar{\theta}_{\underline{\Omega}}\right)\left\{{ }^{q} G^{i}\right\}
\end{aligned}
$$

Thus, this constitutive theory for ${ }^{(0)} \overline{\boldsymbol{q}}$ requires $(\widetilde{N}+$ $(\widetilde{N})(\widetilde{M})+\widetilde{N})$ material coefficients. The material coefficients defined in (6.12) are functions of $\bar{\rho}_{\underline{\Omega}}, \bar{\theta}_{\underline{\Omega}}$ and $\left({ }^{q \sigma}{ }_{\widetilde{I}}^{j}\right)_{\underline{\Omega}}$ $; j=1,2, \ldots, M$ in the known configuration $\underline{\Omega}$. Just like theory for the deviatoric Cauchy stress tensor, this constitutive theory is also based on integrity, i.e. complete basis.

\subsection{Remarks}

1. In sections $6.1-6.2$ we have presented rate constitutive theories of orders $(m, n)$ for the deviatoric Cauchy stress tensor and heat vector using $\left[{ }^{(m)}{ }_{d} \bar{\sigma}\right]$ and ${ }^{(0)} \overline{\boldsymbol{q}}$ as dependent variables with $\left[{ }^{(k)}{ }_{d} \bar{\sigma}\right]$ $; k=0,1, \ldots, m-1$ stress rate tensors and $\left[{ }^{(j)} \gamma\right]$; $j=1,2, \ldots, n$ strain rate tensors as their argument tensors, in addition to $\bar{\rho}, \bar{\theta}$ and $\overline{\boldsymbol{g}}$. Hence, these developments are independent of the basis.

2. By replacing $\left[{ }^{(k)} \bar{d}\right] ; k=0,1, \ldots, m,{ }^{(0)} \overline{\boldsymbol{q}}$ and $\left[{ }^{(j)} \gamma\right]$ ; $j=1,2, \ldots, n$ with the appropriate corresponding measures in the chosen basis, we can readily obtain the rate theories of orders $(m, n)$ for the deviatoric Cauchy stress tensor and the heat vector in the basis of choice. More specifically we use the following measures:

Contravariant basis: $\left[{ }_{d} \bar{\sigma}^{(k)}\right], \quad \overline{\boldsymbol{q}}^{(0)}, \quad\left[\gamma^{(j)}\right]$

$\left.\begin{array}{lllll}\text { Covariant basis: } \quad\left[{ }_{d} \bar{\sigma}_{(k)}\right], & \overline{\boldsymbol{q}}_{(0)} & , & {\left[\gamma_{(j)}\right.}\end{array}\right]$

Jaumann basis: $\quad\left[{ }^{(k)} \bar{\sigma}^{J}\right], \quad{ }^{(0)} \overline{\boldsymbol{q}}^{J}, \quad\left[{ }^{(j)} \gamma^{J}\right]$

where $k=0,1, \ldots, m$ and $j=1,2, \ldots, n$.

3. Since the tensor $\overline{\boldsymbol{g}}$ is independent of the choice of basis, the combined generators and the combined invariants used in sections $6.1-6.2$ only need to be redefined using the convected rates $\left[\gamma^{(j)}\right] ; j=1,2, \ldots, n,\left[\gamma_{(j)}\right] ; j=1,2, \ldots, n,\left[{ }^{(j)} \gamma^{J}\right]$ 


\subsection{Constitutive Theory of Orders $(m, n)$ for ${ }^{(0)} \bar{q}$}

Let $\left\{{ }^{q} G^{i}\right\} ; i=1,2, \ldots, \widetilde{N}$ be the combined generators $\left(\right.$ of $\left.{ }^{(0)} \overline{\boldsymbol{q}}\right)$ of the argument tensors $\left[{ }^{(k)} \bar{d}\right] ; k=$ $0,1, \ldots, m-1,\left[{ }^{(j)} \gamma\right] ; j=1,2, \ldots, n$ and $\overline{\boldsymbol{g}}$ that are tensors of rank one. The combined invariants of these argument tensors obviously remain the same as for $\left[{ }^{(m)} \bar{\sigma}\right]$, i.e. ${ }^{q \sigma} I^{j} ; j=1,2, \ldots, M$. Then we can express ${ }^{(0)} \overline{\boldsymbol{q}}$ as a linear combination of $\left\{{ }^{q} G^{i}\right\} ; i=1,2, \ldots, \widetilde{N}$ in the current configuration.

$$
\left\{{ }^{(0)} \bar{q}\right\}=-\sum_{i=1}^{\tilde{N}}{ }^{q} \alpha^{i}\left\{{ }^{q} G^{i}\right\}
$$

The absence of unit vector in (6.9) as a generator is due to the fact that uniform temperature field does not contribute to ${ }^{(0)} \overline{\boldsymbol{q}}$. The negative sign in (6.9) is because a positive ${ }^{(0)} \overline{\boldsymbol{q}}$ in the direction of the exterior unit normal to the surface of the volume of matter results in heat removal from the volume of matter. The coefficients ${ }^{q} \alpha^{i} ; i=1,2, \ldots, \widetilde{N}$ are functions of $\bar{\rho}, \bar{\theta}$ and ${ }^{q \sigma}{ }^{j} ; j=1,2, \ldots, M$ in the current configuration. To determine the material coefficients from ${ }^{q} \alpha^{i} ; i=1,2, \ldots, \tilde{N}$ (in the current configuration) in (6.9), we consider Taylor series expansion of each ${ }^{q} \alpha^{i} ; i=1,2, \ldots, \widetilde{N}$ about a known configuration $\underline{\Omega}$ in $\bar{\theta}$ and ${ }^{q \sigma}{ }_{\widetilde{\theta}}^{I^{j}} ; j=1,2, \ldots, M$ and retain only up to linear terms in $\bar{\theta}$ and the invariants.

$$
\begin{aligned}
{ }^{q} \alpha^{i}=\left.{ }^{q} \alpha^{i}\right|_{\underline{\Omega}}+\left.\sum_{j=1}^{M} \frac{\partial\left({ }^{q} \alpha^{i}\right)}{\partial\left({ }^{q \sigma} \underline{\sim}^{j}\right)}\right|_{\underline{\Omega}}\left({ }^{q \sigma} \underline{\sim}^{j}-\left({ }^{q \sigma} \underline{\sim}^{j}\right)_{\underline{\Omega}}\right) \\
+\left.\frac{\partial\left({ }^{q} \alpha^{i}\right)}{\partial \bar{\theta}}\right|_{\underline{\Omega}}\left(\bar{\theta}-\bar{\theta}_{\underline{\Omega}}\right) ; i=1,2, \ldots, \widetilde{N}
\end{aligned}
$$

$\left.{ }^{q} \alpha^{i}\right|_{\underline{\Omega}},\left.\frac{\partial\left({ }^{q} \alpha^{i}\right)}{\partial\left({ }^{q \sigma} \underline{I}^{j}\right)}\right|_{\underline{\Omega}} ; j=1,2, \ldots, M$ and $\left.\frac{\partial\left({ }^{q} \alpha^{i}\right)}{\partial \bar{\theta}}\right|_{\underline{\Omega}} ; i=$ $1,2, \ldots, \widetilde{N}$ are functions of $\bar{\rho}_{\underline{\Omega}}, \bar{\theta}_{\Omega}$ and $\left({ }^{q \sigma}{ }^{\sigma}{ }^{j}\right)_{\Omega} ; j=$ $1,2, \ldots, M$ whereas ${ }^{q} \alpha^{i}={ }^{q} \alpha^{i}\left(\overline{\bar{\rho}}_{\underline{\Omega}}, \bar{\theta}_{\underline{\Omega}},\left({ }^{q \sigma}{ }_{\sim}^{I^{j}}\right)_{\underline{\Omega}} ; j=\right.$ $\left.1,2, \ldots, M, \bar{\theta},{ }^{q \sigma} \widetilde{\sim}^{j} ; j=1,2, \ldots, M\right) ; i=1,2, \ldots, \widetilde{N}$ in (6.10). When (6.10) is substituted in (6.9), we obtain the final expression for the most general rate constitutive theory of orders $(m, n)$ for ${ }^{(0)} \overline{\boldsymbol{q}}$ for compressible thermoviscoelastic fluids. Details are presented in the following. Substituting from (6.10) into (6.9)

$$
\begin{aligned}
\left\{{ }^{(0)} \bar{q}\right\}= & -\sum_{i=1}^{\tilde{N}}\left(\left.{ }^{q} \alpha^{i}\right|_{\underline{\Omega}}+\left.\sum_{j=1}^{M} \frac{\partial\left({ }^{q} \alpha^{i}\right)}{\partial\left({ }^{q \sigma} \underline{\sim}^{j}\right)}\right|_{\underline{\Omega}}\left({ }^{q \sigma}{ }_{\sim}^{I^{j}}-\left({ }^{q \sigma} \underline{\sim}^{j}\right) \underline{\underline{\Omega}}\right)\right. \\
& \left.+\left.\frac{\partial\left({ }^{q} \alpha^{i}\right)}{\partial \bar{\theta}}\right|_{\underline{\Omega}}\left(\bar{\theta}-\bar{\theta}_{\underline{\Omega}}\right)\right)\left\{{ }^{q} G^{i}\right\}
\end{aligned}
$$

Collecting coefficients (only those defined in the known configuration $\underline{\Omega})$ of $\left\{{ }^{q} G^{i}\right\},{ }^{q \sigma}{ }_{\sim}^{j}\left\{{ }^{q} G^{i}\right\}$ and $(\bar{\theta}-$ $\left.\bar{\theta}_{\underline{\Omega}}\right)\left\{{ }^{q} G^{i}\right\}$ in (6.11) and defining the following material co- efficients.

$$
\begin{aligned}
& { }_{\underline{b}_{i}}=\left.{ }^{q} \alpha^{i}\right|_{\underline{\Omega}}-\left.\sum_{j=1}^{M} \frac{\partial\left({ }^{q} \alpha^{i}\right)}{\partial\left({ }^{q \sigma} \underline{\sim}^{j}\right)}\right|_{\underline{\Omega}} ; i=1,2, \ldots, \tilde{N} \\
& { }^{q} \mathcal{C}_{i j}=\left.\frac{\partial\left({ }^{q} \alpha^{i}\right)}{\partial\left({ }^{q \sigma} \underline{\sim}^{j}\right)}\right|_{\underline{\Omega}} ; i=1,2, \ldots, \widetilde{N} ; j=1,2, \ldots, M \\
& { }^{q} \mathbb{d}_{i}=\left.\frac{\partial\left({ }^{q} \alpha^{i}\right)}{\partial \bar{\theta}}\right|_{\underline{\Omega}} ; i=1,2, \ldots, \widetilde{N}
\end{aligned}
$$

Equation (6.11) can be written as

$$
\begin{aligned}
\left\{{ }^{(0)} \bar{q}\right\}= & -\sum_{i=1}^{\tilde{N}} b_{i}\left\{{ }^{q} G^{i}\right\}-\sum_{i=1}^{\tilde{N}} \sum_{j=1}^{M}{ }^{q} C_{i j}{ }^{q \sigma} I_{\sim}^{j}\left\{{ }^{q} G^{i}\right\} \\
& -\sum_{i=1}^{\tilde{N}}{ }^{q} d_{i}\left(\bar{\theta}-\bar{\theta}_{\underline{\Omega}}\right)\left\{{ }^{q} G^{i}\right\}
\end{aligned}
$$

Thus, this constitutive theory for ${ }^{(0)} \overline{\boldsymbol{q}}$ requires $(\widetilde{N}+$ $(\widetilde{N})(\widetilde{M})+\widetilde{N})$ material coefficients. The material coefficients defined in (6.12) are functions of $\bar{\rho}_{\underline{\Omega}}, \bar{\theta}_{\underline{\Omega}}$ and $\left({ }^{q \sigma}{ }_{\sim}{ }^{j}\right)_{\underline{\Omega}}$ $; j=1,2, \ldots, M$ in the known configuration $\underline{\Omega}$. Just like theory for the deviatoric Cauchy stress tensor, this constitutive theory is also based on integrity, i.e. complete basis.

\subsection{Remarks}

1. In sections $6.1-6.2$ we have presented rate constitutive theories of orders $(m, n)$ for the deviatoric Cauchy stress tensor and heat vector using $\left[{ }^{(m)} \bar{d} \bar{\sigma}\right]$ and ${ }^{(0)} \overline{\boldsymbol{q}}$ as dependent variables with $\left[{ }^{(k)}{ }_{d} \bar{\sigma}\right]$ $; k=0,1, \ldots, m-1$ stress rate tensors and $\left[{ }^{(j)} \gamma\right]$; $j=1,2, \ldots, n$ strain rate tensors as their argument tensors, in addition to $\bar{\rho}, \bar{\theta}$ and $\overline{\boldsymbol{g}}$. Hence, these developments are independent of the basis.

2. By replacing $\left[{ }^{(k)} \bar{d} \bar{\sigma}\right] ; k=0,1, \ldots, m,{ }^{(0)} \overline{\boldsymbol{q}}$ and $\left[{ }^{(j)} \gamma\right]$ $; j=1,2, \ldots, n$ with the appropriate corresponding measures in the chosen basis, we can readily obtain the rate theories of orders $(m, n)$ for the deviatoric Cauchy stress tensor and the heat vector in the basis of choice. More specifically we use the following measures:

Contravariant basis: $\left[{ }_{d} \bar{\sigma}^{(k)}\right], \quad \overline{\boldsymbol{q}}^{(0)}, \quad\left[\gamma^{(j)}\right]$

$\begin{array}{lllll}\text { Covariant basis: } & {\left[{ }_{d} \bar{\sigma}_{(k)}\right],} & \overline{\boldsymbol{q}}_{(0)} & , & {\left[\gamma_{(j)}\right]}\end{array}$

Jaumann basis: $\quad\left[{ }^{(k)} \bar{\sigma}^{J}\right], \quad{ }^{(0)} \overline{\boldsymbol{q}}^{J}, \quad\left[{ }^{(j)} \gamma^{J}\right]$

where $k=0,1, \ldots, m$ and $j=1,2, \ldots, n$.

3. Since the tensor $\overline{\boldsymbol{g}}$ is independent of the choice of basis, the combined generators and the combined invariants used in sections $6.1-6.2$ only need to be redefined using the convected rates $\left[\gamma^{(j)}\right] ; j=1,2, \ldots, n,\left[\gamma_{(j)}\right] ; j=1,2, \ldots, n,\left[{ }^{(j)} \gamma^{J}\right]$ 
in which the coefficients ${ }^{q} \alpha^{i} ; i=1,2, \ldots, 7$ are functions of $\bar{\rho}, \bar{\theta}$ and ${ }^{q \sigma}{ }_{\sim}^{I^{j}} ; j=1,2, \ldots, 16$ in the current configuration. To determine the material coefficients from ${ }^{q} \alpha^{i} ; i=1,2, \ldots, 7$ (in the current configuration) in (7.5), we consider Taylor series expansion of each ${ }^{q} \alpha^{i}$ $; i=1,2, \ldots, 7$ about the known configuration $\underline{\Omega}$ in $\bar{\theta}$ and ${ }^{q \sigma}{\underset{\sim}{I}}^{j} ; j=1,2, \ldots, 16$ and retain only up to linear terms in $\bar{\theta}$ and the invariants.

$$
\begin{gathered}
{ }^{q} \alpha^{i}=\left.{ }^{q} \alpha^{i}\right|_{\underline{\Omega}}+\left.\sum_{j=1}^{16} \frac{\partial\left({ }^{q} \alpha^{i}\right)}{\partial\left({ }^{q \sigma} \underline{\sim}^{j}\right)}\right|_{\underline{\Omega}}\left({ }^{q \sigma} \underline{\sim}^{j}-\left({ }^{q \sigma} \underline{\sim}^{j}\right)_{\underline{\Omega}}\right) \\
+\left.\frac{\partial\left({ }^{q} \alpha^{i}\right)}{\partial \bar{\theta}}\right|_{\underline{\Omega}}\left(\bar{\theta}-\bar{\theta}_{\underline{\Omega}}\right) ; i=1,2, \ldots, 7
\end{gathered}
$$

We note that $\left.{ }^{q} \alpha^{i}\right|_{\underline{\Omega}},\left.\frac{\partial\left({ }^{q} \alpha^{i}\right)}{\partial\left({ }^{q \sigma} \underline{\sim}^{j}\right)}\right|_{\underline{\Omega}} ; j=1,2, \ldots, 16$ and $\left.\frac{\partial\left({ }^{q} \alpha^{i}\right)}{\partial \bar{\theta}}\right|_{\Omega} ; i=1,2, \ldots, 7$ are functions of $\bar{\rho}_{\underline{\Omega}}, \bar{\theta}_{\underline{\Omega}}$ and $\left({ }^{q \sigma} \underline{\sim}^{j}\right)_{\underline{\Omega}} ; j=1,2, \ldots, 16$ but ${ }^{q} \alpha^{i}={ }^{q} \alpha^{i}\left(\bar{\rho}_{\underline{\Omega}}, \bar{\theta}_{\underline{\Omega}},\left({ }^{q \sigma} \underline{\sim}^{j}\right)_{\underline{\Omega}} ; j=\right.$ $\left.1,2, \ldots, 16, \bar{\theta},{ }^{q \sigma}{ }_{\sim}{ }^{j} ; j=1,2, \ldots, 16\right) ; 1,2, \ldots, 7$ in (7.6).

By substituting (7.6) in (7.5) and following the details presented in section 6.2, we obtain the general form of the rate constitutive theory for the heat vector ${ }^{(0)} \overline{\boldsymbol{q}}$ of orders $1(m=1, n=1)$ for compressible thermoviscoelastic fluids. This constitutive theory contains $(16+7+(7)(16)+7+1)$ material coefficients. As in the case of $\left[{ }^{(1)} \bar{\sigma}\right]$, this theory also contains too many ma- terial coefficients. The simplifications of this theory are considered in the following sections.

\subsection{Further Assumptions and Simplifications}

In order to derive Giesekus model from the rate constitutive theory of orders $m=1$ and $n=1$ for the deviatoric Cauchy stress tensor and the constitutive theory for the heat vector, we make further assumptions in (7.1) and (7.2). We assume that $\left[{ }^{(1)}{ }_{d} \bar{\sigma}\right]$ does not depend on $\overline{\boldsymbol{g}}$ hence we can eliminate $\overline{\boldsymbol{g}}$ as an argument tensor from (7.1). We also assume that the heat vector only depend upon $\overline{\boldsymbol{g}}, \bar{\rho}$ and $\bar{\theta}$ in (7.2), thus we can eliminate $\left[{ }^{(0)}{ }_{d} \bar{\sigma}\right]$ and $\left.{ }^{(1)} \gamma\right]$ from the arguments of the heat vector in (7.2).

$$
\begin{aligned}
& {\left[{ }^{(1)} \bar{d} \bar{\sigma}\right]=\left[{ }^{(1)}{ }_{d} \bar{\sigma}\left(\bar{\rho},\left[{ }^{(0)}{ }_{d} \bar{\sigma}\right],\left[{ }^{(1)} \gamma\right], \bar{\theta}\right)\right]} \\
& { }^{(0)} \overline{\boldsymbol{q}}={ }^{(0)} \overline{\boldsymbol{q}}(\bar{\rho}, \bar{\theta}, \overline{\boldsymbol{g}})
\end{aligned}
$$

\subsubsection{Constitutive Theory for $\left[{ }^{(1)}{ }_{d} \bar{\sigma}\right]$}

The development of the constitutive theory in this case requires: (1) combined generators of $\left[{ }_{d}^{(0)} \bar{\sigma}\right]$ and $\left.{ }^{(1)} \gamma\right]$ (both symmetric tensors of rank two) that are also symmetric tensors of rank two due to the fact that $\left[{ }^{(1)}{ }_{d} \bar{\sigma}\right]$ is a symmetric tensor of rank two (2) combined invariants of the tensors $\left[{ }^{(0)}{ }_{d} \bar{\sigma}\right]$ and $\left[{ }^{(1)} \gamma\right]$. The combined generators and the invariants are listed in Tables $\mathbf{1}$ and $\mathbf{2}$.

Table 1: Combined generators for $\left[{ }^{(1)}{ }_{d} \bar{\sigma}\right]: \boldsymbol{m}=\mathbf{1}, \boldsymbol{n}=\mathbf{1}$; first order rate theory

Arguments

(1) none

(2) one at a time ( including (1) )
$\left[{ }^{(0)} \bar{\sigma} \bar{\sigma}\right]$
$\left[{ }^{\sigma} G^{1}\right]=\left[{ }^{(0)}{ }_{d} \bar{\sigma}\right] \quad ; \quad\left[{ }^{\sigma} G^{2}\right]=\left[{ }^{(0)}{ }_{d} \bar{\sigma}\right]^{2}$
$\left.{ }^{(1)} \gamma\right]$
$\left[{ }^{\sigma} G^{3}\right]=\left[{ }^{(1)} \gamma\right] \quad ; \quad\left[{ }^{\sigma} G^{4}\right]=\left[{ }^{(1)} \gamma\right]^{2}$

Generators

(3) two at a time (including (1) and (2) )

$$
\begin{aligned}
{\left.\left[{ }^{(0)}{ }_{d} \bar{\sigma}\right],\left[{ }^{(1)} \gamma\right] G^{5}\right] } & =\left[{ }^{(0)}{ }_{d} \bar{\sigma}\right]\left[{ }^{(1)} \gamma\right]+\left[{ }^{(1)} \gamma\right]\left[{ }^{(0)}{ }_{d} \bar{\sigma}\right] \\
{\left[{ }^{\sigma} G^{6}\right] } & =\left[{ }^{(0)}{ }_{d} \bar{\sigma}\right]^{2}\left[{ }^{(1)} \gamma\right]+\left[{ }^{(1)} \gamma\right]\left[{ }^{(0)} \bar{d}\right]^{2} \\
{\left[{ }^{\sigma} G^{7}\right] } & =\left[{ }^{(0)}{ }_{d} \bar{\sigma}\right]\left[{ }^{(1)} \gamma\right]^{2}+\left[{ }^{(1)} \gamma\right]^{2}\left[{ }^{(0)}{ }_{d} \bar{\sigma}\right]
\end{aligned}
$$




\section{Remarks:}

1. We note that the invariants listed in table 2 under (2) marked (a) need not be included due to the fact that

$$
\begin{gathered}
\operatorname{tr}\left(\left[{ }^{(0)} \bar{d} \bar{\sigma}\right]\left[{ }^{(1)} \gamma\right]+\left[{ }^{(1)} \gamma\right]\left[{ }^{(0)} \bar{\sigma} \bar{\sigma}\right]\right) \\
+\operatorname{tr}\left(\left[{ }^{(0)} \bar{d} \bar{\sigma}\right]\left[{ }^{(1)} \gamma\right]-\left[{ }^{(1)} \gamma\right]\left[{ }^{(0)}{ }_{d} \bar{\sigma}\right]\right)= \\
2 \operatorname{tr}\left(\left[{ }^{(0)} \bar{\sigma}\right]\left[{ }^{(1)} \gamma\right]\right)
\end{gathered}
$$

which is same as ${ }^{\sigma} I^{7}$ (except for the factor 2 which is of no consequence).

2. In many published works (a) are also included in the list of invariants in addition to ${ }^{q \sigma}{ }_{\sim}{ }^{7}$ which is redundant.

Using the generators in Table 1 we can express $\left[{ }^{(1)} \bar{\sigma}\right]$ as a linear combination of $[I]$ and the combined generators $\left[{ }^{\sigma} G^{i}\right] ; i=1,2, \ldots, 7$. Thus, we can write the following in the current configuration.

$$
\left[{ }^{(1)} \bar{\sigma}\right]={ }^{\sigma} \alpha^{0}[I]+\sum_{i=1}^{7}{ }^{\sigma} \alpha^{i}\left[{ }^{\sigma} G^{i}\right]
$$

The coefficients ${ }^{\sigma} \alpha^{i} ; i=0,1, \ldots, 7$ are functions of the combined invariants ${ }^{\sigma} I^{j} ; j=1,2, \ldots, 10$, density $\bar{\rho}$ and temperature $\bar{\theta}$. The material coefficients are determined from ${ }^{\sigma} \alpha^{i} ; i=0,1, \ldots, 7$ by using Taylor series expansion for each ${ }^{\sigma} \alpha^{i}$ about the known configuration $\underline{\Omega}$ and only retaining up to linear terms in the combined invariants ${ }^{\sigma} I^{j}$ and temperature $\bar{\theta}$.

$$
\begin{aligned}
\sigma_{\alpha^{i}}=\left.^{\sigma} \alpha^{i}\right|_{\underline{\Omega}}+\left.\sum_{j=1}^{10} \frac{\partial\left({ }^{\sigma} \alpha^{i}\right)}{\partial\left({ }^{\sigma} \underline{\sim}^{j}\right)}\right|_{\underline{\Omega}}\left({ }^{\sigma} \underline{\sim}^{j}-\left({ }^{\sigma} \underline{\sim}^{j}\right)_{\underline{\Omega}}\right) \\
+\left.\frac{\partial\left({ }^{\sigma} \alpha^{i}\right)}{\partial \bar{\theta}}\right|_{\underline{\Omega}}\left(\bar{\theta}-\bar{\theta}_{\underline{\Omega}}\right) ; i=0,1, \ldots, 7
\end{aligned}
$$

We note that $\left.{ }^{\sigma} \alpha^{i}\right|_{\underline{\Omega}},\left.\frac{\partial\left(\sigma^{\sigma} \alpha^{i}\right)}{\partial\left(\sigma^{\sigma}{ }^{j}\right)}\right|_{\underline{\Omega}} ; j=1,2, \ldots, 10$ and $\left.\frac{\partial\left({ }^{\sigma} \alpha^{i}\right)}{\partial \bar{\theta}}\right|_{\Omega} ; i=0,1, \ldots, 7$ are functions of $\bar{\rho}_{\underline{\Omega}}, \bar{\theta}_{\underline{\Omega}}$ and $\left({ }^{\sigma} \underline{\Omega}^{j}\right)_{\underline{\Omega}}$ $; j=1,2, \ldots, 10$ but ${ }^{\sigma} \alpha^{i}={ }^{\sigma} \alpha^{i}\left(\bar{\rho}_{\underline{\Omega}}, \bar{\theta}_{\underline{\Omega}},\left({ }^{\sigma} \underline{\sim}^{j}\right)_{\underline{\Omega}} ; j=\right.$ $\left.1,2, \ldots, 10, \bar{\theta},{ }^{\sigma} \stackrel{\sim}{ }^{j} ; j=1,2, \ldots, 10\right) ; i=0,1, \ldots, 7$ in (7.10).

By substituting (7.10) in (7.9), we obtain the rate constitutive theory for $\left[{ }^{(1)} \bar{\sigma}\right]$ based on the argument tensors in (7.7). We note that this expression for $\left[{ }^{(1)} \bar{d}\right]$ contains all the combined generators and invariants of the argument tensors listed in Tables $\mathbf{1}$ and $\mathbf{2}$ and is a non-linear relationship in $\left[{ }^{(0)} \bar{\sigma} \bar{\sigma}\right]$ and $\left[{ }^{(1)} \gamma\right]$ but it is a first order rate theory $(m=1$ and $n=1)$.

This rate theory still contains many material coefficients but its further simplifications form the basis for Giesekus constitutive model.

Table 2: Combined invariants for $\left[{ }^{(1)} \bar{\sigma}\right]: m=1, n=1$; first order rate theory

Arguments Invariants

(1) one at a time
$\left[{ }^{(0)} \bar{\sigma}\right]$
${ }^{\sigma}{ }_{\sim}^{1}=\operatorname{tr}\left(\left[{ }^{(0)} \bar{d}\right]\right) \quad ; \quad{ }_{\sim}^{\sigma} I^{2}=\operatorname{tr}\left(\left[{ }^{(0)}{ }_{d} \bar{\sigma}\right]^{2}\right)$
${ }^{\sigma} I^{3}=\operatorname{tr}\left(\left[{ }^{(0)} \bar{\sigma}\right]^{3}\right)$
$\left[{ }^{(1)} \gamma\right]$
${ }^{\sigma} I^{4}=\operatorname{tr}\left(\left[{ }^{(1)} \gamma\right]\right) \quad ; \quad{ }^{\sigma} I^{5}=\operatorname{tr}\left(\left[{ }^{(1)} \gamma\right]^{2}\right)$
${ }_{\sim}^{\sigma} I^{6}=\operatorname{tr}\left(\left[{ }^{(1)} \gamma\right]^{3}\right)$

(2) two at a time ( including (1) )

$$
\begin{aligned}
& {\left[{ }^{(0)} \bar{d}\right], \quad\left[{ }^{(1)} \gamma\right] \quad{ }_{\sim}^{\sigma} I^{7}=\operatorname{tr}\left(\left[{ }^{(0)} \bar{d} \bar{\sigma}\right]\left[{ }^{(1)} \gamma\right]\right) ;{ }^{\sigma} I^{8}=\operatorname{tr}\left(\left[{ }^{(0)} \bar{d} \bar{\sigma}\right]^{2}\left[{ }^{(1)} \gamma\right]\right)} \\
& { }_{\sim} I^{9}=\operatorname{tr}\left(\left[{ }^{(0)} \bar{d} \bar{\sigma}\right]\left[{ }^{(1)} \gamma\right]^{2}\right) ;{ }_{\sim} I^{10}=\operatorname{tr}\left(\left[{ }^{(0)}{ }_{d} \bar{\sigma}\right]^{2}\left[{ }^{(1)} \gamma\right]^{2}\right) \\
& \text { (a) } \begin{aligned}
{ }^{\sigma} I & =\operatorname{tr}\left(\left[{ }^{(0)}{ }_{d} \bar{\sigma}\right]\left[\left[^{(1)} \gamma\right]+\left[{ }^{(1)} \gamma\right]\left[{ }^{(0)} \bar{\sigma} \bar{\sigma}\right]\right)\right. \\
& =\operatorname{tr}\left(\left[{ }^{(0)}{ }_{d} \bar{\sigma}\right]\left[{ }^{(1)} \gamma\right]-\left[{ }^{(1)} \gamma\right][(0) \bar{\sigma}]\right)
\end{aligned}
\end{aligned}
$$




\subsubsection{Constitutive Theory for ${ }^{(0)} \bar{q}$}

Consider (7.8) for the heat vector ${ }^{(0)} \overline{\boldsymbol{q}}$. In this case, the generators of ${ }^{(0)} \overline{\boldsymbol{q}}$ that are tensors of rank one are given by $\overline{\boldsymbol{g}}$ only. Also, in this case the only invariant is ${ }^{q} \underset{\sim}{I}=\overline{\boldsymbol{g}} \cdot \overline{\boldsymbol{g}}$. Thus, we can write the following in the current configuration.

$$
{ }^{(0)} \overline{\boldsymbol{q}}=-{ }^{q} \alpha \overline{\boldsymbol{g}}
$$

The coefficient ${ }^{q} \alpha$ in (7.11) is a function of $\bar{\rho}, \bar{\theta}$ and ${ }^{q} I$ in the current configuration, i.e. $\bar{\rho}, \bar{\theta}$ and ${ }^{q} I$. To determine the material coefficient from ${ }^{q} \alpha$ in (7.11) related to the current configuration, we consider Taylor series expansion of ${ }^{q} \alpha$ about a known configuration $\underline{\Omega}$ in $\bar{\theta}$ and ${ }^{q} \underline{I}$ and retain only up to linear terms in $\bar{\theta}$ and the invariant.

${ }^{q} \alpha=\left.{ }^{q} \alpha\right|_{\underline{\Omega}}+\left.\frac{\partial\left({ }^{q} \alpha\right)}{\partial\left({ }^{q} I\right)}\right|_{\underline{\Omega}}\left(\overline{\boldsymbol{g}} \cdot \overline{\boldsymbol{g}}-(\overline{\boldsymbol{g}} \cdot \overline{\boldsymbol{g}})_{\underline{\Omega}}\right)+\left.\frac{\partial\left({ }^{q} \alpha\right)}{\partial \bar{\theta}}\right|_{\underline{\underline{\Omega}}}\left(\bar{\theta}-\bar{\theta}_{\underline{\Omega}}\right)$

$\left.{ }^{q} \alpha\right|_{\underline{\Omega}},\left.\frac{\partial\left({ }^{q} \alpha\right)}{\partial\left({ }^{q} I\right)}\right|_{\underline{\Omega}}$ and $\left.\frac{\left.\partial{ }^{(q} \alpha\right)}{\partial \bar{\theta}}\right|_{\underline{\Omega}}$ are functions of $\bar{\rho}_{\underline{\underline{\Omega}}}, \bar{\theta}_{\underline{\underline{\Omega}}}$ and $(\overline{\boldsymbol{g}} \cdot \overline{\boldsymbol{g}})_{\Omega}$, however ${ }^{q} \alpha={ }^{q} \alpha\left(\bar{\rho}_{\Omega}, \bar{\theta}_{\Omega},(\overline{\boldsymbol{g}} \cdot \overline{\boldsymbol{g}})_{\Omega}, \bar{\theta}, \overline{\boldsymbol{g}} \cdot \overline{\boldsymbol{g}}\right)$ in (7.12). Substituting from (7.12) into (7.11)

${ }^{(0)} \overline{\boldsymbol{q}}=-\left.{ }^{q} \alpha\right|_{\underline{\Omega}} \overline{\boldsymbol{g}}-\left.\frac{\partial\left({ }^{q} \alpha\right)}{\partial\left({ }^{q} I\right)}\right|_{\underline{\Omega}}\left(\overline{\boldsymbol{g}} \cdot \overline{\boldsymbol{g}}-(\overline{\boldsymbol{g}} \cdot \overline{\boldsymbol{g}})_{\underline{\Omega}}\right) \overline{\boldsymbol{g}}-\left.\frac{\partial\left({ }^{q} \alpha\right)}{\partial \bar{\theta}}\right|_{\underline{\Omega}}\left(\bar{\theta}-\bar{\theta}_{\underline{\Omega}}\right) \overline{\boldsymbol{g}}$

We note that if there is a uniform temperature change between the known and current configurations, then $\overline{\boldsymbol{g}}=$ 0 and hence ${ }^{(0)} \overline{\boldsymbol{q}}$ must be zero. This condition is satisfied by (7.13). If we define $k_{\underline{\underline{\Omega}}}=\left.{ }^{q} \alpha\right|_{\underline{\Omega}},{ }^{1} k_{\underline{\underline{\Omega}}}=\left.\frac{\partial\left({ }^{q} \alpha\right)}{\partial\left({ }^{q} I\right)}\right|_{\underline{\Omega}}$ and ${ }^{2} k_{\underline{\Omega}}=\left.\frac{\partial\left({ }^{q} \alpha\right)}{\partial \bar{\theta}}\right|_{\underline{\Omega}}$ then

$$
{ }^{(0)} \overline{\boldsymbol{q}}=-k_{\underline{\underline{\Omega}}} \overline{\boldsymbol{g}}-{ }^{1} k_{\underline{\underline{\Omega}}}\left(\overline{\boldsymbol{g}} \cdot \overline{\boldsymbol{g}}-(\overline{\boldsymbol{g}} \cdot \overline{\boldsymbol{g}})_{\underline{\Omega}}\right) \overline{\boldsymbol{g}}-{ }^{2} k_{\underline{\underline{\Omega}}}\left(\bar{\theta}-\bar{\theta}_{\underline{\underline{\Omega}}}\right) \overline{\boldsymbol{g}}
$$

We note that $k_{\Omega}=k\left(\bar{\rho}_{\Omega}, \bar{\theta}_{\Omega},(\overline{\boldsymbol{g}} \cdot \overline{\boldsymbol{g}})_{\underline{\Omega}}\right),{ }^{1} k_{\underline{\Omega}}=$ ${ }^{1} k\left(\bar{\rho}_{\underline{\Omega}}, \bar{\theta}_{\underline{\Omega}},(\overline{\boldsymbol{g}} \cdot \overline{\boldsymbol{g}})_{\underline{\Omega}}\right)$ and ${ }^{2} k_{\underline{\underline{\Omega}}}={ }^{\overline{2}} k\left(\bar{\rho}_{\underline{\underline{\Omega}}}, \bar{\theta}_{\underline{\underline{\Omega}}},(\overline{\boldsymbol{g}} \cdot \overline{\overline{\boldsymbol{g}}})_{\underline{\Omega}}\right)$. (7.14) is the most general form of the constitutive equation for the heat vector ${ }^{(0)} \overline{\boldsymbol{q}}$ based on (7.8). If we neglect infinitesimals of order two and higher in the components of $\overline{\boldsymbol{g}}$ as well as the product of $\left(\bar{\theta}-\bar{\theta}_{\underline{\Omega}}\right)$ with $\overline{\boldsymbol{g}}$, then (7.14) reduces to

$$
\begin{aligned}
& { }^{(0)} \overline{\boldsymbol{q}}=-k_{\underline{\Omega}} \overline{\boldsymbol{g}} \\
& \text { or } \quad\left\{{ }^{(0)} \bar{q}\right\}=-k_{\underline{\underline{\Omega}}}\{\bar{g}\}=-k_{\underline{\underline{\Omega}}}[I]\{\bar{g}\}=-[K]\{\bar{g}\}
\end{aligned}
$$

in which $k$ is thermal conductivity and $[K]$ is the diagonal thermal conductivity matrix. We note that

$$
k_{\underline{\underline{\Omega}}}=k\left(\bar{\rho}_{\underline{\underline{\Omega}}}, \bar{\theta}_{\underline{\Omega}},(\overline{\boldsymbol{g}} \cdot \overline{\boldsymbol{g}})_{\underline{\Omega}}\right)
$$

Equations (7.16) is the standard Fourier heat conduction law with variable thermal conductivity. Based on 7.17 , the thermal conductivity can be a function of density, temperature and the first invariant of $\overline{\boldsymbol{g}}$, i.e. $\overline{\boldsymbol{g}} \cdot \overline{\boldsymbol{g}}$. Thus, we can use experimental and/or empirical data for thermal conductivity as a function of density, temperature and $\overline{\boldsymbol{g}} \cdot \overline{\boldsymbol{g}}$ during the evolution, keeping in mind that
7.17 only holds for the known configuration $\underline{\Omega}$ where as ${ }^{(0)} \overline{\boldsymbol{q}}$ and $\overline{\boldsymbol{g}}$ in (7.16) are in the current configuration. This is obviously a consequence of Taylor series expansion of ${ }^{(0)} \overline{\boldsymbol{q}}$ about the known configuration $\underline{\Omega}$. Power law, Sutherland law $[2,16]$ are examples of temperature dependent thermal conductivities.

In currently published works $[2,16]$ the thermal conductivity is generally expressed as a function of the unknown deformation or state in the current configuration, i.e. instead of $k_{\Omega}$ defined by 7.17 , it is replaced by

$$
k=k(\bar{\rho}, \bar{\theta}, \overline{\boldsymbol{g}} \cdot \overline{\boldsymbol{g}})
$$

This obviously makes $k$ a function of unknown $\bar{\rho}, \bar{\theta}$ and $\overline{\boldsymbol{g}} \cdot \overline{\boldsymbol{g}}$ in the current configuration. When the current and known configurations are in close proximity of each other in terms of deformation field, replacing $k$ in (7.16) using 7.18 may be justified, but it is not supported by the derivation of the constitutive theory presented here.

We note that this constitutive theory $((7.14)$ and (7.16)) for ${ }^{(0)} \overline{\boldsymbol{q}}$ is independent of the basis, i.e.

$$
{ }^{(0)} \overline{\boldsymbol{q}}=\overline{\boldsymbol{q}}^{(0)}=\overline{\boldsymbol{q}}_{(0)}={ }^{(0)} \overline{\boldsymbol{q}}^{J}=\overline{\boldsymbol{q}}
$$

\subsection{Giesekus Constitutive Model for Deviatoric Cauchy Stress Tensor}

The general derivation presented for the constitutive theory of order one $(m=1, n=1)$ for deviatoric Cauchy stress in section 7.1 forms the basis for deriving the Giesekus constitutive model used for polymer melts (dense polymers). Giesekus constitutive model is a non-linear viscoelastic model. In the derivation of the Giesekus constitutive model, we begin by considering the combined generators of $\left[{ }^{(0)} \bar{\sigma}\right]$ and $\left[{ }^{(1)} \gamma\right]$ only up to quadratics. Therefore $(7.9)$ reduces

$$
\begin{aligned}
{\left[{ }^{(1)}{ }_{d} \bar{\sigma}\right]=} & { }^{\sigma} \alpha^{0}[I]+{ }^{\sigma} \alpha^{1}\left[{ }^{(0)} \bar{\sigma}\right]+{ }^{\sigma} \alpha^{2}\left[{ }^{(1)} \gamma\right] \\
& +{ }^{\sigma} \alpha^{3}\left[{ }^{(0)} \bar{d} \bar{\sigma}\right]{ }^{2}+{ }^{\sigma} \alpha^{4}\left[{ }^{(1)} \gamma\right]{ }^{2} \\
& +{ }^{\sigma} \alpha^{5}\left(\left[{ }^{(0)} \bar{\sigma}\right]\left[{ }^{(1)} \gamma\right]+\left[{ }^{(1)} \gamma\right]\left[{ }^{(0)} \bar{\sigma}\right]\right)
\end{aligned}
$$

We further neglect the last two generators in (7.20)

$$
\left[{ }^{(1)} \bar{\sigma}\right]={ }^{\sigma} \alpha^{0}[I]+{ }^{\sigma} \alpha^{1}\left[{ }_{d}^{(0)} \bar{\sigma}\right]+{ }^{\sigma} \alpha^{2}\left[{ }^{(1)} \gamma\right]+{ }^{\sigma} \alpha^{3}\left[{ }^{(0)} \bar{\sigma}\right]^{2}
$$

The coefficients ${ }^{\sigma} \alpha^{i} ; i=0,1, \ldots, 3$ depend upon $\bar{\rho}, \bar{\theta}$ and the combined invariants of $\left[{ }^{(0)} \bar{\sigma}\right]$ and $\left[{ }^{(1)} \gamma\right]$, i.e. ${ }^{\sigma}{ }^{j}$ $; j=1,2, \ldots, 10$, in the current configuration. These are listed in Table 2. To determine the material coefficients from ${ }^{\sigma} \alpha^{i} ; i=0,1, \ldots, 3$ in (7.21), we consider Taylor series expansions of ${ }^{\sigma} \alpha^{i} ; i=0,1, \ldots, 3$ about the known configuration $\underline{\Omega}$ in $\bar{\theta}$ and ${ }^{\sigma} \underline{\sim}^{j} ; j=1,2, \ldots, 10$ and retain 
only up to linear terms in $\bar{\theta}$ and the invariants.

$$
\begin{aligned}
\sigma^{\sigma} \alpha^{i}= & \left.{ }^{\sigma} \alpha^{i}\right|_{\underline{\Omega}}+\left.\sum_{j=1}^{10} \frac{\partial\left({ }^{\sigma} \alpha^{i}\right)}{\partial\left({ }^{\sigma} \underline{\sim}^{j}\right)}\right|_{\underline{\Omega}}\left({ }^{\sigma} \stackrel{\sim}{I}^{j}-\left({ }^{\sigma} \stackrel{\sim}{I}^{j}\right)_{\underline{\Omega}}\right) \\
& +\left.\frac{\partial\left({ }^{\sigma} \alpha^{i}\right)}{\partial \bar{\theta}}\right|_{\underline{\Omega}}\left(\bar{\theta}-\bar{\theta}_{\underline{\Omega}}\right) ; i=0,1, \ldots, 3
\end{aligned}
$$

The coefficients $\left.{ }^{\sigma} \alpha^{i}\right|_{\underline{\Omega}},\left.\frac{\partial\left(\sigma^{\sigma} \alpha^{i}\right)}{\partial\left({ }^{\sigma} \underline{\sim}^{j}\right)}\right|_{\underline{\Omega}} ; j=1,2, \ldots, 10$ and $\left.\frac{\partial\left({ }^{\sigma} \alpha^{i}\right)}{\partial \bar{\theta}}\right|_{\underline{\Omega}} ; i=0,1, \ldots, 3$ are functions of $\bar{\rho}_{\underline{\Omega}}, \bar{\theta}_{\underline{\Omega}}$ and $\left({ }^{\sigma} I^{j}\right)_{\underline{\Omega}}$ $; j=1,2, \ldots, 10$, however ${ }^{\sigma} \alpha^{i}={ }^{\sigma} \alpha^{i}\left(\bar{\rho}_{\underline{\Omega}}, \bar{\theta}_{\underline{\Omega}},\left({ }^{\sigma}{ }_{\sim}{ }^{j}\right)_{\underline{\Omega}} ; j=\right.$ $\left.1,2, \ldots, 10, \bar{\theta},{ }^{\sigma}{ }_{\sim}^{I^{j}} ; j=1,2, \ldots, 10\right) ; i=0,1, \ldots, 3$ in (7.22). If we let $\sigma_{\alpha}{ }^{i},{ }_{j}=\frac{\partial\left(\sigma \alpha^{i}\right)}{\partial\left(\sigma^{\sigma^{j}}\right)} ; j=1,2, \ldots, 10$, then (7.22) can be written as

$$
\begin{aligned}
\sigma^{\sigma} \alpha^{i}= & \left.\alpha^{i}\right|_{\underline{\Omega}}+\left.\sum_{j=1}^{10}\left({ }^{\sigma} \alpha^{i},{ }_{j}\right)\right|_{\underline{\Omega}}\left({ }^{\sigma} \underline{\sim}^{j}-\left({ }^{\sigma} \underline{\sim}^{j}\right)_{\underline{\Omega}}\right) \\
& +\left.\frac{\partial\left({ }^{\sigma} \alpha^{i}\right)}{\partial \bar{\theta}}\right|_{\underline{\Omega}}\left(\bar{\theta}-\bar{\theta}_{\underline{\Omega}}\right) \quad ; \quad i=0,1, \ldots, 3
\end{aligned}
$$

Substituting from (7.23) in (7.21), we obtain the most general expression for the constitutive theory for $\left[{ }^{(1)} \bar{\sigma}\right]$ based on the choice of generators in (7.21) and invariants ${ }^{\sigma} I^{j} ; j=1,2, \ldots, 10$ listed in Table 2.

$$
\begin{aligned}
{\left[{ }_{d}^{(1)} \bar{\sigma}\right]=} & \left(\left.{ }^{\sigma} \alpha^{0}\right|_{\underline{\Omega}}+\left.\sum_{j=1}^{10}\left({ }^{\sigma} \alpha^{0},{ }_{j}\right)\right|_{\underline{\Omega}}\left({ }^{\sigma} I^{j}-\left({ }^{\sigma} \underline{\sim}^{j}\right)_{\underline{\Omega}}\right)\right. \\
& \left.+\left.\frac{\partial\left({ }^{\sigma} \alpha^{0}\right)}{\partial \bar{\theta}}\right|_{\underline{\Omega}}\left(\bar{\theta}-\bar{\theta}_{\underline{\Omega}}\right)\right)[I]+ \\
& \left(\left.{ }^{\sigma} \alpha^{1}\right|_{\underline{\Omega}}+\left.\sum_{j=1}^{10}\left({ }^{\sigma} \alpha^{1}, j\right)\right|_{\underline{\Omega}}\left({ }^{\sigma} \underline{\sim}^{j}-\left({ }^{\sigma} \underline{\sim}^{j}\right)_{\underline{\Omega}}\right)\right. \\
& \left.+\left.\frac{\partial\left({ }^{\sigma} \alpha^{1}\right)}{\partial \bar{\theta}}\right|_{\underline{\Omega}}\left(\bar{\theta}-\bar{\theta}_{\underline{\Omega}}\right)\right)\left[{ }_{d}^{(0)} \bar{\sigma}\right]+ \\
& \left(\left.{ }^{\sigma} \alpha^{2}\right|_{\underline{\Omega}}+\left.\sum_{j=1}^{10}\left({ }^{\sigma} \alpha^{2},{ }_{j}\right)\right|_{\underline{\Omega}}\left({ }^{\sigma} \underline{I}^{j}-\left({ }^{\sigma} \underline{I}^{j}\right)_{\underline{\Omega}}\right)\right. \\
& \left.+\left.\frac{\partial\left({ }^{\sigma} \alpha^{2}\right)}{\partial \bar{\theta}}\right|_{\underline{\Omega}}\left(\bar{\theta}-\bar{\theta}_{\underline{\Omega}}\right)\right)\left[{ }^{(1)} \gamma\right]+ \\
& \left(\left.{ }^{\sigma} \alpha^{3}\right|_{\underline{\Omega}}+\left.\sum_{j=1}^{10}\left({ }^{\sigma} \alpha^{3}, j\right)\right|_{\underline{\Omega}}\left({ }^{\sigma} I^{j}-\left({ }^{\sigma} I_{\sim}^{j}\right)_{\underline{\Omega}}\right)\right. \\
& \left.+\left.\frac{\partial\left({ }^{\sigma} \alpha^{3}\right)}{\partial \bar{\theta}}\right|_{\underline{\Omega}}\left(\bar{\theta}-\bar{\theta}_{\underline{\Omega}}\right)\right)\left[{ }^{(0)} \bar{\sigma}\right]^{2}
\end{aligned}
$$

\subsubsection{Further Assumptions and Simplifications}

We further make the following assumptions to derive the Giesekus model, which is a non-linear viscoelastic model.

(i) We delete the terms containing products of the generators $\left[{ }^{(1)} \gamma\right],\left[{ }^{(0)} \bar{\sigma}\right]$ and $\left[{ }^{(0)} \bar{\sigma}\right]^{2}$ with the invariants ${ }^{\sigma}{ }_{\sim}^{j} ; j=1,2, \ldots, 10$ in the current configuration. (ii) We also delete the terms containing the products of the generators $\left[{ }^{(1)} \gamma\right],\left[{ }_{d}^{(0)} \bar{\sigma}\right]$ and $\left[{ }_{d}^{(0)} \bar{\sigma}\right]^{2}$ with $\left(\bar{\theta}-\bar{\theta}_{\underline{\Omega}}\right)$ in the current configuration. This gives

$$
\begin{gathered}
{\left[{ }_{d}^{(1)} \bar{\sigma}\right]=\left(\left.{ }^{\sigma} \alpha^{0}\right|_{\underline{\Omega}}+\left.\sum_{j=1}^{10}\left({ }^{\sigma} \alpha^{0},{ }_{j}\right)\right|_{\underline{\Omega}}\left({ }^{\sigma} \underline{I}^{j}-\left({ }^{\sigma} I_{\sim}^{j}\right)_{\underline{\Omega}}\right)\right.} \\
\left.+\left.\frac{\partial\left({ }^{\sigma} \alpha^{0}\right)}{\partial \bar{\theta}}\right|_{\underline{\Omega}}\left(\bar{\theta}-\bar{\theta}_{\underline{\Omega}}\right)\right)[I] \\
+\left(\left.{ }^{\sigma} \alpha^{1}\right|_{\underline{\Omega}}-\left.\sum_{j=1}^{10}\left({ }^{\sigma} \alpha^{1},{ }_{j}\right)\right|_{\underline{\Omega}}\left({ }^{\sigma} \underline{\sim}^{j}\right)_{\underline{\Omega}}\right)\left[{ }_{d}^{(0)} \bar{\sigma}\right] \\
+\left(\left.{ }^{\sigma} \alpha^{2}\right|_{\underline{\Omega}}-\left.\sum_{j=1}^{10}\left({ }^{\sigma} \alpha^{2},{ }_{j}\right)\right|_{\underline{\Omega}}\left({ }^{\sigma}{ }_{\sim}{ }^{j}\right)_{\underline{\Omega}}\right)\left[{ }^{(1)} \gamma\right] \\
+\left(\left.{ }^{\sigma} \alpha^{3}\right|_{\underline{\underline{\Omega}}}-\left.\sum_{j=1}^{10}\left({ }^{\sigma} \alpha^{3},{ }_{j}\right)\right|_{\underline{\Omega}}\left({ }^{\sigma} \underline{\sim}^{j}\right)_{\underline{\Omega}}\right)\left[{ }^{(0)} \bar{\sigma}\right]^{2}
\end{gathered}
$$

We collect terms and define material coefficients and others. Let

$$
\begin{aligned}
& { }^{\sigma} b^{0}=\left.{ }^{\sigma} \alpha^{0}\right|_{\underline{\Omega}}, \quad{ }^{\sigma} b_{j}^{1}=\left.\left({ }^{\sigma} \alpha^{0}, j\right)\right|_{\underline{\Omega}} \\
& { }^{\sigma} b^{2}=\left.{ }^{\sigma} \alpha^{1}\right|_{\underline{\underline{\Omega}}}-\left.\sum_{j=1}^{10}\left({ }^{\sigma} \alpha^{1},{ }_{j}\right)\right|_{\underline{\underline{\Omega}}}\left({ }^{\sigma} \underline{I}^{j}\right)_{\underline{\underline{\Omega}}} \\
& { }^{\sigma} b^{3}=\left.{ }^{\sigma} \alpha^{2}\right|_{\underline{\underline{\Omega}}}-\left.\sum_{j=1}^{10}\left({ }^{\sigma} \alpha^{2},{ }_{j}\right)\right|_{\underline{\underline{\Omega}}}\left({ }^{\sigma}{ }_{\sim}^{j}\right)_{\underline{\Omega}} \\
& { }^{\sigma} b^{4}=\left.{ }^{\sigma} \alpha^{3}\right|_{\underline{\underline{\Omega}}}-\left.\sum_{j=1}^{10}\left({ }^{\sigma} \alpha^{3},{ }_{j}\right)\right|_{\underline{\underline{\Omega}}}\left({ }^{\sigma} \underline{\sim}^{j}\right)_{\underline{\Omega}}
\end{aligned}
$$

Then we can write

$$
\begin{aligned}
& {\left[{ }_{d}^{(1)} \bar{\sigma}\right]={ }^{\sigma} b^{0}[I]+\sum_{j=1}^{10}{ }^{\sigma} b_{j}^{1 \sigma} \stackrel{\sim}{I}^{j}[I]+{ }^{\sigma} b^{2}\left[{ }_{d}^{(0)} \bar{\sigma}\right]+} \\
& { }^{\sigma} b^{3}\left[{ }^{(1)} \gamma\right]+{ }^{\sigma} b^{4}\left[{ }^{(0)} \bar{\sigma}\right]^{2}+\left.\frac{\partial\left({ }^{\sigma} \alpha^{0}\right)}{\partial \bar{\theta}}\right|_{\underline{\Omega}}\left(\bar{\theta}-\bar{\theta}_{\underline{\Omega}}\right)[I]
\end{aligned}
$$

We note that the coefficients ${ }^{\sigma} b^{0},{ }^{\sigma} b_{j}^{1} ; j=$ $1,2, \ldots, 10,{ }^{\sigma} b^{2},{ }^{\sigma} b^{3},{ }^{\sigma} b^{4}$ and $\left.\frac{\partial\left({ }^{\sigma} \alpha^{0}\right)}{\partial \bar{\theta}}\right|_{\underline{\Omega}}$ are defined in the known configuration $\underline{\Omega}$ and hence are functions of $\bar{\rho}_{\underline{\Omega}}, \bar{\theta}_{\underline{\Omega}}$ and $\left({ }^{\sigma} I^{j}\right)_{\underline{\Omega}} ; j=1,2, \ldots, 10$. The constitutive model (7.27) is quite general based on (7.21) and the assumptions (i) and (ii).

(iii) To derive the Giesekus constitutive model, we neglect all invariants in (7.27) except (based on Table 2) ${ }^{\sigma}{ }_{\sim}^{1}=i_{(0)_{d} \bar{\sigma}}=\operatorname{tr}\left(\left[{ }^{(0)} \bar{\sigma}\right]\right)$ and ${ }^{\sigma}{ }_{\sim}{ }^{4}=i_{(1) \gamma}=$ $\operatorname{tr}\left(\left[{ }^{(1)} \gamma\right]\right)$. Thus $(7.27)$ reduces to the following:

$$
\begin{aligned}
{\left[{ }_{d}^{(1)} \bar{\sigma}\right]=} & { }^{\sigma} b^{0}[I]+{ }^{\sigma} b_{1}^{1} \operatorname{tr}\left(\left[{ }_{d}^{(0)} \bar{\sigma}\right]\right)[I]+{ }^{\sigma} b_{4}^{1} \operatorname{tr}\left(\left[{ }^{(1)} \gamma\right]\right)[I] \\
& +{ }^{\sigma} b^{2}\left[{ }^{(0)} \bar{\sigma}\right]+{ }^{\sigma} b^{3}\left[{ }^{(1)} \gamma\right] \\
& +{ }^{\sigma} b^{4}\left[{ }^{(0)} \bar{\sigma}\right]^{2}+\left.\frac{\partial\left({ }^{\sigma} \alpha^{0}\right)}{\partial \bar{\theta}}\right|_{\underline{\Omega}}\left(\bar{\theta}-\bar{\theta}_{\underline{\Omega}}\right)[I]
\end{aligned}
$$


which can be written as

$$
\begin{aligned}
{\left[{ }_{d}^{(0)} \bar{\sigma}\right] } & +\left(-\frac{1}{\sigma b^{2}}\right)\left[{ }_{d}^{(1)} \bar{\sigma}\right]=\left(-\frac{{ }^{\sigma} b^{0}}{\sigma b^{2}}\right)[I] \\
& +\left(-\frac{{ }^{\sigma} b^{3}}{\sigma b^{2}}\right)\left[{ }^{(1)} \gamma\right]+\left(-\frac{{ }^{\sigma} b_{4}^{1}}{\sigma b^{2}}\right) \operatorname{tr}\left(\left[{ }^{(1)} \gamma\right]\right)[I] \\
& +\left(-\frac{{ }^{\sigma} b_{1}^{1}}{\sigma b^{2}}\right) \operatorname{tr}\left(\left[{ }^{(0)} \bar{d}\right]\right)[I]+\left(-\frac{{ }^{\sigma} b^{4}}{\sigma b^{2}}\right)\left[{ }_{d}^{(0)} \bar{\sigma}\right]^{2} \\
& -\left(\left.\frac{\partial\left({ }^{\sigma} \alpha^{0}\right)}{\partial \bar{\theta}}\right|_{\underline{\Omega}} \frac{1}{\sigma b^{2}}\right)\left(\bar{\theta}-\bar{\theta}_{\underline{\Omega}}\right)[I]
\end{aligned}
$$

We introduce mew notations for the material coefficients to conform to the standard notations used in the literature. Let

$$
\begin{aligned}
& \left(-\frac{1}{\sigma b^{2}}\right)=\lambda_{\underline{\Omega}} ; \quad\left(-\frac{{ }^{\sigma} b^{0}}{\sigma b^{2}}\right)=\left.\bar{\sigma}_{0}\right|_{\underline{\Omega}} \\
& \left(-\frac{{ }^{\sigma} b^{3}}{\sigma b^{2}}\right)=2 \eta_{\underline{\Omega}} ; \quad\left(-\frac{{ }^{\sigma} b_{4}^{1}}{\sigma b^{2}}\right)=\kappa_{\underline{\Omega}} \\
& \left(-\frac{{ }^{\sigma} b_{1}^{1}}{\sigma b^{2}}\right)={ }^{1} \kappa_{\underline{\Omega}} ;\left(\left.\frac{\partial\left({ }^{\sigma} \alpha^{0}\right)}{\partial \bar{\theta}}\right|_{\underline{\Omega}} \frac{1}{\sigma b^{2}}\right)=\left(\alpha_{t m}\right)_{\underline{\Omega}}
\end{aligned}
$$

in which they are all functions of $\bar{\rho}_{\Omega}, \bar{\theta}_{\Omega},\left({ }^{\sigma}{ }_{\sim}{ }^{j}\right)_{\Omega} ; j=$ $1,2, \ldots, 10$. Then (7.29) can be written as

$$
\begin{aligned}
{\left[{ }^{(0)}{ }_{d} \bar{\sigma}\right] } & +\lambda_{\underline{\Omega}}\left[{ }^{(1)}{ }_{d} \bar{\sigma}\right]=\left.\bar{\sigma}_{0}\right|_{\underline{\Omega}}[I]+2 \eta_{\underline{\underline{\Omega}}}\left[{ }^{(1)} \gamma\right] \\
& +\kappa_{\underline{\underline{\Omega}}} \operatorname{tr}\left(\left[{ }^{(1)} \gamma\right]\right)[I]+{ }^{1} \kappa_{\underline{\underline{\Omega}}} \operatorname{tr}\left(\left[{ }^{(0)}{ }_{d} \bar{\sigma}\right]\right)[I] \\
& +\left(-\frac{{ }^{\sigma} b^{4}}{\sigma b^{2}}\right)\left[{ }^{(0)}{ }_{d} \bar{\sigma}\right]^{2}-\left(\alpha_{t m}\right)_{\underline{\Omega}}\left(\bar{\theta}-\bar{\theta}_{\underline{\Omega}}\right)[I]
\end{aligned}
$$

We note that each term in (7.31) has dimension of stress, thus the coefficient of $\left[{ }^{(0)}{ }_{d} \bar{\sigma}\right]^{2}$ must have dimension of (1/stress) which is same as (time/dimension of viscosity), i.e. $\lambda_{\underline{\Omega}} / \eta_{\underline{\underline{\Omega}}}$. We choose

$$
-\frac{\sigma b^{4}}{\sigma b^{2}}=\frac{\lambda_{\underline{\Omega}}}{\eta_{\underline{\Omega}}} \alpha
$$

$\alpha$ being a dimensionless parameter called mobility factor. Therefore

$$
\begin{aligned}
{\left[{ }^{(0)}{ }_{d} \bar{\sigma}\right] } & +\lambda_{\underline{\underline{\Omega}}}\left[{ }^{(1)}{ }_{d} \bar{\sigma}\right]=\left.\bar{\sigma}_{0}\right|_{\underline{\Omega}}[I]+2 \eta_{\underline{\underline{\Omega}}}\left[{ }^{(1)} \gamma\right] \\
& +\kappa_{\underline{\underline{\Omega}}} \operatorname{tr}\left(\left[{ }^{(1)} \gamma\right]\right)[I]+{ }^{1} \kappa_{\underline{\underline{\Omega}}} \operatorname{tr}\left(\left[{ }^{(0)}{ }_{d} \bar{\sigma}\right]\right)[I] \\
& +\frac{\lambda_{\underline{\underline{\Omega}}}}{\eta_{\underline{\underline{\Omega}}}} \alpha\left[{ }^{(0)}{ }_{d} \bar{\sigma}\right]^{2}-\left(\alpha_{t m}\right)_{\underline{\Omega}}\left(\bar{\theta}-\bar{\theta}_{\underline{\Omega}}\right)[I]
\end{aligned}
$$

(iv) In the derivation of the standard Giesekus model [2], we further assume that: (a) the initial stress field associated with the known configuration $\underline{\Omega}$, i.e. $\left.\bar{\sigma}_{0}\right|_{\Omega}[I]$, is zero (b) the stress field due to thermal expansion and contraction between the current and known configurations, i.e. the last term in (7.33), is neglected and (c) we further assume that ${ }^{1} \kappa_{\underline{\Omega}} \operatorname{tr}\left(\left[{ }^{(0)} \bar{\sigma}\right]\right)$ can be neglected. Thus we obtain

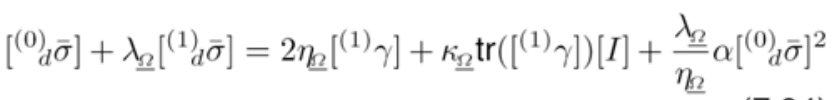

in which $\lambda_{\underline{2}}$ is called relaxation time, $\eta_{2}$ is first viscosity, $\kappa_{\Omega}$ is second viscosity and $\left(\alpha_{t m}\right)_{\Omega}$ is thermal modulus. (7.34) is the Giesekus constitutive model for compressible thermoviscoelastic fluids in which $\lambda_{\underline{2}}, \eta_{\underline{2}}, \kappa_{\underline{2}}$ and $\left(\alpha_{t m}\right)_{\Omega}$ are variable transport properties. If the fluid is incompressible, then the terms containing $\operatorname{tr}\left(\left[{ }^{(1)} \gamma\right]\right)$ are eliminated as for this case $\operatorname{tr}\left(\left[{ }^{(1)} \gamma\right]\right)=0$.

\subsubsection{Remarks}

1. The coefficients $\lambda_{\Omega}, \eta_{\Omega}, \kappa_{\Omega},\left(\alpha_{t m}\right)_{\Omega}$ are defined in the known configuration $\underline{\Omega}$ for which deformation is known, whereas all other quantities in (7.31) and (7.34) are in the current configuration. This is a consequence of Taylor series expansion of the coefficients about the known configuration $\underline{\Omega}$.

2. Based on (7.30), $\lambda_{\Omega}, \eta_{\Omega}, \kappa_{\Omega}$ etc. can be deformation dependent during evolution (keeping 1 . in mind) permitting experimental and/or empirical description of $\lambda_{\underline{2}}, \eta_{\underline{2}}, \kappa_{\underline{2}}$ etc. using their arguments given in (7.30). Thus, power law, Sutherland law etc. for $\lambda_{\underline{\Omega}}, \eta_{\underline{\Omega}}, \kappa_{\underline{\Omega}}$ etc. dependent on $\bar{\rho}_{\underline{\Omega}}$ and $\bar{\theta}_{\underline{\Omega}}$ are valid. Dependence of $\lambda_{\underline{\Omega}}, \eta_{\underline{\Omega}}, \kappa_{\underline{\underline{\Omega}}}$ etc. on $\left(\bar{I}^{j}\right)_{\underline{\Omega}} ; j=1,2, \ldots, 10$ allows us to represent variable shear thinning and shear thickening behaviors during the evolution. Power law, Carreau-Yasuda models etc. are valid based on (7.30) (contrary to the belief that these models do not have continuum mechanics foundation [2]).

3. By replacing $\left(\left[{ }^{(1)}{ }_{d} \bar{\sigma}\right],\left[{ }^{(0)}{ }_{d} \bar{\sigma}\right],\left[{ }^{(1)} \gamma\right]\right)$ with $\left(\left[{ }_{d} \bar{\sigma}^{(1)}\right]\right.$, $\left.\left[{ }_{d} \bar{\sigma}^{(0)}\right],\left[\gamma^{(1)}\right]\right),\left(\left[{ }_{d} \bar{\sigma}_{(1)}\right],\left[{ }_{d} \bar{\sigma}_{(0)}\right],\left[\gamma_{(1)}\right]\right)$ and $\left(\left[{ }^{(1)} \bar{\sigma}^{J}\right]\right.$, $\left.\left[{ }^{(0)} \bar{\sigma}^{J}\right],\left[{ }^{(1)} \gamma^{J}\right]\right)$ in (7.31) and (7.34), we obtain the Giesekus model that correspond to contravariant basis (upper convected), covariant basis (lower convected) and Jaumann rates. It is rather obvious that when the deformation is finite, all three rate constitutive equations will represent different physics, upper convected case being in most agreement with the physics of finite deformation [17].

4. If we assume that the known and current configurations are in close proximity of each other, then in (7.30) all coefficients can be expressed in terms of their arguments in the current configuration instead of in the known configuration $\underline{\Omega}$ (as shown in (7.30)). This is what is done in currently used models for variable material coefficients $[2,16]$. This is obviously not supported by the derivation presented here. By replacing the known configuration $\underline{\Omega}$ with the current configuration in (7.30), the material coefficients become functions of the unknown deformation field. 
5. The constitutive theories for the heat vector ${ }^{(0)} \overline{\boldsymbol{q}}$ have already been presented. Generally, Fourier heat conduction law (section 7.1) is commonly used in the majority of the published work.

6. It is important to note that the constitutive model (7.34) uses the first convected time derivative of the deviatoric Cauchy stress tensor as a dependent variable in the development of the constitutive theory, thus (7.34) are the constitutive equations in deviatoric Cauchy stress tensor $\left[{ }^{(0)} \bar{\sigma} \bar{\sigma}\right]$ and its convected time derivative. This derivation is supported by the axioms and principles of continuum mechanics and the constitutive theory. In the presently used Giesekus constitutive model, this is not the case. We present details in the following.

\subsubsection{Discussion on the Giesekus Constitutive Model Presented in this Paper and the Model Used Currently}

We note that the entropy inequality requires decomposition of the Cauchy stress tensor (in contra- or covariant or Jaumann basis) into equilibrium stress and deviatoric stress. The constitutive theory for the equilibrium stress using entropy inequality results in thermodynamic pressure $\bar{p}(\bar{\rho}, \bar{\theta})$ for compressible thermoviscoelastic fluids and mechanical pressure $\bar{p}(\bar{\theta})$ for incompressible case. Since the entropy inequality only requires the work expanded due to the deviatoric Cauchy stress to be positive but provides no mechanism for establishing the constitutive theory for it, the theory of invariants and generators is used for deriving the constitutive theory for it. The use of the deviatoric Cauchy stress tensor in the Giesekus constitutive model derived here is necessitated due to the entropy inequality. In the currently used Giesekus constitutive model for the stress tensor, the deviatoric Cauchy stress is further decomposed into solvent stress and polymer stress. If we consider contravariant basis, then

$$
\left[{ }_{d} \bar{\sigma}^{(0)}\right]=\left[{ }_{d} \bar{\sigma}^{(0)}\right]_{s}+\left[{ }_{d} \bar{\sigma}^{(0)}\right]_{p}
$$

in which $s$ and $p$ stand for solvent and polymer. The currently used Giesekus constitutive model has exactly the same form as the model presented here but uses $\left[{ }_{d} \bar{\sigma}^{(0)}\right]_{p}$ instead of $\left[{ }_{d} \bar{\sigma}^{(0)}\right]$ and is derived using Brownian motion of polymer molecules and kinetic theory [2,18]. For the solvent stress $\left[{ }_{d} \bar{\sigma}^{(0)}\right]_{s}$, Newton's law of viscosity is assumed as a constitutive theory. We note the following:

1. If we use the decomposition shown above and substitute it in the conditions resulting from the entropy inequality we still have the same restriction that the conversion of mechanical energy due to both solvent and polymer deviatoric Cauchy stress tensors be positive, but we have no mechanism for deriving constitutive theories for either one of them.
2. If we derive the Giesekus constitutive model using the theory of invariants and generators using $\left[{ }_{d} \bar{\sigma}^{(0)}\right]_{p}$ as a dependent variable in the constitutive theory and if we assume Newton's law of viscosity for $\left[{ }_{d} \bar{\sigma}^{(0)}\right]_{s}$, then of course we would obtain exactly the same Giesekus constitutive model as used currently. The question is "Is this permissible within the framework of the axioms of the constitutive theory and principles of continuum mechanics?".

3. Based on the axioms of the constitutive theory and the entropy inequality, $\left[{ }_{d} \bar{\sigma}^{(0)}\right]$ is a fundamental dependent variable in the rate constitutive theory for thermoviscoelastic fluids and hence must be used as dependent variable in the derivation of the rate theory.

4. If we follow 2, i.e. if we use $\left[{ }_{d} \bar{\sigma}^{(0)}\right]_{p}$ as a dependent variable in the rate theory for Giesekus constitutive model, then the constitutive theory for $\left[{ }_{d} \bar{\sigma}^{(0)}\right]_{s}$ must be derivable as well from the entropy inequality (and not assuming Newton's law of viscosity for it). This is obviously not possible.

5. Thus, based on the work presented here, we conclude that the use of the deviatoric Cauchy stress tensor (in a chosen basis) as a dependent variable is necessary in the derivation of the Giesekus constitutive model. This is consistent with the conditions resulting from the entropy inequality and the axioms of the constitutive theory based on continuum mechanics. Furthermore, there is no justification based on the entropy inequality for the decomposition (7.35) as $\left[{ }_{d} \bar{\sigma}^{(0)}\right]_{s}$ is not derivable using the conditions resulting from the entropy inequality. The use of Newton's law of viscosity may be a good engineering assumption but it has no basis in view of the entropy inequality and the axioms and principles of the constitutive theory in continuum mechanics.

6. It is rather obvious that the use of the Giesekus constitutive model presented in this paper and that used currently in the mathematical models derived using conservation laws of deforming thermoviscoelastic fluids will undoubtedly produce different behaviors.

\section{NUMERICAL STUDIES}

In this section we consider fully developed flow of a incompressible Giesekus fluid between parallel plates as model problem.

We use contravariant Cauchy stress tensor and Almansi strain tensors as conjugate measures of the stress and strain tensors in Eulerian description. If we decompose the contravariant Cauchy stress tensor in 
equilibrium stress and deviatoric contravariant Cauchy stress tensor, then the equilibrium stress is mechanical pressure $p$ and the deviatoric contravariant Cauchy stress tensor becomes a dependent variable in the constitutive theory. This yields upper convected Giesekus (UCG1) constitutive model. Numerical results are presented using the upper convected Giesekus constitutive model derived in this paper (UCG1) as well as using the currently used constitutive model in deviatoric polymer stress (UCG2).

Since the description is understood to be Eulerian, we drop over bar $\left(^{-}\right)$on all quantities for simplicity of notation and replace it with hat $\left(^{\wedge}\right)$ to emphasize that these quantities have dimensions. Quantities without hat $\left({ }^{\wedge}\right)$ are dimensionless. To conform to commonly used engineering notations we replace $x_{i} ; i=1,2,3$ by $x, y, z$ and $\bar{v}_{i} ; i=1,2,3$ by $u, v, w$ and ${ }^{(0)}{ }_{d} \bar{\sigma}_{i j} ; i, j=1,2,3$ by $\tau_{i j}$; $i, j=1,2,3$ in the mathematical model.

We consider an incompressible Giesekus fluid $\mathrm{PIB} / \mathrm{C} 14$ [19] with the following material coefficients (assumed constant).

$$
\begin{aligned}
& \hat{\rho}=800 \mathrm{~kg} / \mathrm{m}^{3} \quad ; \quad \hat{\eta}_{s}=0.002 \mathrm{~Pa} \mathrm{~s} \quad ; \quad \hat{\eta}_{p}=1.424 \mathrm{~Pa} \mathrm{~s} \\
& \hat{\eta}=1.426 \text { Pa s } \quad ; \quad \hat{\lambda}=0.06 \mathrm{~s} \quad ; \quad \alpha=0.15
\end{aligned}
$$

in which $\hat{\rho}, \hat{\eta}_{s}, \hat{\eta}_{p}, \hat{\eta}, \hat{\lambda}$ and $\alpha$ are density, solvent viscosity, polymer viscosity, total viscosity, relaxation time and mobility factor. For a fixed configuration and a given fluid we can study the influence of the constitutive models on the flow physics in at least two ways:

(i) For a fixed flow rate, the differences in the constitutive equation in the two models will produce different $\partial p / \partial x$ and other dependent variables in the two cases. As the flow rate increases, the differences in $\partial p / \partial x$ and the other dependent variables in the two cases are expected to increase as well.

(ii) In the second approach, we could choose a value of $\partial p / \partial x$ that is the same in both cases and compute results. Both models are bound to produce different velocity fields and hence different flow rates. For very low values of $\partial p / \partial x$ we expect the velocity field in the two cases to be not drastically different from each other but as $\partial p / \partial x$ increases, the differences are expected to be significant.

Obviously, (ii) is easier as it merely requires specification of $\partial p / \partial x$ as input and the rest of the detail of the flow are computed. We use this approach to study the influence of the two constitutive models (UCG1 and UCG2) on the flow physics of fully developed flow between parallel plates (model problem 1) and fully developed flow between parallel plates using a two dimensional formulation (model problem 2). It is obvious that both model problems will be in agreement when the same constitutive model is used.

\subsection{Model Problem 1: Fully Developed Flow Be- tween Parallel Plates}

Figure 1 shows a schematic using dimensionless quantities. The plates are separated by a distance $2 H$. The origin of the $x y$-coordinate is located at the center of the plates and the positive $x$-direction is the direction of the flow. The flow is pressure driven, i.e. $\partial p / \partial x$ (negative) is specified. The mathematical model describing the flow physics (for incompressible case with isothermal flow assumption) consists of $x$ - and $y$-momentum equations and the constitutive equations. The continuity equation in this case is satisfied identically.

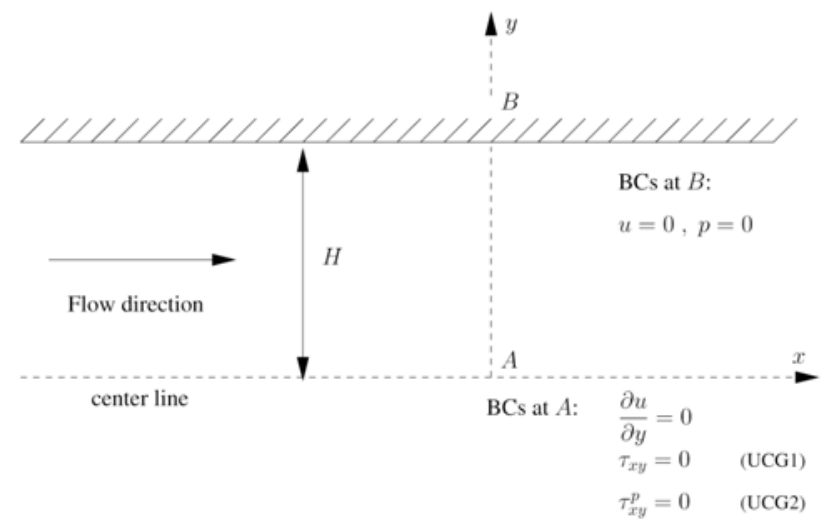

Figure 1: Schematic of 1-D fully developed flow between parallel plates (half domain)

We begin with all quantities with their usual dimensions (units) in the development of the mathematical model and then non-dimensionalize them using the following. The quantities with the subscript zero are the reference quantities.

$$
\begin{aligned}
& x=\frac{\hat{x}}{L_{0}}, y=\frac{\hat{y}}{L_{0}}, \eta=\frac{\hat{\eta}}{\eta_{0}}, \eta_{s}=\frac{\hat{\eta}_{s}}{\eta_{0}}, \eta_{p}=\frac{\hat{\eta}_{p}}{\eta_{0}} \\
& \rho=\frac{\hat{\rho}}{\rho_{0}}, u=\frac{\hat{u}}{u_{0}}, v=\frac{\hat{v}}{u_{0}}, p=\frac{\hat{p}}{p_{0}}, \boldsymbol{\tau}=\frac{\hat{\boldsymbol{\tau}}}{\tau_{0}} \\
& p_{0}=\tau_{0}= \begin{cases}\rho_{0} u_{0}^{2} & ; \text { Ch. kinetic energy } \\
\frac{\mu_{0} u_{0}}{L_{0}} & ; \text { or } \text { Ch. viscous stress }\end{cases}
\end{aligned}
$$

in which $\hat{u}, \hat{v}$ are velocities in the $x$ - and $y$-direction, $\hat{p}$ is mechanical pressure and $\hat{\boldsymbol{\tau}}$ is deviatoric stress tensor, all in the current configuration.1 We choose the larger of the two for $p_{0}$ (and $\tau_{0}$ ). This results in dimensionless form of the mathematical model given in the following: 


\section{Momentum Equations:}

In the absence of body forces

$$
\begin{aligned}
& \left(\frac{p_{0}}{\rho_{0} u_{0}^{2}}\right) \frac{\partial p}{\partial x}-\left(\frac{\tau_{0}}{\rho_{0} u_{0}^{2}}\right) \frac{\partial \tau_{x y}}{\partial y}=0 \\
& \left(\frac{p_{0}}{\rho_{0} u_{0}^{2}}\right) \frac{\partial p}{\partial y}-\left(\frac{\tau_{0}}{\rho_{0} u_{0}^{2}}\right) \frac{\partial \tau_{y y}}{\partial y}=0
\end{aligned}
$$

\section{Giesekus Constitutive Model:}

We consider the upper convected Giesekus constitutive model derived in this paper (UCG1) and the upper convected Giesekus constitutive model used currently (UCG2).

\section{UCG1:}

In this model, the first convected time derivative of $\boldsymbol{\tau}$, the deviatoric contravariant Cauchy stress tensor, is a dependent variable in the constitutive theory. Dimensionless form of the constitutive model is given by

$$
\begin{gathered}
\tau_{x x}-2 D e \tau_{x y} \frac{\partial u}{\partial y}-\alpha \frac{D e}{\eta}\left(\frac{L_{0} \tau_{0}}{u_{0} \eta_{0}}\right)\left(\left(\tau_{x x}\right)^{2}+\left(\tau_{x y}\right)^{2}\right)=0 \\
\tau_{y y}-\alpha \frac{D e}{\eta}\left(\frac{L_{0} \tau_{0}}{u_{0} \eta_{0}}\right)\left(\left(\tau_{y y}\right)^{2}+\left(\tau_{x y}\right)^{2}\right)=0 \\
\tau_{x y}-D e \tau_{y y} \frac{\partial u}{\partial y}-\alpha \frac{D e}{\eta}\left(\frac{L_{0} \tau_{0}}{u_{0} \eta_{0}}\right) \tau_{x y}\left(\tau_{x x}+\tau_{y y}\right)= \\
\eta\left(\frac{u_{0} \eta_{0}}{L_{0} \tau_{0}}\right) \frac{\partial u}{\partial y}
\end{gathered}
$$

Equations (8.2) - (8.4) constitute the complete mathematical model in dependent variables $u, p, \tau_{x x}, \tau_{y y}$ and $\tau_{x y}$ for fully developed flow between parallel plates when using UCG1.

\section{UCG2:}

This constitutive model is used currently [2]. In this model $\boldsymbol{\tau}$ is decomposed into solvent and polymer stresses.

$$
\boldsymbol{\tau}=\boldsymbol{\tau}^{s}+\boldsymbol{\tau}^{p}
$$

The Newton's law of viscosity is assumed as a constitutive model for $\boldsymbol{\tau}^{s} . \tau_{x x}^{s}$ and $\tau_{y y}^{s}$ are zero for this model problem and we only have $\tau_{x y}^{s}$ in the constitute model for solvent stress.

$$
\tau_{x y}^{s}=\left(\frac{u_{0} \eta_{0}}{L_{0} \tau_{0}}\right) \eta_{s} \frac{\partial u}{\partial y}
$$

and hence, from (8.5)

$$
\begin{aligned}
\tau_{x x} & =\tau_{x x}^{p} \quad ; \quad \tau_{y y}=\tau_{y y}^{p} \\
\tau_{x y} & =\tau_{x y}^{p}+\left(\frac{u_{0} \eta_{0}}{L_{0} \tau_{0}}\right) \eta_{s} \frac{\partial u}{\partial y}
\end{aligned}
$$

For polymer stress $\boldsymbol{\tau}^{p}$, the dimensionless form of the constitutive equations are given by (obtained by replacing $\boldsymbol{\tau}$ with $\boldsymbol{\tau}^{p}$ and $\eta$ by $\eta_{p}$ in (8.4)) the following [2]:

$$
\begin{gathered}
\tau_{x x}^{p}-2 D e \tau_{x y}^{p} \frac{\partial u}{\partial y}-\alpha \frac{D e}{\eta_{p}}\left(\frac{L_{0} \tau_{0}}{u_{0} \eta_{0}}\right)\left(\left(\tau_{x x}^{p}\right)^{2}+\left(\tau_{x y}^{p}\right)^{2}\right)=0 \\
\tau_{y y}^{p}-\alpha \frac{D e}{\eta_{p}}\left(\frac{L_{0} \tau_{0}}{u_{0} \eta_{0}}\right)\left(\left(\tau_{y y}^{p}\right)^{2}+\left(\tau_{x y}^{p}\right)^{2}\right)=0 \\
\tau_{x y}^{p}-D e \tau_{y y}^{p} \frac{\partial u}{\partial y}-\alpha \frac{D e}{\eta_{p}}\left(\frac{L_{0} \tau_{0}}{u_{0} \eta_{0}}\right) \tau_{x y}^{p}\left(\tau_{x x}^{p}+\tau_{y y}^{p}\right)= \\
\eta_{p}\left(\frac{u_{0} \eta_{0}}{L_{0} \tau_{0}}\right) \frac{\partial u}{\partial y}
\end{gathered}
$$

Using (8.7) in the momentum equations (8.2) and (8.3), we can express the momentum equations in terms of $\tau_{y y}^{p}, \tau_{x y}^{p}$ and velocity gradients

$$
\begin{aligned}
& \left(\frac{p_{0}}{\rho_{0} u_{0}^{2}}\right) \frac{\partial p}{\partial x}-\left(\frac{\tau_{0}}{\rho_{0} u_{0}^{2}}\right) \frac{\partial \tau_{x y}^{p}}{\partial y}-\left(\frac{\eta_{0}}{L_{0} \rho_{0} u_{0}}\right) \eta_{s} \frac{\partial^{2} u}{\partial y^{2}}=0 \\
& \left(\frac{p_{0}}{\rho_{0} u_{0}^{2}}\right) \frac{\partial p}{\partial y}-\left(\frac{\tau_{0}}{\rho_{0} u_{0}^{2}}\right) \frac{\partial \tau_{y y}^{p}}{\partial y}=0
\end{aligned}
$$

(8.8) - (8.10) constitute the complete mathematical model in dependent variables $u, p, \tau_{x x}^{p}, \tau_{y y}^{p}$ and $\tau_{x y}^{p}$ for fully developed flow between parallel plates when using UCG2.

\section{Solutions of the BVPS:}

In this section we consider solutions of the BVPs described by (8.2) - (8.4) for UCG1 and (8.8) - (8.10) for UCG2. Since $\partial p / \partial x$ is constant (specified), from (8.2) we can determine $\tau_{x y}$ by integrating with respect to $y$ and using the boundary condition $\tau_{x y}=0$ at $y=0$ (due to symmetry)

$$
\tau_{x y}=\left(\frac{\partial p}{\partial x}\right) y
$$

A theoretical solution for the remaining dependent variables is not readily possible due to the complexity of the constitutive equations in both boundary value problems (UCG1 and UCG2), hence we consider their numerical solutions using finite element processes based on the residual functional (least squares finite element method). The local approximations are considered in higher order spaces $H^{k, p}\left(\bar{\Omega}_{x}^{e}\right)$ in which $\bar{\Omega}_{x}^{e}$ is the spatial domain of a typical element ' $e$ ' of the discretization. The resulting non-linear algebraic equations from the least squares process are solved using Newton's linear method. The computational processes in this approach are unconditionally stable and permit higher order global differentiability local approximations. See reference [20-22] for details of local approximations and the least squares process for non-linear PDEs and higher order spaces. In the computations of the numerical solutions we choose

$$
\begin{aligned}
& \hat{H}=L_{0}=3.175 \mathrm{~mm} \quad, \quad \rho_{0}=\hat{\rho}=800 \mathrm{~kg} / \mathrm{m}^{3} \\
& \eta_{0}=\hat{\eta}=1.426 \mathrm{~Pa} \mathrm{~s} \quad, \quad u_{0}=0.5 \mathrm{~m} / \mathrm{s}
\end{aligned}
$$


which gives

$$
\begin{aligned}
& H=1 ; \quad p_{0}=\tau_{0}=\rho_{0} u_{0}^{2}=200 \mathrm{~Pa} \\
& R e=\frac{\rho_{0} L_{0} u_{0}}{\eta_{0}}=0.8906, D e=\frac{\hat{\lambda} u_{0}}{L_{0}}=9.45
\end{aligned}
$$

or $D e=\hat{\lambda} u_{\max } / L_{0}=18.89764 u_{\max }$. A good discretization of the spatial domain $0 \leq y \leq 1$ is important in ensuring satisfactory convergence of the Newton's linear method for the system of non-linear algebraic equations and good accuracy of computed solutions. With progressively increasing $\partial p / \partial x$, we expect development of a constant velocity core at the center of the flow. This suggests the use of a highly biased finer discretization towards the walls. A two element graded mesh with element length of 0.2 and 0.8 starting from the wall (see Figure 2) is used in and local approximations are $p$-version (3-node elements) in higher order spaces.

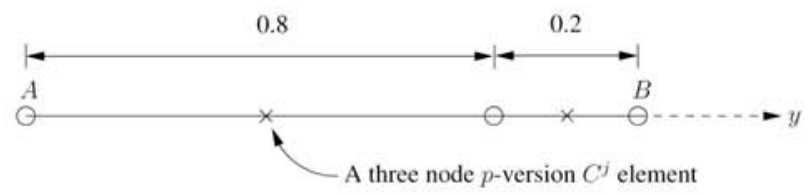

Figure 2: Graded mesh discretization using two 3-node $p$ version elements

Initial $p$-convergence studies with this discretization suggest $p=9$ with $k=2$, local approximations of class $C^{1}\left(\bar{\Omega}_{x}^{e}\right)$, to be sufficient for good accuracy of results. For this choice of mesh, $p$-level $(p=9)$ and order of the space $(k=2)$, the residual or least squares functional values remain $O\left(10^{-8}\right)-O\left(10^{-20}\right)$ indicating that the PDEs are satisfied very accurately (in the pointwise sense for UCG1 as the integrals are Riemann, and not strictly in the pointwise sense for UCG2 since the integrals are Lebesgue) when local approximations for $u, p, \tau_{x x}, \tau_{y y}$ and $\tau_{x y}$ are of class $C^{1}\left(\bar{\Omega}_{x}^{e}\right)$. Newton's linear method used for solving the non-linear algebraic equations converges in less than 10 iterations for all numerical studies presented here. In the numerical studies we begin with $\partial p / \partial x=-0.1$ for which a converged solution is obtained and then progressively increase it up to $\partial p / \partial x=-0.275$ using a continuation procedure in which converged solutions at lower $\partial p / \partial x$ are used as initial (or starting) solution in the Newton's linear method.

Figure 3 shows graphs of velocity $u$ versus $y$ for different values of $\partial p / \partial x$ for both UCG1 and UCG2. Graphs of velocity gradient $\partial u / \partial y$ versus $y$ for different values of $\partial p / \partial x$ are shown in Figure 4. For $\partial p / \partial x$ values up to -0.2 , the results from both UCG1 and UCG2 are in good agreement (Figures $\mathbf{3}$ and $\mathbf{4}$ ). Beyond $\partial p / \partial x$ values of -0.2 , the results from the two BVPs begin to deviate. Higher values of $\partial p / \partial x$ result in larger deviations between the two models. At $\partial p / \partial x=-0.275, u_{\max }$ at $y=0$ from UCG1 is more than twice of $u_{\max }$ at $y=0$ from UCG2. This of course implies drastically different flow rates resulting from the two models for the same pressure gradient.

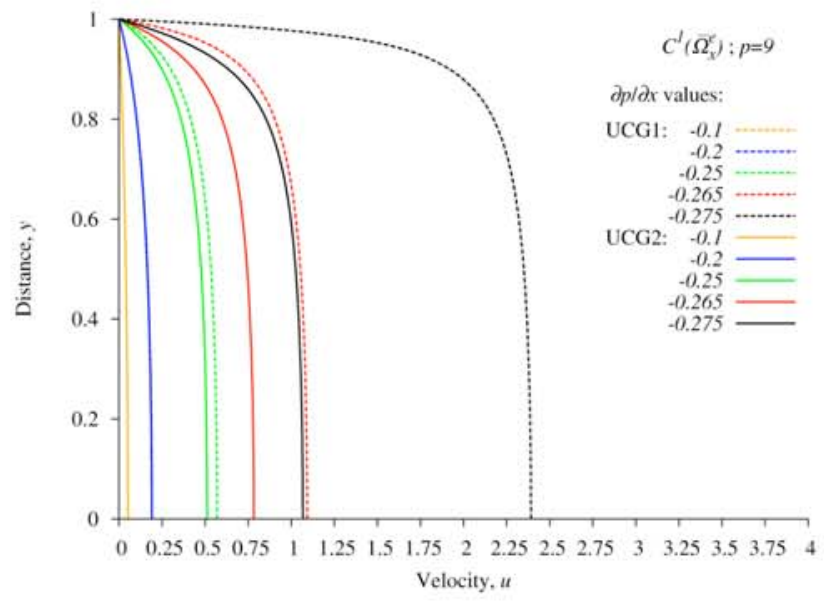

Figure 3: Velocity $u$ versus distance $y$

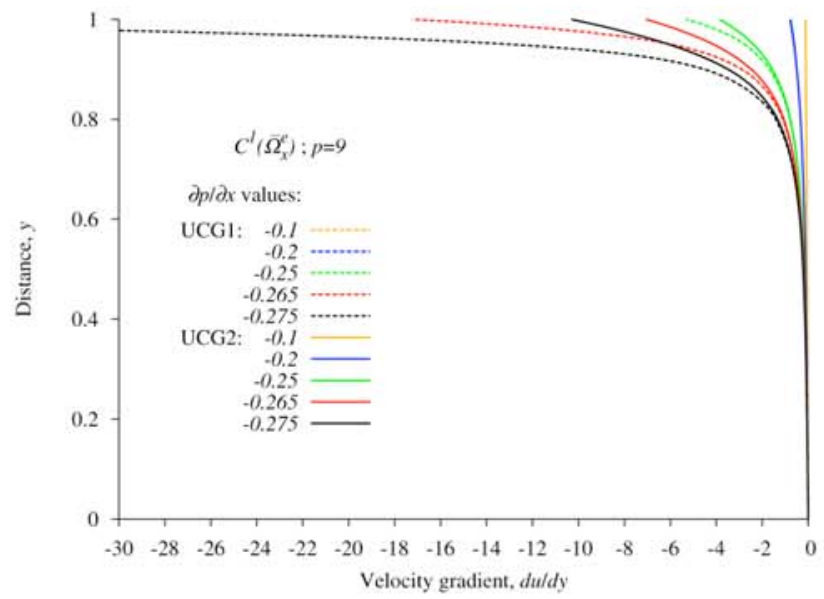

Figure 4: Velocity gradient $d u / d y$ versus distance $y$

Figures 5 - 7 show plots of $\tau_{x x}, \tau_{y y}$ and $\tau_{x y}$ versus $y$ for both UCG1 and UCG2. For $\partial p / \partial x$ values beyond -0.2 we observe progressively increasing deviations between solutions obtained from the two BVPs for $\tau_{x x}$ and $\tau_{y y}$. For $\partial p / \partial x=-0.275, \tau_{x x}$ and $\tau_{y y}$ from UCG1 are roughly more than twice of those from UCG2. Computed $\tau_{x y}$ from the numerical solutions of both BVPs are in perfect agreement with the theoretical solution (8.11) for all values of $\partial p / \partial x$ as $\tau_{x y}$ only depends upon $\partial p / \partial x$ which is same in both models. The residual $(I)$ values of $O\left(10^{-8}\right)$ or lower and the use of $C^{1}\left(\bar{\Omega}_{x}^{e}\right)$ ensure that the computed solutions satisfy the GDEs accurately. 


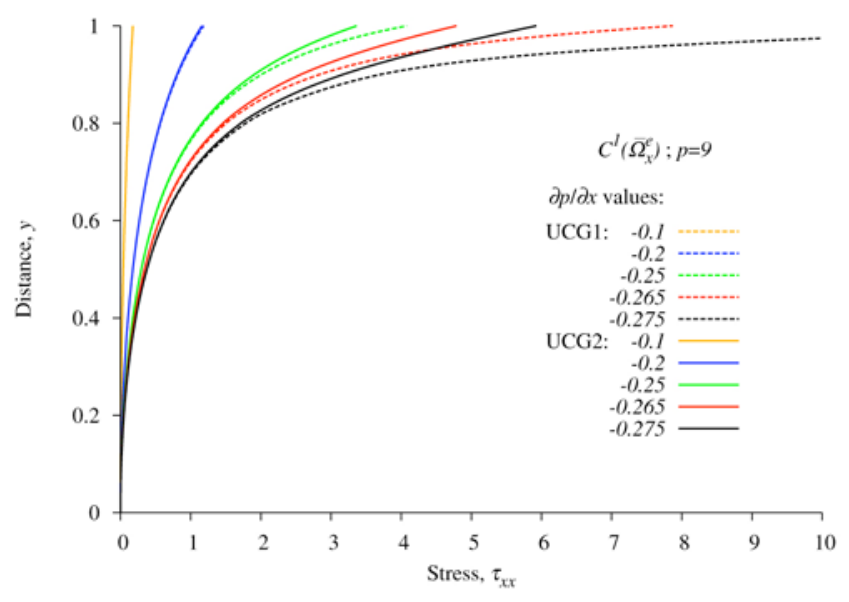

Figure 5: Stress component $\tau_{x x}$ versus distance $y$

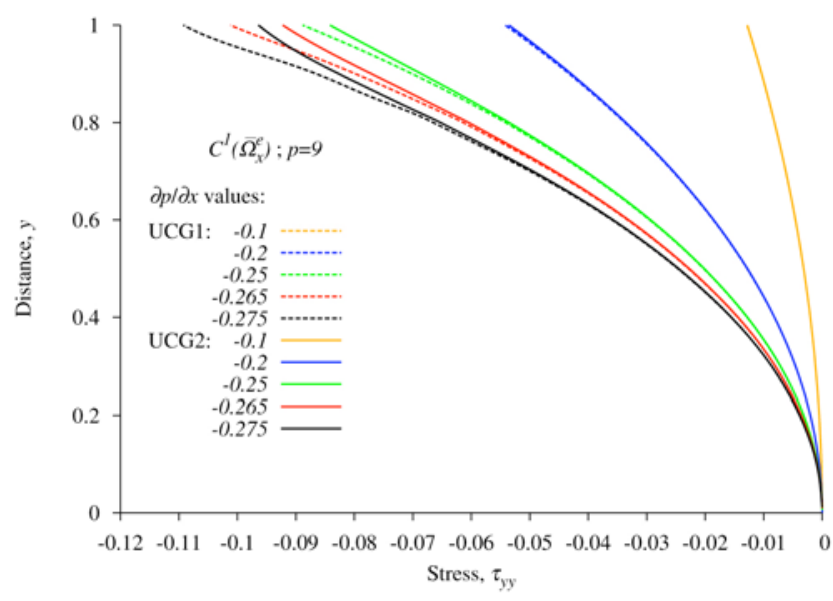

Figure 6: Stress component $\tau_{y y}$ versus distance $y$

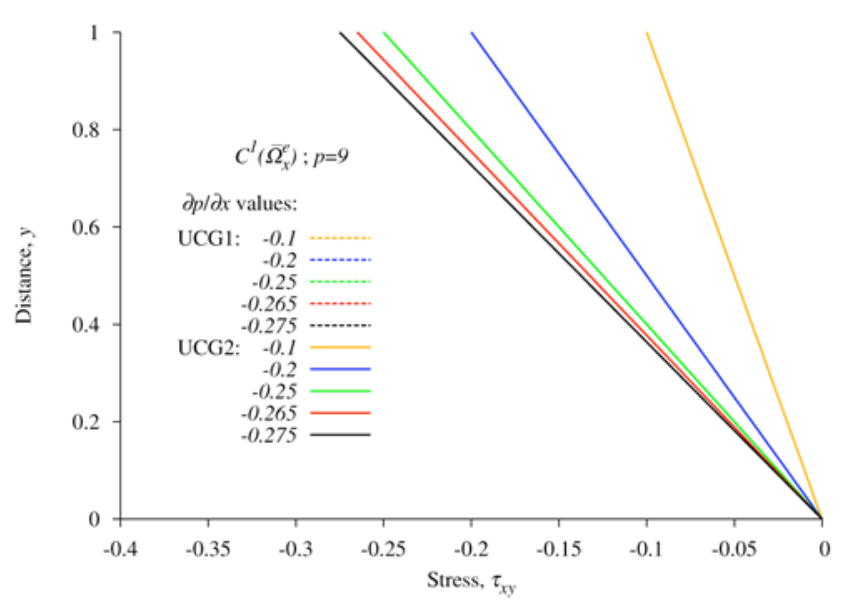

Figure 7: Stress component $\tau_{x y}$ versus distance $y$

\subsection{Model Problem 2: Fully Developed Flow Be- tween Parallel Plates using 2D Formulation}

In this numerical study we consider the same model problem as considered for model problem 1, i.e. fully developed flow between parallel plates but we use 2D mathematical model. The purpose of this study is to show performance of full mathematical model and to demonstrate that for fully developed flow between parallel plates, this full model produces precisely same results as degenerate model used in section 8.1. Figure 8 shows a schematic using dimensionless quantities in which $A B C D$ is the computational domain. Origin of the coordinate system $x, y$ is located at $A$. Positive $x$ direction is the direction of the flow.

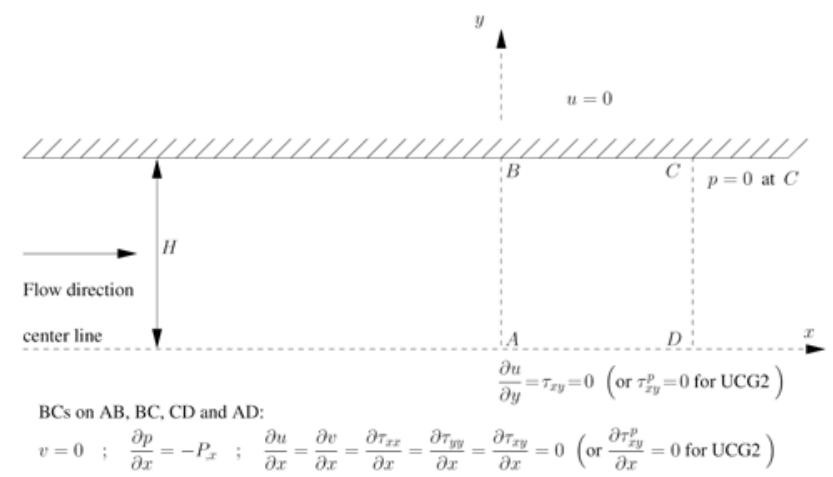

Figure 8: Schematic of 2-D fully developed flow between parallel plates (half domain)

In this case, the mathematical model describing the flow physics (for incompressible case with isothermal flow assumption) consists of continuity equation, $x$ - and $y$-momentum equations and the constitutive equations. We begin with all quantities with their usual dimensions (units) in the development of the mathematical model and then non-dimensionalize them using (8.1) in which $\hat{u}, \hat{v}$ are velocities in the $x$ - and $y$-direction, $\hat{p}$ is mechanical pressure and $\hat{\boldsymbol{\tau}}$ is deviatoric stress tensor, all in the current configuration. We choose the larger of the two for $p_{0}$ (and $\tau_{0}$ ). This results in the following dimensionless form of the mathematical model:

\section{Continuity Equation:}

$$
\rho\left(\frac{\partial u}{\partial x}+\frac{\partial v}{\partial y}\right)=0
$$

\section{Momentum Equations:}

In the absence of body forces 


$$
\begin{aligned}
\rho\left(u \frac{\partial u}{\partial x}+v \frac{\partial u}{\partial y}\right)+\left(\frac{p_{0}}{\rho_{0} u_{0}^{2}}\right) \frac{\partial p}{\partial x} \\
-\left(\frac{\tau_{0}}{\rho_{0} u_{0}^{2}}\right)\left(\frac{\partial \tau_{x x}}{\partial x}+\frac{\partial \tau_{x y}}{\partial y}\right)=0 \\
\rho\left(u \frac{\partial v}{\partial x}+v \frac{\partial v}{\partial y}\right)+\left(\frac{p_{0}}{\rho_{0} u_{0}^{2}}\right) \frac{\partial p}{\partial y} \\
-\left(\frac{\tau_{0}}{\rho_{0} u_{0}^{2}}\right)\left(\frac{\partial \tau_{x y}}{\partial x}+\frac{\partial \tau_{y y}}{\partial y}\right)=0
\end{aligned}
$$

\section{Giesekus Constitutive Model:}

We consider the upper convected Giesekus constitutive model derived in this paper (UCG1) and the upper convected Giesekus constitutive model used currently (UCG2).

\section{UCG1:}

In this model, the first convected time derivative of $\boldsymbol{\tau}$, the deviatoric contravariant Cauchy stress tensor, is a dependent variable in the constitutive theory. Dimensionless form of the constitutive model is given by

$$
\begin{aligned}
& \tau_{x x}+D e\left(u \frac{\partial \tau_{x x}}{\partial x}+v \frac{\partial \tau_{x x}}{\partial y}-2 \tau_{x y} \frac{\partial u}{\partial y}-2 \tau_{x x} \frac{\partial u}{\partial x}\right) \\
& -\alpha \frac{D e}{\eta}\left(\frac{L_{0} \tau_{0}}{u_{0} \eta_{0}}\right)\left(\left(\tau_{x x}\right)^{2}+\left(\tau_{x y}\right)^{2}\right)=2 \eta\left(\frac{u_{0} \eta_{0}}{L_{0} \tau_{0}}\right) \frac{\partial u}{\partial x} \\
& \tau_{y y}+D e\left(u \frac{\partial \tau_{y y}}{\partial x}+v \frac{\partial \tau_{y y}}{\partial y}-2 \tau_{x y} \frac{\partial v}{\partial x}-2 \tau_{y y} \frac{\partial v}{\partial y}\right) \\
& -\alpha \frac{D e}{\eta}\left(\frac{L_{0} \tau_{0}}{u_{0} \eta_{0}}\right)\left(\left(\tau_{y y}\right)^{2}+\left(\tau_{x y}\right)^{2}\right)=2 \eta\left(\frac{u_{0} \eta_{0}}{L_{0} \tau_{0}}\right) \frac{\partial v}{\partial y} \\
& \tau_{x y}+D e\left(u \frac{\partial \tau_{x y}}{\partial x}+v \frac{\partial \tau_{x y}}{\partial y}-\tau_{x y}\left(\frac{\partial u}{\partial x}+\frac{\partial v}{\partial y}\right)\right. \\
& \left.-\tau_{x x} \frac{\partial v}{\partial x}-\tau_{y y} \frac{\partial u}{\partial y}\right)-\alpha \frac{D e}{\eta}\left(\frac{L_{0} \tau_{0}}{u_{0} \eta_{0}}\right) \tau_{x y}\left(\tau_{x x}+\tau_{y y}\right) \\
& =\eta\left(\frac{u_{0} \eta_{0}}{L_{0} \tau_{0}}\right)\left(\frac{\partial u}{\partial y}+\frac{\partial v}{\partial x}\right)
\end{aligned}
$$

Equations (8.12) - (8.14) constitute the complete mathematical model in dependent variables $u, v, p, \tau_{x x}, \tau_{y y}$ and $\tau_{x y}$ for two dimensional steady flow using the constitutive model UCG1.

\section{UCG2:}

This constitutive model is used currently [2]. As in model problem 1, here also, $\boldsymbol{\tau}$ is decomposed into solvent and polymer stresses.

$$
\boldsymbol{\tau}=\boldsymbol{\tau}^{s}+\boldsymbol{\tau}^{p}
$$

and the Newton's law of viscosity is assumed as a constitutive theory for $\boldsymbol{\tau}^{s}$.

$$
\begin{aligned}
\tau_{x x}^{s} & =2\left(\frac{u_{0} \eta_{0}}{L_{0} \tau_{0}}\right) \eta_{s} \frac{\partial u}{\partial x} \\
\tau_{y y}^{s} & =2\left(\frac{u_{0} \eta_{0}}{L_{0} \tau_{0}}\right) \eta_{s} \frac{\partial v}{\partial y} \\
\tau_{x y}^{s} & =\left(\frac{u_{0} \eta_{0}}{L_{0} \tau_{0}}\right) \eta_{s}\left(\frac{\partial u}{\partial y}+\frac{\partial v}{\partial x}\right)
\end{aligned}
$$

and hence, from (8.15)

$$
\begin{aligned}
& \tau_{x x}=\tau_{x x}^{p}+2\left(\frac{u_{0} \eta_{0}}{L_{0} \tau_{0}}\right) \eta_{s} \frac{\partial u}{\partial x} \\
& \tau_{y y}=\tau_{y y}^{p}+2\left(\frac{u_{0} \eta_{0}}{L_{0} \tau_{0}}\right) \eta_{s} \frac{\partial v}{\partial y} \\
& \tau_{x y}=\tau_{x y}^{p}+\left(\frac{u_{0} \eta_{0}}{L_{0} \tau_{0}}\right) \eta_{s}\left(\frac{\partial u}{\partial y}+\frac{\partial v}{\partial x}\right)
\end{aligned}
$$

For polymer stress $\boldsymbol{\tau}^{p}$, the dimensionless form of the constitutive equations are given by (obtained by replacing $\boldsymbol{\tau}$ with $\boldsymbol{\tau}^{p}$ and $\eta$ by $\eta_{p}$ in (8.14)) the following [2]:

$$
\begin{aligned}
\tau_{x x}^{p}+ & D e\left(u \frac{\partial \tau_{x x}^{p}}{\partial x}+v \frac{\partial \tau_{x x}^{p}}{\partial y}-2 \tau_{x y}^{p} \frac{\partial u}{\partial y}-2 \tau_{x x}^{p} \frac{\partial u}{\partial x}\right) \\
& -\alpha \frac{D e}{\eta_{p}}\left(\frac{L_{0} \tau_{0}}{u_{0} \eta_{0}}\right)\left(\left(\tau_{x x}^{p}\right)^{2}+\left(\tau_{x y}^{p}\right)^{2}\right)=2 \eta_{p}\left(\frac{u_{0} \eta_{0}}{L_{0} \tau_{0}}\right) \frac{\partial u}{\partial x} \\
\tau_{y y}^{p}+ & D e\left(u \frac{\partial \tau_{y y}^{p}}{\partial x}+v \frac{\partial \tau_{y y}^{p}}{\partial y}-2 \tau_{x y}^{p} \frac{\partial v}{\partial x}-2 \tau_{y y}^{p} \frac{\partial v}{\partial y}\right) \\
& -\alpha \frac{D e}{\eta_{p}}\left(\frac{L_{0} \tau_{0}}{u_{0} \eta_{0}}\right)\left(\left(\tau_{y y}^{p}\right)^{2}+\left(\tau_{x y}^{p}\right)^{2}\right)=2 \eta_{p}\left(\frac{u_{0} \eta_{0}}{L_{0} \tau_{0}}\right) \frac{\partial v}{\partial y} \\
\tau_{x y}^{p}+ & D e\left(u \frac{\partial \tau_{x y}^{p}}{\partial x}+v \frac{\partial \tau_{x y}^{p}}{\partial y}-\tau_{x y}^{p}\left(\frac{\partial u}{\partial x}+\frac{\partial v}{\partial y}\right)-\tau_{x x}^{p} \frac{\partial v}{\partial x}\right. \\
& \left.-\tau_{y y}^{p} \frac{\partial u}{\partial y}\right)-\alpha \frac{D e}{\eta_{p}}\left(\frac{L_{0} \tau_{0}}{u_{0} \eta_{0}}\right) \tau_{x y}^{p}\left(\tau_{x x}^{p}+\tau_{y y}^{p}\right) \\
= & \eta_{p}\left(\frac{u_{0} \eta_{0}}{L_{0} \tau_{0}}\right)\left(\frac{\partial u}{\partial y}+\frac{\partial v}{\partial x}\right)
\end{aligned}
$$

Using (8.17) in the momentum equations (8.13), we can express the momentum equations in terms of $\tau_{x x}^{p}$, $\tau_{y y}^{p}, \tau_{x y}^{p}$ and velocity gradients

$$
\begin{gathered}
\rho\left(u \frac{\partial u}{\partial x}+v \frac{\partial u}{\partial y}\right)+\left(\frac{p_{0}}{\rho_{0} u_{0}^{2}}\right) \frac{\partial p}{\partial x}-\left(\frac{\tau_{0}}{\rho_{0} u_{0}^{2}}\right)\left(\frac{\partial \tau_{x x}^{p}}{\partial x}+\frac{\partial \tau_{x y}^{p}}{\partial y}\right) \\
-\left(\frac{\eta_{0}}{L_{0} \rho_{0} u_{0}}\right) \eta_{s}\left(2 \frac{\partial^{2} u}{\partial x^{2}}+\frac{\partial^{2} u}{\partial y^{2}}+\frac{\partial^{2} v}{\partial y \partial x}\right)=0 \\
\rho\left(u \frac{\partial v}{\partial x}+v \frac{\partial v}{\partial y}\right)+\left(\frac{p_{0}}{\rho_{0} u_{0}^{2}}\right) \frac{\partial p}{\partial y}-\left(\frac{\tau_{0}}{\rho_{0} u_{0}^{2}}\right)\left(\frac{\partial \tau_{x y}^{p}}{\partial x}+\frac{\partial \tau_{y y}^{p}}{\partial y}\right) \\
-\left(\frac{\eta_{0}}{L_{0} \rho_{0} u_{0}}\right) \eta_{s}\left(2 \frac{\partial^{2} v}{\partial y^{2}}+\frac{\partial^{2} v}{\partial x^{2}}+\frac{\partial^{2} u}{\partial y \partial x}\right)=0
\end{gathered}
$$

Equations (8.12), (8.18), (8.19) constitute the complete mathematical model in dependent variables $u, v$, 
$p, \tau_{x x}^{p}, \tau_{y y}^{p}$ and $\tau_{x y}^{p}$ for two dimensional steady flow using the constitutive model UCG2, used currently for incompressible Giesekus fluids.

\section{Solutions of the BVPs:}

In this section we consider solutions of the BVPs described by(8.12) - (8.14) for UCG1 and (8.12), (8.18), (8.19) for UCG2. A theoretical solution for dependent variables is not possible due to the complexity of the constitutive equations in both boundary value problems, hence we consider their numerical solutions using finite element processes based on the residual functional (least squares finite element method) in which the resulting non-linear algebraic equations from the least squares process are solved using Newton's linear method. The computational processes in this approach are unconditionally stable and permit higher order global differentiability local approximations. Details of the local approximations and the least squares finite element processes for non-linear PDEs and higher order spaces can be found in references [20-25]. The local approximations are considered in higher order spaces $H^{k, p}\left(\bar{\Omega}_{x y}^{e}\right)$ in which $\bar{\Omega}_{x y}^{e}$ is the spatial domain of a typical element ' $e$ ' of the discretization. In the computations of the numerical solutions we choose

$$
\begin{aligned}
& \hat{H}=L_{0}=3.175 \mathrm{~mm} \quad, \quad \rho_{0}=\hat{\rho}=800 \mathrm{~kg} / \mathrm{m}^{3} \\
& \eta_{0}=\hat{\eta}=1.426 \mathrm{~Pa} \mathrm{~s} \quad, \quad u_{0}=0.5 \mathrm{~m} / \mathrm{s}
\end{aligned}
$$

where $H=1, P_{0}=200 \mathrm{~Pa}, R e=0.8906$ and $D e=9.45$, same as in model problem 1 .

In this case, the rectangular domain $A B C D$ is discretized using two 9-node $p$-version elements of lengths 0.2 and 0.8 (Figure 9) in the $y$-direction. Length $A D$ is chosen as 1.0 (arbitrary). The local approximations are considered to be of equal degree for all variables. We consider $p=\left(p_{1}, p_{2}\right)=(9,9)$ with $k=\left(k_{1}, k_{2}\right)=(2,2)$, i.e. local approximations of class $C^{1,1}\left(\bar{\Omega}_{x y}^{e}\right)$. For this choice of mesh, $p$-level and order of space, the residual functional values are of orders of $O\left(10^{-8}\right)-O\left(10^{-16}\right)$ indicating that the PDEs are satisfied very accurately (in the pointwise sense for UCG1 as the integrals are Riemann, and not strictly in the pointwise sense for UCG2 since the integrals are Lebesgue) when the local approximations for $u, v, p, \tau_{x x}, \tau_{y y}$ and $\tau_{x y}$ are of class $C^{1,1}\left(\bar{\Omega}_{x y}^{e}\right)$.

In the numerical studies we begin with $\partial p / \partial x=-0.1$ for which a converged solution is obtained and then progressively increase it up to $\partial p / \partial x=-0.275$ using a continuation procedure in which converged solutions at lower $\partial p / \partial x$ are used as initial (or starting) solution in the Newton's linear method. For all values of $\partial p / \partial x$, the computed numerical solutions confirm that $u, \partial u / \partial x$, $\tau_{x x}, \tau_{y y}$ and $\tau_{x y}$ versus $y$ are invariant of spatial location $x$ along $A D$ and are in perfect agreement with those obtained in model problem 1 using fully developed flow 1D numerical studies, hence are not repeated for sake of brevity.

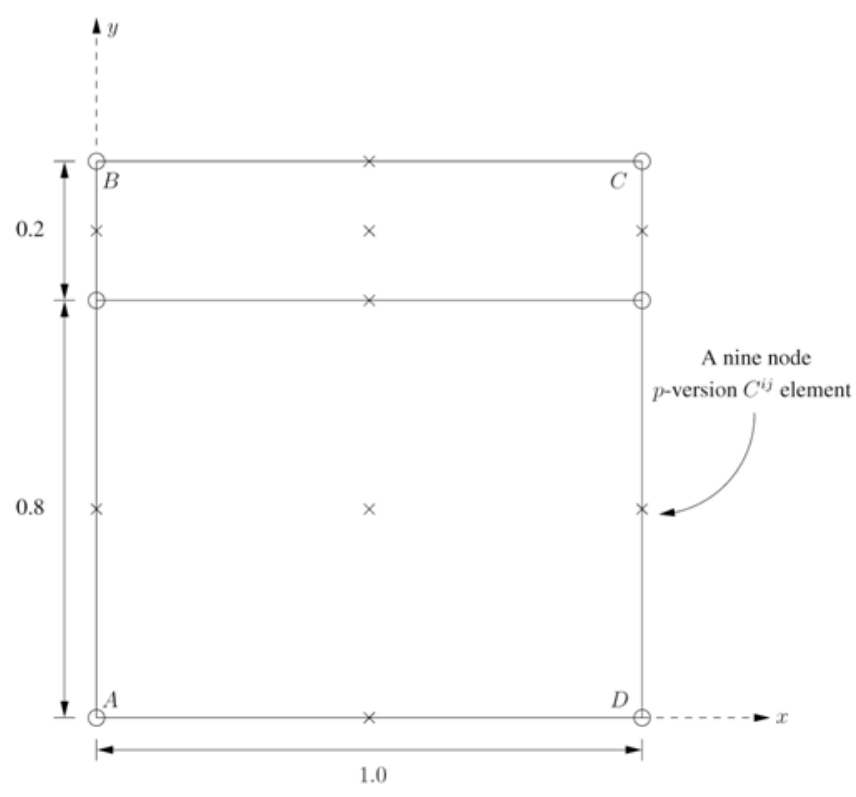

Figure 9: Graded mesh discretization using two 9-node $p$ version elements

\section{SUMMARY AND CONCLUSIONS}

We have presented development of ordered rate constitutive theories for compressible and incompressible thermoviscoelastic fluids in contravariant and covariant bases as well as using Jaumann rates. The theories consider convected time derivatives of up to order ' $m$ ' of the deviatoric Cauchy stress tensor and convected time derivatives of up to order ' $n$ ' of the strain tensor in the chosen basis. The convected time derivative of order ' $m$ ' of the deviatoric Cauchy stress tensor, the heat vector ${ }^{(0)} \overline{\boldsymbol{q}}$ and Helmholtz free energy density $\bar{\Phi}$ are considered as dependent variables in the development of the rate constitutive theories. The argument tensors in the constitutive theories for the deviatoric stress tensor and heat vector are considered to be $\left[\gamma^{(j)}\right] ; j=1,2, \ldots, n$, $\left[{ }_{d} \bar{\sigma}^{(k)}\right] ; k=0,1, \ldots, m-1$, density $\bar{\rho}$, temperature $\bar{\theta}$ and temperature gradient $\overline{\boldsymbol{g}}$ in the contravariant basis. In the case of covariant basis, $\left[\gamma^{(j)}\right]$ and $\left[{ }_{d} \bar{\sigma}^{(k)}\right]$ are replaced by $\left[\gamma_{(j)}\right]$ and $\left[{ }_{d} \bar{\sigma}_{(k)}\right]$ while the other arguments remain the same. When using Jaumann rates, we use $\left[{ }^{(j)} \gamma^{J}\right]$; $j=1,2, \ldots, n$ and $\left[{ }^{(k)}{ }_{d} \bar{\sigma}^{J}\right] ; k=0,1, \ldots, m-1, \bar{\rho}, \bar{\theta}$ and $\overline{\boldsymbol{g}}$ as argument tensors. These rate constitutive theories define ordered thermoviscoelastic fluids of orders $(m, n)$.

Many remarks made in references $[8,9]$ regarding entropy inequality, conditions resulting from it, decomposi- 
tion of the total stress tensor in equilibrium and deviatoric stress tensors, rates of stress and strain tensors in various bases, determination of equilibrium stress for incompressible and compressible cases leading to mechanical and thermodynamic pressure remain the same here as well and hence are not repeated. As in references [8,9], here also, entropy inequality does not provide a mechanism for determining the constitutive equations for the deviatoric stress tensor but only requires that the work expanded due to the deviatoric stress tensor be positive. The development of the rate constitutive theories presented in this paper are based on the theory of generators and invariants. In this approach $\left[{ }_{d} \bar{\sigma}^{(m)}\right]$ and $\overline{\boldsymbol{q}}^{(0)}$ or $\left[{ }_{d} \bar{\sigma}_{(m)}\right]$ and $\overline{\boldsymbol{q}}_{(0)}$ or $\left[{ }^{(m)}{ }_{d} \bar{\sigma}^{J}\right]$ and ${ }^{(0)} \overline{\boldsymbol{q}}^{J}$ are expressed as a linear combination of the combined generators of the argument tensors keeping in mind that $\left[{ }_{d} \bar{\sigma}^{(m)}\right],\left[{ }_{d} \bar{\sigma}_{(m)}\right]$ and $\left[{ }^{(m)}{ }_{d} \bar{\sigma}^{J}\right]$ are symmetric tensors of rank two where as $\overline{\boldsymbol{q}}^{(0)}, \overline{\boldsymbol{q}}_{(0)}$ and ${ }^{(0)} \overline{\boldsymbol{q}}^{J}$ are tensors of rank one. Hence, the combined generators used in the linear combinations for $\left[{ }_{d} \bar{\sigma}^{(m)}\right],\left[{ }_{d} \bar{\sigma}_{(m)}\right]$ or $\left[{ }^{(m)_{d}} \bar{\sigma}^{J}\right]$ must also be symmetric tensors of rank two. Whereas the combined generators used to define $\overline{\boldsymbol{q}}^{(0)}, \overline{\boldsymbol{q}}_{(0)}$ and ${ }^{(0)} \overline{\boldsymbol{q}}^{J}$ must be tensors of rank one. Additionally we must also adhere to minimal basis in these linear combinations. The coefficients in the linear combinations are functions of $\bar{\rho}, \bar{\theta}$ and the combined invariants of the argument tensors of rank one and two and are determined by considering their Taylor series expansions about a known configuration $\underline{\Omega}$ in the combined invariants and $\bar{\theta}$. We make the following specific remarks:

1. The general rate constitutive theories for ordered thermoviscoelastic fluids of orders $(m, n)$ are presented for compressible as well as incompressible thermoviscoelastic fluids. The general theories are specialized for $m=1$ and $n=1$, i.e. thermoviscoelastic fluids of order one in deviatoric Cauchy stress and strain rates. In this case $\left.{ }_{d} \bar{\sigma}^{(1)}\right]$ or $\left[{ }_{d} \bar{\sigma}_{(1)}\right]$ or $\left[{ }^{(1)}{ }_{d} \bar{\sigma}^{J}\right]$ contain $\left[{ }_{d} \bar{\sigma}^{(0)}\right],\left[\gamma^{(1)}\right], \bar{\rho}, \bar{\theta}, \overline{\boldsymbol{g}}$ or $\left[{ }_{d} \bar{\sigma}_{(0)}\right],\left[\gamma_{(1)}\right], \bar{\rho}, \bar{\theta}, \overline{\boldsymbol{g}}$ or $\left[{ }^{(0)}{ }_{d} \bar{\sigma}^{J}\right],\left[{ }^{(1)} \gamma^{J}\right], \bar{\rho}, \bar{\theta}, \overline{\boldsymbol{g}}$ as argument tensors in contravariant and covariant bases as well as using Jaumann rates. The paper also presents the constitutive theory for $\overline{\boldsymbol{q}}^{(0)}, \overline{\boldsymbol{q}}_{(0)}$ and ${ }^{(0)} \overline{\boldsymbol{q}}^{J}$ that contains same argument tensors as $\left[{ }_{d} \bar{\sigma}^{(1)}\right],\left[{ }_{d} \bar{\sigma}_{(1)}\right]$ or $\left[{ }^{(1)} \bar{\sigma}^{J}\right]$. This is essential for consistency of the constitutive theories between the stress tensor and heat vector.

2. The contravariant basis yields upper convected ordered rate constitutive theories. Likewise, covariant basis yields lower convected ordered rate constitutive theories. Use of Jaumann rates yield Jaumann rate constitutive equations. [17] have shown that only contravariant basis is in accordance with the physics of deforming matter when the deformation is finite. As the deformation deviates from the infinitesimal assumption, the rate constitutive equations based on covariant basis and others (such as Jaumann rate equations) become progressively spurious with progressively increasing deformation.

3. It is shown that the Giesekus constitutive model is a subset of ordered thermoviscoelastic fluids (incompressible) of orders $m=1$ and $n=1$. Derivations presented in the paper demonstrate many assumptions needed in the general case of $m=1$, $n=1$ to derive this non-linear viscoelastic constitutive model. The derivation of Giesekus constitutive model presented here is fundamental in understanding the assumptions employed in its derivation which eventually limit its range of applications.

4. The Giesekus constitutive model as used in polymer science has been derived using kinetic theory [2,3] and other theories. The derivation of Maxwell model based on continuum mechanics can be found in [7]. However, the derivation of Giesekus constitutive model based on principles and axioms of continuum mechanics as presented in this paper is the first appearance of this work in the published literature to our knowledge.

5. It is important to note that the Giesekus constitutive model derived here uses the deviatoric Cauchy stress tensor in the development of the rate theories. This is supported by the entropy inequality. The currently used Giesekus constitutive model in published works [2], though similar in the form compared to the model derived here, it uses deviatoric polymer Cauchy stress tensor in the constitutive model with the additional assumptions of (i) decomposition of deviatoric Cauchy stress tensor in solvent and polymer stress tensors (ii) Newton's law of viscosity to define the constitutive theory for the deviatoric solvent Cauchy stress tensor. These are not supported by the principles and axioms of continuum mechanics and in particular of the constitutive theory and the derivation presented in this paper.

6. In polymer science, it is argued $[26,27]$ that decomposition of the deviatoric Cauchy stress in terms of viscous (both solvent and polymer) and elastic components and then expressing viscous stress using Newton's law of viscosity and thus obtaining constitutive equations in terms of deviatoric elastic stress is meritorious (computationally). This approach has two fundamental problems if viewed based on the principles and axioms of continuum mechanics for the constitutive theories. First, the deviatoric Cauchy stress must be a dependent variable in the constitutive theories and not the elastic stress as evident from entropy inequality. 
This argument questions the decomposition. Secondly, use of Newton's law of viscosity must be derivable as opposed to simply using it as a constitutive theory for the viscous stress tensor.

7. All rate theories presented here permit variable material coefficients during the deformation. Even though the Giesekus model can only be derived by neglecting many terms (as shown in the derivations), the dependence of the final material coefficients can be maintained on any (or all) of the desired invariants. This feature permits shear thinning, shear thickening and other behaviors of viscosity etc. to be incorporated in the constitutive models derived here based on experimental and/or empirical relations.

8. Another significant point to note in the present work is that determination of the material coefficients from the coefficients used in the linear combination of the generators to express deviatoric stress or heat vector requires use of Taylor series expansion about the known configuration $\Omega$. This automatically forces the material coefficients to be defined in a known configuration $\underline{\Omega}$ and not in the current configuration. In all presently used works, this is not the case. Variable transport properties as well as dependence of material coefficients on invariants are all expressed using the current configuration. This may be justified when the current and known configurations are in close proximity in terms of deformation field but cannot be supported by the derivation presented in this work.

9. Numerical studies are presented for fully developed flow between parallel plates, and fully developed flow between parallel plates using a two dimensional formulation for a dense polymeric liquid (PIB/C14) using the Giesekus constitutive model derived in this paper as well as currently used Giesekus constitutive model. We use contravariant Cauchy stress tensor and Almansi strain tensors as conjugate measures of the stress and strain tensors in Eulerian description. This yields upper convected Giesekus constitutive models. Numerical results are presented using the upper convected Giesekus constitutive model derived in this paper (UCG1) as well as using the currently used constitutive model in deviatoric polymer stress (UCG2).

10. We choose a value of $\partial p / \partial x$ that is the same in both constitutive models and compute results. For very low values of $\partial p / \partial x$ the velocity fields in the two cases are not drastically different from each other but as $\partial p / \partial x$ increases, both models produced significantly different velocity fields and hence different flow rates. Computed results from fully developed flow between parallel plates using studies in $\mathbb{R}^{1}$, and fully developed flow between parallel plates using two dimensional formulation, i.e. $\mathbb{R}^{2}$ are in perfect agreement when the same constitutive model is used.

\section{ACKNOWLEDGEMENTS}

This research was supported by grant from ARO, Mathematical sciences division under the grant number W-911NF-11-1-0471(FED0061541) to the University of Kansas, Lawrence, Kansas and Texas A \& M University, College Station, Texas. The authors are grateful to Dr. Joseph D. Myers, Program Manager, Scientific Computing, ARO.

\section{REFERENCES}

[1] Maxwell JC. On the dynamical theory of gases. Philos Trans R Soc Lond A 1867; A157: p. 49-88.

[2] Bird RB, Armstrong RC, Hassager O. Dynamics of polymeric liquids, Volume 1: fluid mechanics. 2nd ed. New York: Wiley 1987.

[3] Bird RB, Armstrong RC, Hassager O. Dynamics of polymeric liquids, Volume 2: kinetic theory. 2nd ed. New York: Wiley 1987.

[4] Oldroyd JG. On the formulation of rheological equations of state. Proc R Soc Lond A Math Phys Sci 1950; A200: p. 523-541.

[5] Giesekus H. A simple constitutive equation for polymer fluids based on the concept of deformationdependent tensorial mobility. J Nonnewton Fluid Mech 1982; 11: p. 69-110.

[6] Phan-Thien N, Tanner RI. A nonlinear network viscoelastic model. J Rheol 1978; 22: p. 259-283.

[7] Eringen AC. Mechanics of continua. New York: Wiley 1967.

[8] Surana KS, Nunez D, Reddy JN, Romkes A. Rate constitutive theory for ordered thermofluids. Continuum Mech Thermodyn 2013; 25: p. 625-662.

[9] Surana KS, Nunez D, Reddy JN, Romkes A. Rate constitutive theory for ordered thermoelastic solids. Ann solid struct mech 2012; 3: p. 27-54.

[10] Brown R. A brief account of microscopical observations. Edinburgh J Sci 1828; 5: p. 358-371.

[11] Brown R. Additional remarks on active molecules. Edinburgh J Sci 1829; 1: p. 314-319. 
[12] Udaykumar HS, Tran L, Belk DM, Vanden KJ. An Eulerian method for computation of multimaterial impact with ENO shock-capturing and sharp interfaces. J Comput Phys 2003; 186: p. 136-177.

[13] Tran L, Udaykumar HS. A particle-level set-based sharp interface cartesian grid method for impact, penetration, and void collapse. J Comput Phys 2004; 193: p. 469-510.

[14] Rivlin RS, Ericksen JL. Stress-deformation relations for isotropic materials. Arch Ration Mech Anal 1955; 4: p. 323-425.

[15] Rivlin RS. Further remarks on the stressdeformation relations for isotropic materials. Arch Ration Mech Anal 1955; 4: p. 681-702.

[16] White FM. Fluid mechanics. 7th ed. New York: McGraw-Hill 2010.

[17] Surana KS, MA Y, Reddy JN, Romkes A. The rate constitutive equations and their validity for progressively increasing deformation. Mech Adv Mater Struc 2010; 17: p. 509-533.

[18] Giesekus H. Die Rheologische Zustandsgleichung Elasto-Viskoser Flüssigkeiten - Insbesondere Von Weissenberg-Flüssigkeiten - für Allgemeine und Stationäre Fließvorgänge. J Appl Math Mech 1962; 42: p. 32-61.

[19] Quinzani LM, Armstrong RC, Brown RA. Use of coupled birefringence and LDV studies of flow through a planar contraction to test constitutive equations for concentrated polymer solutions. J Rheol 1995; 39: p. 1201-1227.

[20] Bell B, Surana KS. The $p$-version least squares finite element formulation for two-dimensional, incompressible, non-Newtonian isothermal and non- isothermal fluid flow. Int $\mathrm{J}$ Numer Methods Fluids 1994; 18: p. 127-162.

[21] Bell B, Surana KS. The $p$-version space-time coupled least squares finite element formulation for two-dimensional unsteady incompressible, Newtonian fluid flow. Adv Finite Elem Anal Fluid Dyn 1993; 171: p. 75-87.

[22] Winterscheidt D, Surana KS. p-Version least squares finite element formulation for twodimensional, incompressible fluid flow. Int $\mathrm{J}$ Numer Methods Fluids 1994; 18: p. 43-69.

[23] Surana KS, Allu S, Tenpas PW, Reddy JN. The $k$ version of finite element method in gas dynamics: higher order global differentiability numerical solutions. Int J Numer Methods Eng 2006; 69: p. 11091157.

[24] Surana KS, Reddy JN, Allu S. The $k$-version of finite element method for IVPs: mathematical and computational framework. Int J Comput Methods Eng Sci Mech 2007; 8: p. 123-136.

[25] Surana KS, Allu S, Reddy JN, Tenpas PW. Least squares finite element processes in $h, p, k$ mathematical and computational framework for a nonlinear conservation law. Int J Numer Methods Fluids 2008; 57: p. 1545-1568.

[26] Rajagopalan D, Armstrong RC, Brown RA. Finite element methods for calculation of steady viscoelastic flow using constitutive equations with a Newtonian viscosity. J Nonnewton Fluid Mech 1990; 36: p. 159-192.

[27] Rajagopalan D, Phillips RJ, Armstrong RC, Brown $R A$, Bose $A$. The influence of viscoelasticity on the existence of steady solutions in two-dimensional rimming flow. J Fluid Mech 1992; 235: p. 611-642. 